\title{
825.
}

\section{A MEMOIR ON THE ABELIAN AND THETA FUNCTIONS.}

[Chapters I to III, American Journal of Mathematics, t. v. (1882), pp. 137-179; Chapters IV to VII, ib., t. vII. (1885), pp. 101-167.]

THE present memoir is based upon Clebsch and Gordan's Theorie der Abel'sehen Functionen, Leipzig, 1866 (here cited as C. and G.); the employment of differential rather than of integral equations is a novelty; but the chief addition to the theory consists in the determination which I have made for the cubic curve, and also (but not as yet in a perfect form) for the quartic curve, of the differential expression $d \Pi_{\xi \eta}$ (or as I write it $d \Pi_{12}$ ) in the integral of the third kind $\int_{\alpha}^{\beta} d \Pi_{\xi \eta}$ in the final normal form (endliche Normalform) for which we have (p. 117) $\int_{\xi}^{\eta} d \Pi_{\alpha \beta}=\int_{\alpha}^{\beta} d \Pi_{\xi \eta}$, the limits and parametric points interchangeable. The want of this determination presented itself to me as a lacuna in the theory during the course of lectures on the subject which I had the pleasure of giving at the Johns Hopkins University, Baltimore, U.S.A., in the months January to June, 1882, and I succeeded in effecting it for the cubic curve; but it was not until shortly after my return to England that I was able partially to effect the like determination in the far more difficult case of the quartic curve. The memoir contains, with additional developments, a reproduction of the course of lectures just referred to. I have endeavoured to simplify as much as possible the notations and demonstrations of Clebsch and Gordan's admirable treatise; to bring some of the geometrical results into greater prominence; and to illustrate the theory by examples in regard to the cubic, the nodal quartic, and the general quartic curves respectively. The various chapters are: I, Abel's Theorem; II, Proof of Abel's Theorem; III, The Major Function; IV, The Major Function (continued); V, Miscellaneous Investigations; VI, The Nodal Quartic; VII, The Functions $T, U, V, \Theta$. The paragraphs of the whole memoir will be numbered continuously. 


\section{Chapter I. Abel's Theorem.}

The Differential Pure and Affected Theorems. Art. Nos. 1 to 5.

1. We have a fixed curve and a variable curve, and the differential pure theorem consists in a set of linear relations between the displacements of the intersections of the two curves; in the affected theorem, a linear function of the displacements is equated to another differential expression. I state the two theorems, giving afterwards the necessary explanations.

The pure theorem is

$$
\Sigma(x, y, z)^{n-3} d \omega=0 .
$$

The affected theorem is

$$
\Sigma \frac{(x, y, z)_{12}{ }^{n-2} d \omega}{012}=-\frac{\delta \phi_{1}}{\phi_{1}}+\frac{\delta \phi_{2}}{\phi_{2}} *
$$

2. We have a fixed curve $f=0$, or say the curve $f$, or simply the fixed curve, of the order $n$, with $\delta$.dps, and therefore of the deficiency $\frac{1}{2}(n-1)(n-2)-\delta,=p$. The expression "the dps" means always the $\delta$ dps of $f$.

And we have a variable curve $\phi=0$, or say the curve $\phi$, or simply the variable curve, of the order $m$, passing through the dps and besides meeting the fixed curve in $m n-2 \delta$ variable points.

Moreover, $d \omega$ is the displacement of the current point 0 , coordinates $(x, y, z)$, on the fixed curve, viz. the equation $f=0$ gives

$$
\begin{aligned}
& \frac{d f}{d x} d x+\frac{d f}{d y} d y+\frac{d f}{d z} d z=0 \\
& \frac{d f}{d x} x+\frac{d f}{d y} y+\frac{d f}{d z} z=0
\end{aligned}
$$

and we thence have

$$
\frac{d f}{d x}: \frac{d f}{d y}: \frac{d f}{d z}=y d z-z d y: z d x-x d z: x d y-y d x
$$

so that we have three equal values each of which is put $=d \omega$, viz. we write

$$
\frac{y d z-z d y}{\frac{d f}{d x}}=\frac{z d x-x d z}{\frac{d f}{d y}}=\frac{x d y-y d x}{\frac{d f}{d z}},=d \omega,
$$

and $d \omega$ as thus defined is the displacement.

* For comparison with C. and G. observe that in the equation, p. $47, \quad V=\log \frac{\psi(\eta) \phi(\xi)}{\phi(\eta) \psi(\xi)},=\log \frac{\psi_{2} \phi_{1}}{\phi_{2} \psi_{1}}$ suppose, their $\psi$ belongs to the upper limit and corresponds to my $\phi$ : the equation gives therefore $d V=-\frac{\delta \psi_{1}}{\psi_{1}}+\frac{\delta \psi_{2}}{\psi_{2}}$, agreeing with the formula in the text. 
$(x, y, z)^{n-3}=0$ is the minor curve, viz. the general curve of the order $n-3$, which passes through the dps*; and the function $(x, y, z)^{n-3}$ is the minor function.

1 and 2 are fixed points on $f$, called the parametric points, coordinates $\left(x_{1}, y_{1}, z_{1}\right)$ and $\left(x_{2}, y_{2}, z_{2}\right)$ respectively; and 012 denotes the determinant

$$
\left|\begin{array}{lll}
x, & y, & z \\
x_{1}, & y_{1}, & z_{1} \\
x_{2}, & y_{2}, & z_{2}
\end{array}\right|
$$

so that $012=0$ is the equation of the line joining the points 1 and 2 : this line meets the fixed curve in $n-2$ other points, called the residues of 1,2 .

$(x, y, z)_{12}{ }^{n-2}=0$ is the major curve quoad the points 1 and 2 ; viz. this is the general curve of the order $n-2$, passing through the dps and also through the residues of 1,2 .

But further, the function $(x, y, z)_{12}{ }^{n-2}$ is the proper major function; viz. the implicit factor of the function is so determined that, taking $0=1$, the current point at 1 , that is, writing $\left(x_{1}, y_{1}, z_{1}\right)$ for $(x, y, z)$, the function $(x, y, z)_{12}{ }^{n-2}$ reduces itself to the polar function $\left(x_{2} \frac{d}{d x_{1}}+y_{2} \frac{d}{d y_{1}}+z_{2} \frac{d}{d z_{1}}\right) f_{1}$, afterwards written $n .1^{n-1} 2$, of $f$ : this implies that taking $0=2$, the current point at 2 , the function reduces itself to the polar function $n \cdot 12^{n-1}$.

$\phi_{1}$ is what $\phi$ becomes on writing therein $\left(x_{1}, y_{1}, z_{1}\right)$ for $(x, y, z)$ : and similarly $\phi_{2}$ is what $\phi$ becomes on writing therein $\left(x_{2}, y_{2}, z_{2}\right)$ for $(x, y, z)$.

$\delta$ denotes differentiation in regard only to the coefficients of $\phi$; viz. writing $\phi=(\mathrm{a}, \ldots \chi \chi x, y, z)^{m}$ we have $\delta \phi=(d \mathrm{a}, \ldots \chi \chi x, y, z)^{m}$, and similarly $\delta \phi_{1}$ and $\delta \phi_{2}=\left(d a, \ldots \chi\left(x_{1}, y_{1}, z_{1}\right)^{m}\right.$ and $(d a, \ldots)\left(x_{2}, y_{2}, z_{2}\right)^{m}$ respectively.

The sum $\Sigma$ extends to all the variable intersections of the two curves.

3. As to the meaning of the theorems, consider first the pure theorem. The variable intersections are not all of them arbitrary points on the fixed curve: a certain number of them taken at pleasure on the fixed curve will determine the remaining variable intersections; and there are thus a certain number of integral relations between the coordinates of the variable intersections; to each such integral relation there corresponds a linear relation between the displacements $d \omega$ of these points, or say a displacement-relation. It is precisely these displacement-relations which are given by the theorem, viz. the equation

$$
\Sigma(x, y, z)^{n-3} d \omega=0
$$

breaks up into as many linear relations as there are arbitrary constants in the function $(x, y, z)^{n-3}$, which equated to zero gives a curve of the order $n-3$ passing through the dps; for instance $n=3, \delta=0$, the equation gives the single relation $\Sigma d \omega=0$; but $n=4$, $\delta=0$, the equation gives the three relations $\Sigma x d \omega=0, \Sigma y d \omega=0, \Sigma z d \omega=0$.

* This definition implies that the number of dps is at inost $=\frac{1}{2}(n-1)(n-2)-1$, that is, that the fixed curve is not unicursal. But see post, No. 21. 
4. It is of course important to show, and it will be shown, that the number of independent displacement-relations given by the theorem is equal to the number of independent integral relations between the variable intersections.

5. Observe that the pure theorem gives all the displacement-relations between the variable intersections; we are hereby led to see the nature of the affected theorem. Taking at pleasure on the fixed curve the sufficient number of variable intersections, the coefficients of $\phi$ are thereby determined in terms of the coordinates of the assumed variable intersections*, and hence the value of $-\frac{\delta \phi_{1}}{\phi_{1}}+\frac{\delta \phi_{2}}{\phi_{2}}$ is given as a linear function of the corresponding displacements $d \omega$; and, substituting this value, the affected theorem gives a linear relation between the displacements $d \omega$ of the several variable intersections. But any such linear relation must clearly be a mere linear combination of the displacement-relations $\Sigma(x, y, z)^{n-3} d \omega=0$ given by the pure theorem.

\section{Examples of the Pure Theorem-The Fixed Curve a Cubic. Art. Nos. 6 to 12.}

6. The pure theorem is not applicable to the case $n=2$, the fixed curve a conic: it in fact gives no displacement-relation; and this is as it should be, for the variable intersections are all of them arbitrary.

The next case is $n=3, \delta=0$, the fixed curve a cubic. For greater simplicity the equation is taken in Cartesian coordinates. In general for such an equation, writing in the homogeneous formulæ $z=1$, we have

$$
d \omega=\frac{\frac{d x}{d f}}{d y}=-\frac{d y}{\frac{d f}{d x}},
$$

the two values being of course equal in virtue of $\frac{d f}{d x} d x+\frac{d f}{d y} d y=0$; taking the former value and considering $\frac{d f}{d y}$ as expressed in terms of $x$, let this be called $P$ (of course, $P$ is an irrational function of $x$ ): then we have $d \omega=\frac{d x}{P}$; and similarly $d \omega_{1}=\frac{d x_{1}}{P_{1}}, \& c$.

The fixed curve being then a cubic, let the variable curve be a line; this meets the cubic in three points, say 1,2,3; and any two of these determine the line, and therefore the third point; there should therefore be one integral relation, and consequently one displacement-relation; and this is what is given by the theorem, viz. we have $\Sigma d \omega=0$, that is, $d \omega_{1}+d \omega_{2}+d \omega_{3}=0$, or, what is the same thing,

$$
\frac{d x_{1}}{P_{1}}+\frac{d x_{2}}{P_{2}}+\frac{d x_{3}}{P_{3}}=0
$$

* The coefficients are determined, except it may be as to some constants which remain arbitrary but which disappear from the difference $-\frac{\delta \phi_{1}}{\phi_{1}}+\frac{\delta \phi_{2}}{\phi_{2}}$; this will be explained further on in the text. 
The corresponding integral equation is the equation which expresses that the points $1,2,3$ are in a line, viz. considering $y_{1}, y_{2}, y_{3}$ as given functions of $x_{1}, x_{2}, x_{3}$ respectively, this is

$$
\left|\begin{array}{lll}
x_{1}, & y_{1}, & 1 \\
x_{2}, & y_{2}, & 1 \\
x_{3}, & y_{3}, & 1
\end{array}\right|=0
$$

or, in the notation already made use of for such a determinant, $123=0$.

7. This equation $d \omega_{1}+d \omega_{2}+d \omega_{3}=0$, where $d \omega$ denotes $\frac{d x}{P}$, has a peculiar interpretation when we consider the coefficients of the cubic as arbitrary constants, and therefore the cubic as a curve depending upon nine arbitrary constants*. In taking 1 a point on the curve, we in effect determine $y_{1}$ as a function of $x_{1}$ and the nine constants; and similarly in taking 2 a point on the curve, we determine $y_{2}$ as a function of $x_{2}$ and the nine constants; the points 1 and 2 determine the third intersection 3, and we have thus $x_{3}$ determined as a function of $x_{1}, x_{2}$ and the nine constants.

Considering $x_{3}$ as thus expressed, we have $d x_{3}=\frac{d x_{3}}{d x_{1}} d x_{1}+\frac{d x_{3}}{d x_{2}} d x_{2}$, an equation which must agree with $d \omega_{1}+d \omega_{2}+d \omega_{3}=0$, that is, with $d x_{3}=-\frac{P_{3}}{P_{1}} d x_{1}-\frac{P_{3}}{P_{2}} d x_{2}$. It follows that we have $\frac{d x_{3}}{d x_{1}} \div \frac{d x_{3}}{d x_{2}}=\frac{P_{2}}{P_{1}}$, and taking the logarithms and differentiating with regard to $x_{1}$ and $x_{2}$, we find $\frac{d}{d x_{1}} \frac{d}{d x_{2}} \log \left(\frac{d x_{3}}{d x_{1}} \div \frac{d x_{3}}{d x_{2}}\right)=0$, a partial differential equation of the third order, independent of any particular cubic curve, and satisfied by $x_{3}$ considered as a function of $x_{1}, x_{2}$ and the nine constants. Observe that starting from the expression for $x_{3}$, and proceeding to the differential coefficients of the third order, we have ten equations from which the nine constants can be eliminated, that is, we ought to have a partial differential equation of the third order: and conversely that the equation for $x_{3}$, as containing nine arbitrary constants, is a complete solution of the partial differential equation: the complete solution of the partial differential equation in question is thus the equation which expresses that 3 is the remaining intersection of the line through 1 and 2 with a cubic.

8. The partial differential equation has a geometrical interpretation, or is at least very closely connected with a geometrical property. Consider three consecutive positions of the line, meeting the cubic in the points $1,2,3 ; 1^{\prime}, 2^{\prime}, 3^{\prime}$ and $1^{\prime \prime}, 2^{\prime \prime}, 3^{\prime \prime}$ respectively: the three lines constitute a cubic curve: the nine points are thus the intersections of two cubic curves, or say they are an "ennead" of points: and any eight of the points thus determine uniquely the ninth point.

* This theory was communicated by me to Section A of the British Association at the York meeting. See B. A. Report, 1881, pp. 534, 535, [712], "A Partial Differential Equation connected with the Simplest Case of Abel's Theorem."

C. XII. 
9. As a particular example, let the cubic be $x^{3}+y^{3}-1=0$; then $y=\sqrt[3]{1-x^{3}}$, and $d \omega=\frac{d x}{y^{2}},=\frac{d x}{\left(1-x^{3}\right)^{\frac{2}{3}}} *$; and with these values we have as before the differential relation $d \omega_{1}+d \omega_{2}+d \omega_{3}=0$, and the integral relation $123=0$. I give a direct verification. To find $x_{3}, y_{3}$ the coordinates of the third intersection, we may in the equation of the cubic write $x_{3}, y_{3}, 1=\lambda x_{1}+\mu x_{2}, \lambda y_{1}+\mu y_{2}, \lambda+\mu$ respectively, and then writing for shortness $1^{2} 2=x_{1}{ }^{2} x_{2}+y_{1}{ }^{2} y_{2}-1,12^{2}=x_{1} x_{2}{ }^{2}+y_{1} y_{2}{ }^{2}-1$, we obtain for the determination of $\lambda, \mu$ the equation $\lambda \cdot 1^{22}+\mu \cdot 12^{2}=0$.

This being so, from the equation $123=0$ we obtain by differentiation

$$
\Sigma\left\{d x_{1}\left(y_{2}-y_{3}\right)-d y_{1}\left(x_{2}-x_{3}\right)\right\}=0,
$$

the sum consisting of three terms, the second and third of them being obtained from the one written down by the cyclical interchange of the numbers $1,2,3$. But we have $x_{1}^{2} d x_{1}+y_{1}^{2} d y_{1}=0$, and the equation thus is

$$
\Sigma \frac{d x_{1}}{y_{1}^{2}}\left\{y_{1}^{2}\left(y_{2}-y_{3}\right)+x_{1}^{2}\left(x_{2}-x_{3}\right)\right\}=0:
$$

this will reduce itself to $\Sigma \frac{d x_{1}}{y_{1}{ }^{2}}=0$, if only the three coefficients in \{\} are equal, that is, we ought to have

$$
y_{1}{ }^{2}\left(y_{2}-y_{3}\right)+x_{1}{ }^{2}\left(x_{2}-x_{3}\right)=y_{2}{ }^{2}\left(y_{3}-y_{1}\right)+x_{2}{ }^{2}\left(x_{3}-x_{1}\right)=y_{3}{ }^{2}\left(y_{1}-y_{2}\right)+x_{3}{ }^{2}\left(x_{1}-x_{2}\right) .
$$

Comparing for instance the first and second terms, the equation is

$$
-y_{3}\left(y_{1}^{2}+y_{2}^{2}\right)-x_{3}\left(x_{1}^{2}+x_{2}^{2}\right)+\left(x_{1}^{2} x_{2}+y_{1}^{2} y_{2}+x_{1} x_{2}^{2}+y_{1} y_{2}^{2}\right)=0,
$$

or, as this may be written,

$$
-\left(\lambda y_{1}+\mu y_{2}\right)\left(y_{1}{ }^{2}+y_{2}^{2}\right)-\left(\lambda x_{1}+\mu x_{2}\right)\left(x_{1}^{2}+x_{2}^{2}\right)+(\lambda+\mu)\left(x_{1}^{2} x_{2}+y_{1}^{2} y_{2}+x_{1} x_{2}{ }^{2}+y_{1} y_{2}^{2}\right)=0,
$$

where the whole coefficient of $\lambda$ is $-x_{1}{ }^{3}-y_{1}{ }^{3}+x_{1}{ }^{2} x_{2}+y_{1}{ }^{2} y_{2}$, which in fact is $x_{1}{ }^{2} x_{2}+y_{1}{ }^{2} y_{2}-1$, $=1^{22}$; and similarly the whole coefficient of $\mu$ is $12^{2}$; the equation is thus $\lambda \cdot 1^{22}+\mu \cdot 12^{2}=0$, which is right. The first and second coefficients are thus equal, and in like manner the first and third coefficients are equal; we have thus the required result,

$$
\frac{d x_{1}}{y_{1}^{2}}+\frac{d x_{2}}{y_{2}^{2}}+\frac{d x_{3}}{y_{3}^{2}}=0
$$

10. In all that follows, the cubic might be any cubic whatever, but to fix the ideas I take a particular form.

Let the cubic be $y^{2}-X=0, X$ a cubic function $(x, 1)^{3}$, or say even $X=x .1-x .1-k^{2} x$, then $y=\sqrt{X}, d \omega=\frac{d x}{y},=\frac{d x}{\sqrt{X}}$; and with these values we have the differential relation

* Writing $f=x^{3}+y^{3}-1$ we should have $\frac{d f}{d y}=3 y^{2}$, and therefore $d \omega=\frac{d x}{3 y^{2}}$; but the $\frac{1}{3}$ enters as a common factor in all the $d \omega$ 's, and it may clearly be disregarded: the value in the text, $d \omega=\frac{d x}{y^{2}}$ could of course be obtained by writing, as we may do, $f=\frac{1}{3}\left(x^{3}+y^{3}-1\right)$, and so in other cases. 
$d \omega_{1}+d \omega_{2}+d \omega_{3}=0$, and the integral relation $123=0$. This last equation is an integral of the differential equation $d \omega_{1}+d \omega_{2}+d \omega_{3}=0$; as not containing any arbitrary constant, it is a particular integral.

But regard one of the three points, say 3, as a fixed point, that is, let the line pass through the fixed point 3 of the cubic, and besides meet it in the points 1 and 2. We write $d \omega_{3}=0$, and the differential equation thus is $d \omega_{1}+d \omega_{2}=0$, while the integral equation is as before $123=0$; this equation, as containing one arbitrary constant, is the general integral of $d \omega_{1}+d \omega_{2}=0$.

Let the variable curve be a conic; say the intersections with the cubic are 1, 2, 3, 4, 5, 6. Any five of these points determine the conic, and therefore the sixth point; there is thus one integral relation, the equation $123456=0$, which expresses that the six points are in a conic, and there should therefore be one displacementrelation, viz. this is the equation $\Sigma d \omega=0$, that is, $d \omega_{1}+d \omega_{2}+d \omega_{3}+d \omega_{4}+d \omega_{5}+d \omega_{6}=0$.

We have thus $123456=0$, as a particular integral of

$$
d \omega_{1}+d \omega_{2}+d \omega_{3}+d \omega_{4}+d \omega_{5}+d \omega_{6}=0
$$

If, however, we take 6 a fixed point on the cubic, then we have the same equation as the general integral of $d \omega_{1}+d \omega_{2}+d \omega_{3}+d \omega_{4}+d \omega_{5}=0$.

But taking also 5 a fixed point of the cubic we have as an integral of $d \omega_{1}+d \omega_{2}+d \omega_{3}+d \omega_{4}=0$, the foregoing equation $123456=0$, which contains apparently two arbitrary constants; and so if we also fix the point 4 , or the points 4 and 3 , we have for the differential equations $d \omega_{1}+d \omega_{2}+d \omega_{3}=0$ and $d \omega_{1}+d \omega_{2}=0$, integrals with apparently three arbitrary constants and four arbitrary constants respectively.

11. The explanation is contained in the theory of Residuation on a cubic curve. Take the case $d \omega_{1}+d \omega_{2}+d \omega_{3}=0$, with the integral $123456=0$, containing apparently three arbitrary constants, viz. the relation between the variable points $1,2,3$, is here given by a construction depending on the three fixed points $4,5,6$ on the cubic; it is to be shown that two of these points can always be regarded as no-matter-what* points. To see that this is so, take on the cubic any two no-matter-what points $4^{\prime}, 5^{\prime}$ : then according to the theory referred to, we can find on the cubic a determinate point $6^{\prime}$ such that the points $4^{\prime}, 5^{\prime}$ and $6^{\prime}$ establish between the variable points $1,2,3$, the same relation which is established between them by means of the points 4,5 and 6 ; viz. whether in order to determine the point 3 we draw a conic through $1,2,4,5$ and 6 , or a conic through $1,2,4^{\prime}, 5^{\prime}$ and $6^{\prime}$, we obtain as the remaining intersection of the conic with the cubic one and the same point 3 . The construction of $6^{\prime}$ is, through 4,5 and 6 draw a conic meeting the cubic in any three points 1, 2, 3; through these points and $4^{\prime}, 5^{\prime}$ draw a conic, the remaining intersection of this with the cubic will be the required point $6^{\prime}$, and the point $6^{\prime}$ thus obtained will be a

* The epithet explains, I think, itself ; the point may be any point at pleasure, but it is quite immaterial what point, and for this reason it is not counted as an arbitrary point. The most simple instance is that of two constants presenting themselves in a combination such as $c+c^{\prime}$ : either of them may be regarded as a no-matter-what constant. 
determinate point, independent of the particular conic through 4, 5 and 6 used for the construction. Thus 4 and 5 are replaceable by the no-matter-what points $4^{\prime}$ and $5^{\prime}$, or, what is the same thing, two of the points 4,5 and 6 may be regarded as no-matter-what points, and the number of arbitrary constants is thus reduced to one. And so in other cases, all but one of the fixed points may be regarded as no-matterwhat points, and the integral as containing in each case only one arbitrary constant.

But conversely, it being known that the integral of the differential equation contains but one arbitrary constant, we can thence arrive at the theory of residuation.

12. We might go on to the case where the variable curve is a cubic; there are here nine intersections; any eight of these do not determine the variable cubic, but they do determine the ninth intersection; and there is between the nine intersections one integral relation, and corresponding to it one displacement-relation $\Sigma d \omega=0$, that is, $d \omega_{1}+d \omega_{2}+\ldots+d \omega_{9}=0$, given by the pure theorem. But as to this see further on, where it is shown in general that the number of independent integral relations is equal to the number of independent displacement-relations given by the theorem.

\section{Example of the Affected Theorem-Fixed Curve a Circle. Art. Nos. 13 and 14:}

13. The fixed curve is taken to be the circle $x^{2}+y^{2}-1=0$, and the parametric points 1 and 2 to be the points $(1,0)$ and $(0,1)$ on this circle. The variable curve is taken to be a line, say the line $a x+b y-1=0$, meeting the circle in the points 3 and 4 , coordinates $\left(x_{3}, y_{3}\right)$ and $\left(x_{4}, y_{4}\right)$ respectively.

Starting from the formula

$$
\Sigma \frac{(x, y, 1)_{12}^{0} d \omega}{012}=-\frac{\delta \phi_{1}}{\phi_{1}}+\frac{\delta \phi_{2}}{\phi_{2}}
$$

where the summation extends to the points 3 and $4,(x, y, 1)^{0}{ }_{12}$ is here a constant, $=2.12$, that is, $2\left(x_{1} x_{2}+y_{1} y_{2}-1\right)$, which for the points 1,2 in question is $=-2$. We have 012 denoting the determinant

$$
\left|\begin{array}{lll}
x, & y, & 1 \\
1, & 0, & 1 \\
0, & 1, & 1
\end{array}\right|
$$

which is $=-x-y+1$; and $d \omega=\frac{d x}{2 y}$. Also $\frac{\delta \phi_{1}}{\phi_{1}},=\frac{x d a+y d b}{a x+b y-1}$, is $=\frac{d a}{a-1}$, and similarly $\frac{\delta \phi_{2}}{\phi_{2}}$ is $=\frac{d b}{b-1}$. The formula thus is

$$
\sum \frac{d x}{y(x+y-1)}=-\frac{d a}{a-1}+\frac{d b}{b-1} .
$$

The coefficients $a$ and $b$ are determined by means of the points 3 and 4 , that is, they are functions of $x_{3}, x_{4}$; and considering them as thus expressed, then (inasmuch 
as there is no linear relation between the displacements $\frac{d x_{3}}{y_{3}}$ and $\frac{d x_{4}}{y_{4}}$ of the two arbitrary points 3 and 4 on the circle) the equation must become an identity in regard to the terms in $d x_{3}$ and $d x_{4}$ respectively. It only remains to verify that this is so.

14. Writing $P, Q, R=-y_{3}+y_{4}, x_{3}-x_{4}, x_{3} y_{4}-x_{4} y_{3}$; also $L_{3}$ and $L_{4}=x_{3}+y_{3}-1$ and $x_{4}+y_{4}-1$ respectively, we have $a=P \div R, b=Q \div R$, and the equation is found to be

$$
\frac{d x_{3}}{y_{3} L_{3}}+\frac{d x_{4}}{y_{4} L_{4}}=\frac{1}{(Q-R)(R-P)}\{(Q-R) d P+(R-P) d Q+(P-Q) d R\}
$$

where, substituting for $d y_{3}, d y_{4}$ their values in terms of $d x_{3}, d x_{4}$, we have

$$
d P, d Q, d R=\frac{1}{y_{3}} x_{3} d x_{3}-\frac{1}{y_{4}} x_{4} d x_{4}, \quad \frac{1}{y_{3}} y_{3} d x_{3}-\frac{1}{y_{4}} y_{4} d x_{4}, \quad \frac{1}{y_{3}}\left(x_{3} x_{4}+y_{3} y_{4}\right) d x_{3}-\frac{1}{y_{4}}\left(x_{3} x_{4}+y_{3} y_{4}\right) d x_{4},
$$

and with these values, and by aid of the relations

$$
Q-R, \quad R-P, \quad P-Q=x_{4} L_{3}-x_{3} L_{4}, \quad y_{4} L_{3}-y_{3} L_{4}, \quad-L_{3}+L_{4},
$$

the equation is found to be

$$
\frac{d x_{3}}{y_{3} L_{3}}+\frac{d x_{4}}{y_{4} L_{4}}=\frac{L_{3} L_{4}\left(x_{3} x_{4}+y_{3} y_{4}-1\right)}{\left(x_{4} L_{3}-x_{3} L_{4}\right)\left(y_{4} L_{3}-y_{3} L_{4}\right)}\left(\frac{d x_{3}}{y_{3} L_{3}}+\frac{d x_{4}}{y_{4} L_{4}}\right)
$$

viz. this will be true if only

that is,

$$
L_{3} L_{4}\left(x_{3} x_{4}+y_{3} y_{4}-1\right)-\left(x_{4} L_{3}-x_{3} L_{4}\right)\left(y_{4} L_{3}-y_{3} L_{4}\right)=0 \text {, }
$$

$$
-x_{4} y_{4} L_{3}{ }^{2}-x_{3} y_{3} L_{4}{ }^{2}+L_{3} L_{4}\left(x_{3} x_{4}+y_{3} y_{4}+x_{3} y_{4}+x_{4} y_{3}-1\right)=0 .
$$

But from the values of $L_{3}, L_{4}$ we have $x_{4} y_{4}=\frac{1}{2} L_{4}{ }^{2}+L_{4}, x_{3} y_{3}=\frac{1}{2} L_{3}{ }^{2}+L_{3}$, and the coefficient of $L_{3} L_{4}$ is $=L_{3} L_{4}+L_{3}+L_{4}$; the equation is thus verified.

The example would perhaps have been more instructive if the points 1 and 2 had been left arbitrary points on the circle, but the working out would have been more difficult.

The Variable Intersections of the Two Curves-Number of Independent Integral Relations.

Art. Nos. 15 to 19.

15. Suppose $n=3, \delta=0(p=1)$, the fixed curve a cubic; and suppose successively $m=1,2,3, \ldots$, the variable curve a line, conic, cubic, \&c.

If $m=1$, then two points on the cubic determine the line, and consequently the remaining intersection with the cubic; hence there is one integral relation.

If $m=2$, then five points on the cubic determine the conic, and consequently the remaining intersection with the cubic; hence there is one integral relation. 
If $m=3$, then eight points on the fixed cubic do not determine the variable cubic, but they do determine the ninth intersection. For draw through the eight points a no-matter-what cubic $\chi=0$, the general cubic through the eight points is $\chi+\alpha f=0$, and this meets the fixed cubic in the points $\chi=0, f=0$, that is, in the eight points and in one other point independent of the constant $\alpha$ and therefore completely determinate. Hence in this case also there is one integral relation.

So if $m=4$, then eleven points on the cubic do not determine the quartic, but they do determine the remaining intersection. For draw through the eleven points a no-matter-what quartic $\chi=0$, the general quartic through the eleven points is $\chi+(x, y, z)^{1} f=0$, and this meets the cubic in the points $\chi=0, f=0$, that is, in the eleven points and in one other point independent of the constants of the linear function $(x, y ; z)^{1}$, and therefore completely determinate. Hence there is one integral relation.

And so in general, the fixed curve being a cubic, then, whatever be the order of the variable curve, there is always one integral relation.

16. Suppose next $n=4, \delta=0(p=3)$, the fixed curve a general quartic; and as before suppose successively $m=1,2,3, \ldots$, the variable curve a line, conic, cubic, \&c.

If $m=1$, then two points on the quartic determine the line, and therefore the remaining two intersections; the number of integral relations is $=2$.

If $m=2$, then five points on the quartic determine the conic, and therefore the remaining three intersections; the number of integral relations is $=3$, and similarly if $m=3$, the number of integral relations is $=3$.

If $m=4$, then thirteen points on the fixed quartic do not determine the variable quartic, but they do determine the remaining three intersections. For draw through the thirteen points a no-matter-what quartic $\chi=0$; the general quartic through the thirteen points is $\chi+\alpha f=0$, and this meets the fixed quartic in the points $\chi=0$, $f=0$, that is, in the thirteen points and in three other points, independent of $\alpha$ and thus completely determinate, and the number of integral relations is $=0$; and so in general for any higher value of $m$, the number is still $=3$.

17. Suppose $n=5, \delta=0(p=6)$, the fixed curve a general quintic; and as before suppose $m=1,2,3, \ldots$ successively.

If $m=1$, then two points on the quintic determine the line, and therefore the remaining three intersections; the number of integral relations is $=3$.

If $m=2$, then five points on the quintic determine the conic, and therefore the remaining five intersections; the number of integral relations is $=5$.

If $m=3$, then 9 points on the quintic determine the cubic, and therefore the remaining six intersections; the number of integral relations is $=6$, and so if $m=4$, or if $m=5$ or any greater number, in the first case directly, and in the other cases by consideration of the quintic $\chi+\alpha f=0$, \&c., we find that the number of integral relations is always $=6$. 
18. The reasoning is scarcely altered in the case where the fixed curve has dps; thus considering the general case of a fixed curve $n, \delta, p$ :

If $m=1$, then $2-\delta$ points on the fixed curve (this implies $\delta \ngtr 2$ ) determine the line, and therefore the remaining $n-2 \delta-(2-\delta),=n-2-\delta$ intersections; the number of integral relations is $=n-2-\delta$.

If $m=2$, then $5-\delta$ points on the fixed curve (this implies $\delta \ngtr 5$ ) determine the conic, and therefore the remaining $2 n-2 \delta-(5-\delta),=2 n-5-\delta$ intersections; the number of integral relations is $=2 n-5-\delta$, and so for any value of $m \ngtr n-3$, and indeed for the values $n-2$ and $n-1$; here $\frac{1}{2} m(m+3)-\delta$ points on the fixed curve (this implies $\delta \ngtr \frac{1}{2} m(m+3)$ ) determine the variable curve, and therefore the remaining $m n-2 \delta-\left\{\frac{1}{2} m(m+3)-\delta\right\},=m n-\frac{1}{2} m(m+3)-\delta$ intersections. Hence the number of integral relations is $=m n-\frac{1}{2} m(m+3)-\delta$, that is, $=p-\frac{1}{2}(n-m-1)(n-m-2)$. And thus in the cases $m=n-2$ and $n-1$ the number is $=p$.

If $m=n$, then $\frac{1}{2} n(n+3)-1-\delta$ points on the fixed curve do not determine the variable curve, but they do determine the remaining $n^{2}-2 \delta-\left\{\frac{1}{2} n(n+3)-1-\delta\right\}$, $=\frac{1}{2}(n-1)(n-2)-\delta$, that is, $p$ intersections, and the number of integral relations is thus $=p$; and so, for any higher value of $m$, the number is still $=p$.

19. The conclusion thus is

$$
\begin{aligned}
m \ngtr n-3, \text { the number of integral relations } & =p-\frac{1}{2}(n-m-1)(n-m-2), \\
m=\text { or }>n-2, \quad " \quad, \quad & =p .
\end{aligned}
$$

The integral equations spoken of throughout are of course independent relations.

\section{The Variable Intersections of the Two Curves. Number of Independent Displacement} Relations given by the Pure Theorem. Art. No. 20.

20. The number of displacement-relations given by the pure theorem is = number of constants in minor function $(x, y, z)^{n-3}$, which equated to zero represents a curve through the dps, viz. this is always

$$
\frac{1}{2}(n-1)(n-2)-\delta,=p .
$$

But for $m>n-2$, these relations are not independent. For instance, for $n=4, \delta=0$, $m=1$, the displacement-relations are

$$
\Sigma(x, y, z)^{1} d \omega=0 \text {, that is, } \Sigma x d \omega=0, \quad \Sigma y d \omega=0, \quad \Sigma z d \omega=0,
$$

and conversely from these last equations we have $\Sigma(x, y, z)^{1} d \omega=0$. But in this case the variable curve is a line $a x+b y+c z=0$; hence writing $(x, y, z)^{1}=a x+b y+c z$, the equation $(x, y, z)^{1}=0$ is satisfied for each of the intersections of the line with the quartic, and the corresponding equation $\Sigma(x, y, z)^{1} d \omega=0$ is an identity. Hence the number of independent displacement-relations is $3-1,=2$.

So for $n=5, \delta=0, m=1$, the displacement-relations are

$$
\Sigma(x, y, z)^{2} d \omega=0 \text {, that is, } \Sigma\left(x^{2}, y^{2}, z^{2}, y z, z x, x y\right) d \omega=0 \text {, }
$$


and these six equations give conversely $\Sigma(x, y, z)^{2} d \omega=0$, and in particular they give $\Sigma x(x, y, z)^{1} d \omega=0, \Sigma y(x, y, z)^{1} d \omega=0, \Sigma z(x, y, z)^{1} d \omega=0$. But if $(x, y, z)^{1}$ denote $a x+b y+c z$, then as before we have $(x, y, z)^{1}=0$, for each of the intersections of the two curves, and the last-mentioned three equations are identities. The number of independent displacement-relations is thus $6-3,=3$.

So for $n=5, \delta=0, m=2$. Here if the variable curve is $\phi=(a, \ldots)(x, y, z)^{2}=0$, then taking $(x, y, z)^{2}=(a, \ldots \chi x, y, z)^{2}$, the equation $(x, y, z)^{2}=0$ is satisfied for each of the intersections of the two curves, and the corresponding equation $\Sigma(x, y, z)^{2} d \omega=0$ is an identity; the number of independent displacement-relations is $6-1,=5$.

The reasoning is the same when $\delta$ is not $=0$, and we see generally that for $m<n-2$, or say

$m \ngtr n-3$, the number of independent displacement-relations

while for

$$
=p-\frac{1}{2}(n-m-1)(n-m-2) ;
$$

$$
m=\text { or }>n-2 \text {, the number is }=p ;
$$

since in this case the relations given by the theorem are all of them independent. It thus appears $\dot{a}$ posteriori, that in every case the number of independent displacement-relations given by the pure theorem is equal to the number of independent integral relations.

\section{As to the dps of the Fixed Curve. Art. No. 21.}

21. I conclude with a general remark applicable to the whole of the three chapters. There is no necessity to attend to all or indeed to any of the dps of the fixed curve. Suppose that the fixed curve has $\delta+\delta^{\prime} \mathrm{dps}$, where $\delta$, $\delta^{\prime}$ may be either of them or each $=0$, but attend only to the $\delta \mathrm{dps}$, the $\delta^{\prime} \mathrm{dps}$ being wholly disregarded, and accordingly let the expression the dps mean as before the $\delta$ dps of the fixed curve. No alteration at all is required: only, if $\delta^{\prime}$ be not $=0$, then $p=\frac{1}{2}(n-1)(n-2)-\delta$ will no longer be the deficiency. To obtain the best theorems we use all the $\delta+\delta^{\prime}$ dps: but disregarding the $\delta^{\prime} \mathrm{dps}$, we obtain theorems, as for a curve with $\delta$ dps, which are true, and may frequently be useful either in their original form or with simplifications introduced therein by afterwards taking account of the $\delta^{\prime}$ dps.

\section{Chapter II. Proof of Abel's Theorem.}

Preparation. Art. Nos. 22 and 23.

22. Starting from the equation $\phi=(a, \ldots \chi x, y, z)^{m}=0$ of the variable curve, we have

$$
\begin{aligned}
& \frac{d \phi}{d x} d x+\frac{d \phi}{d y} d y+\frac{d \phi}{d z} d z+\delta \phi=0 \\
& \frac{d \phi}{d x} x+\frac{d \phi}{d y} y+\frac{d \phi}{d z} z
\end{aligned}
$$


where $\delta \phi=\left(d \mathrm{a}, \ldots \chi(x, y, z)^{m}\right.$. Let $\tau$ denote an arbitrary linear function, $=a x+b y+c z$; multiply the two equations by $a x+b y+c z,=\tau$, and $-(a d x+b d y+c d z),=-d \tau$ respectively, and add: we obtain

$$
\begin{aligned}
(y d z-z d y)\left(b \frac{d \phi}{d z}-c \frac{d \phi}{d y}\right)+( & z d x-x d z)\left(c \frac{d \phi}{d x}-a \frac{d \phi}{d z}\right) \\
& +(x d y-y d x)\left(a \frac{d \phi}{d y}-b \frac{d \phi}{d x}\right)+\tau \delta \phi=0
\end{aligned}
$$

introducing $d \omega$, this becomes

$$
d \omega\left[\frac{d f}{d x}\left(b \frac{d \phi}{d z}-c \frac{d \phi}{d y}\right)+\frac{d f}{d y}\left(c \frac{d \phi}{d x}-a \frac{d \phi}{d z}\right)+\frac{d f}{d z}\left(a \frac{d \phi}{d y}-b \frac{d \phi}{d x}\right)\right]+\tau \delta \phi=0,
$$

or observing that $a, b, c$ are the differential coefficients $\frac{d \tau}{d x}, \frac{d \tau}{d y}, \frac{d \tau}{d z}$, the term in [ ] is $J(f, \tau, \phi)$, or say $J(\phi, f, \tau)$, and the equation is

$$
d \omega J(\phi, f, \tau)+\tau \delta \phi=0,
$$

where $J(\phi, f, \tau)$ is the Jacobian, or functional determinant

$$
\begin{aligned}
& \frac{d \phi}{d x}, \frac{d \phi}{d y}, \frac{d \phi}{d z} \\
& \frac{d f}{d x}, \frac{d f}{d y}, \frac{d f}{d z} \\
& \frac{d \tau}{d x}, \frac{d \tau}{d y}, \frac{d \tau}{d z}
\end{aligned} \mid=\frac{d(\phi, f, \tau)}{d(x, y, z)}
$$

and we hence have

$$
d \omega=\frac{-\tau \delta \phi}{J(\phi, f, \tau)}
$$

23. The two theorems thus become

$$
\begin{aligned}
& \Sigma(x, y, z)^{n-3} \frac{\tau \delta \phi}{J(\phi, f ; \tau)}=0 \\
& \Sigma \frac{(x, y, z)_{12}{ }^{n-2}}{012} \frac{-\tau \delta \phi}{J(\phi, f, \tau)}=-\frac{\delta \phi_{1}}{\phi_{1}}+\frac{\delta \phi_{2}}{\phi_{2}}
\end{aligned}
$$

But further, if in the first equation we write $\tau=z$, and in the second equation we retain $\tau$, using it to denote the linear function 012 , the equations become

$$
\begin{aligned}
& \Sigma(x, y, z)^{n-3} \frac{z \delta \phi}{J(\phi, f)}=0 \\
& \Sigma(x, y, z)_{12}{ }^{n-2} \frac{-\delta \phi}{J(\phi, f, \tau)}=-\frac{\delta \phi_{1}}{\phi_{1}}+\frac{\delta \phi_{2}}{\phi_{2}}
\end{aligned}
$$

C. XII. 
where, in the first equation, $J(\phi, f)$ denotes the Jacobian

$$
\begin{array}{ll}
\frac{d \phi}{d x}, & \frac{d \phi}{d y} \\
\frac{d f}{d x}, & \frac{d f}{d y}
\end{array} \mid,=\frac{d(\phi, f)}{d(x, y)} .
$$

In these equations the only differential symbol is the $\delta$ affecting the coefficients $\mathrm{a}, \mathrm{b}, \ldots$ of $\phi, \phi_{1}, \phi_{2}$; the equations are, in respect to the coordinates $(x, y, z)$ of the several variable intersections of the two curves, purely algebraical equations, which are in fact given by Jacobi's Fraction-theorem about to be explained. But for the further reduction of the affected theorem I interpose the next three articles.

The Coordinates $(\rho, \sigma, \tau)$. Art. Nos. 24 to 26 .

24. The letter $\tau$ has just been used to denote the determinant 012: there is often occasion to consider three points $1,2,3$ coordinates $\left(x_{1}, y_{1}, z_{1}\right),\left(x_{2}, y_{2}, z_{2}\right),\left(x_{3}, y_{3}, z_{3}\right)$ respectively; and then writing $\rho, \sigma, \tau$ to denote the determinants $023,031,012$ respectively, and $\Delta$ the determinant 123 , we have identically

$$
\begin{aligned}
& \Delta x=x_{1} \rho+x_{2} \sigma+x_{3} \tau, \\
& \Delta y=y_{1} \rho+y_{2} \sigma+y_{3} \tau, \\
& \Delta z=z_{1} \rho+z_{2} \sigma+z_{3} \tau,
\end{aligned}
$$

which equations, regarding therein the point 0 , coordinates $(x, y, z)$, as a current point, are in fact equations for transformation from the coordinates $(x, y, z)$ to the coordinates $\rho, \sigma, \tau$ belonging to the triangle of reference 123. The points 1 and 2 have been already taken to be on the fixed curve, and it will be assumed that 3 is also a point on this curve.

25. If the function $f$, which equated to zero gives the equation of the fixed curve, be transformed to the new coordinates $(\rho, \sigma, \tau)$, the coefficients of the transformed function are polar functions, each divided by $\nabla^{n}$, viz. the coefficient of $\rho^{n}$ is $\frac{1}{\nabla^{n}} 1^{n}$, which by reason that 1 is a point on the curve is $=0$ (and similarly the coefficients of $\sigma^{n}$ and of $\tau^{n}$ are each $=0$ ), the coefficient of $\rho^{n-1} \sigma$ is $=\frac{1}{\nabla^{n}} n .1^{n-1} 2$; that of $\rho^{n-1} \tau$ is $=\frac{1}{\nabla^{n}} n \cdot 1^{n-1} 3$; that of $\rho^{n-2} \sigma^{2}$ is $=\frac{1}{\nabla^{n}} \frac{1}{2} n(n-1) 1^{n-2} 2^{2}$; and so in other cases. I write this in the form

$$
f=\frac{1}{\nabla^{n}}\left(1^{n}=0, \ldots \chi(\rho, \sigma, \tau)^{n} ;\right.
$$

or we might also use the symbolic form

$$
f=\frac{1}{\Delta^{n}}(\rho 1+\sigma 2+\tau 3)^{n} .
$$


The terms independent of $\tau$ contain, it is clear, the factor $\rho \sigma$, and separating these terms from the others, the equation may be written

$$
f=\frac{1}{\nabla^{n}} \rho \sigma\left(n .1^{n-1} 2, \ldots+\gamma \rho, \sigma\right)^{n-2}+\text { terms involving } \tau .
$$

26. The equations $\tau=0,(\ldots+\gamma \rho, \sigma)^{n-2}=0$, determine the residues of the points 1,2 , and hence the major function $(x, y, z)_{12}{ }^{n-2}$, expressed in terms of $\rho, \sigma, \tau$, and writing therein $\tau=0$, must reduce itself to $(\ldots+\gamma \rho, \sigma)^{n-2}$ multiplied by a constant factor which is at once found to be $=\frac{1}{\nabla^{n-2}}$; for taking the current point at 1 we have $(\rho, \sigma, \tau)=(\Delta, 0,0)$, and the corresponding value of the major function is thus $\frac{1}{\nabla^{n-2}} n \cdot 1^{n-1} 2 \cdot \Delta^{n-2},=n \cdot 1^{n-2} 2$, as it ought to be. We have thus

$$
(x, y, z)_{12}{ }^{n-2}=\frac{1}{\nabla^{n-2}}\left(n .1^{n-1} 2, \ldots+\gamma \rho, \sigma\right)^{n-2}+\text { terms involving } \tau ;
$$

and we hence see that, for $\tau=0$,

an equation which will be useful.

$$
(x, y, z)_{12}{ }^{n-2}=\frac{\Delta^{2} f}{\rho \sigma},
$$

The Preparation for the Affected Theorem resumed. Art. No. 27.

27. In the affected theorem instead of $(x, y, z)$ we introduce the new coordinates $(\rho, \sigma, \tau)$. We have

$$
J(\phi, f, \tau)=\frac{d(\phi, f, \tau)}{d(\rho, \sigma, \tau)} \frac{d(\rho, \sigma, \tau)}{d(x, y, z)}
$$

where the first factor is $=\frac{d(\phi, f)}{d(\rho, \sigma)}$, say that this is $\bar{J}(\phi, f)$, the Jacobian in regard to $\rho, \sigma$ : and the second factor is at once found to be $=\Delta^{2}$. We have consequently

$$
\frac{1}{J(\phi, f, \tau)}=\frac{1}{\Delta^{2} \bar{J}(\phi, f)},
$$

and the equation for the affected theorem becomes

$$
\Sigma(x, y, z)_{12}{ }^{n-2} \frac{\delta \phi}{\bar{J}(\phi, f)}=-\Delta^{2}\left(-\frac{\delta \phi_{1}}{\phi_{1}}+\frac{\delta \phi_{2}}{\phi_{2}}\right),
$$

where $(x, y, z)_{12}{ }^{n-2}$ is to be regarded as standing for its value in terms of $(\rho, \sigma, \tau)$.

\section{Jacobi's Fraction-Theorem. Art. Nos. 28 to 31.}

28. This is the extension of a well-known theorem, which, in a somewhat disguised form, may be thus written: viz. if $U$ be any rational and integral function $(x, 1)^{m}$, then we have

$$
\frac{1}{U}=\Sigma \frac{1}{x-x^{\prime} \cdot J\left(U^{\prime}\right)}
$$


or, introducing an arbitrary constant $A$ by the equation $A U=X$, say this is

$$
\frac{A}{X}\left(=\frac{1}{U}\right)=\Sigma \frac{1}{x-x^{\prime} \cdot J\left(U^{\prime}\right)},
$$

where $U^{\prime}$ is the same function $\left(x^{\prime}, 1\right)^{m}$ of $x^{\prime}$ that $U$ is of $x: J\left(U^{\prime}\right),=\frac{d U^{\prime}}{d x^{\prime}}$ is the Jacobian of $U^{\prime}$, and the summation extends to all roots $x^{\prime}$ of the equation $U^{\prime}=0$ : obviously this is nothing else than the formula for the decomposition of $\frac{1}{U}$ into simple fractions.

29. Take now $U=(x, y, 1)^{m}, V=(x, y, 1)^{n}$, functions of $x, y$ of the degrees $m$ and $n$ respectively, and assume

$$
\begin{aligned}
& A U+B V=X, \text { a function } \quad(x, 1)^{m n}, \\
& C U+D V=Y, \quad \cdots \quad(y, 1)^{m n},
\end{aligned}
$$

viz. let $X=0$ and $Y=0$ be the equations obtained by elimination from $U=0$ and $V=0$ of the $y$ and the $x$ respectively. The forms are

$$
\begin{array}{ll}
A=\left(x, y^{n-1}, 1\right)^{m n-m}, & B=\left(x, y^{m-1}, 1\right)^{m n-n}, \\
C=\left(x^{n-1}, y, 1\right)^{m n-m}, & D=\left(x^{m-1}, y, 1\right)^{m n-n},
\end{array}
$$

where these equations denote the first of them that $A$ is a rational and integral function of the degree $m n-m$ in $x$ and $y$ jointly, but only of the degree $n-1$ in $y$ : and so for the other equations. It follows that

$$
A D-B C=\left(x^{m n-1}, y^{m n-1}, 1\right)^{2 m n-m-n} .
$$

The theorem now is

$$
\frac{A D-B C}{X Y}=\Sigma \frac{1}{x-x^{\prime} \cdot y-y^{\prime} \cdot J\left(U^{\prime}, V^{\prime}\right)},
$$

where $U^{\prime}, V^{\prime}$ are the same functions of $\left(x^{\prime}, y^{\prime}\right)$ that $U, V$ are of $(x, y) ; J\left(U^{\prime}, V^{\prime}\right)$ is the Jacobian $\frac{d\left(U^{\prime}, V^{\prime}\right)}{d\left(x^{\prime}, y^{\prime}\right)}$; and the summation extends to all the simultaneous roots $x^{\prime}, y^{\prime}$ of the equations $U=0, V=0$.

30. For the proof, observe that $A D-B C$ is a sum of terms of the form $x^{\alpha} y^{\beta}$ where $\alpha$ and $\beta$ are each of them at most $=m n-1$; hence $X$ being of the degree $m n$ we have $\frac{x^{a}}{X}=\mathrm{a}$ sum of fractions $\frac{L}{x-x^{\prime}}$, where $x^{\prime}$ is any root of $X=0$; and similarly $\frac{y^{\beta}}{Y}=\mathrm{a}$ sum of fractions $\frac{M}{y-y^{\prime}}$, where $y^{\prime}$ is any root of $Y=0 ;$ multiplying the two expressions and taking the sum for the several terms $\lambda x^{a} y^{\beta}$ of $A D-B C$ we obtain a formula

$$
\frac{A D-B C}{\bar{X} Y}=\Sigma \frac{K}{x-x^{\prime} \cdot y-y^{\prime}}
$$

where the summation extends to all the combinations of the $m n$ values of $x^{\prime}$ with the $m n$ values of $y$. But such a formula existing, the coefficients $K$ may be determined 
in the usual manner, viz. multiplying by $X Y$ and then writing $x=x^{\prime}, y=y^{\prime}$, there is on the right-hand only one term which does not vanish, and we find

$$
(A D-B C)_{x^{\prime} y^{\prime}}=K\left(\frac{X}{x-x^{\prime}}\right)_{x^{\prime}}\left(\frac{Y}{y-y^{\prime}}\right)_{y^{\prime}}=K\left(\frac{d X}{d x} \frac{d Y}{d y}\right)_{x^{\prime} y^{\prime}}
$$

where the factor which multiplies $K$ does not vanish.

We distinguish the cases where $\left(x^{\prime}, y^{\prime}\right)$ are corresponding or non-corresponding roots of $X=0, Y=0$; viz. corresponding roots are those for which $U=0, V=0$, but for non-corresponding roots these equations do not hold good; there are obviously $m n$ pairs of corresponding roots.

In the latter case $(A D-B C) U=D X-B Y ;(A D-B C) V=-C X+A Y$, and since for the values in question $X, Y$ each vanish, but $U, V$ do not each of them vanish, we must for these values have $A D-B C=0$, and the foregoing equation for $K$ gives then $K=0$.

31. The formula thus is

$$
\frac{A D-B C}{X Y}=\Sigma \frac{K}{x-x^{\prime} \cdot y-y^{\prime}}
$$

where the summation now extends only to corresponding roots $x^{\prime}, y^{\prime}$, for which we have $U=0, \quad V=0$. We have for $K$ the foregoing expression, which, to complete the determination, we write under the form

$$
A D-B C=K J(X, Y)_{x^{\prime} y^{\prime}}
$$

this is allowable, for $J(X, Y),=\frac{d(X, Y)}{d(x, y)}$, differs from $\frac{d X}{d x} \frac{d Y}{d y}$ only by the zero term $-\frac{d X}{d y} \frac{d Y}{d x}$. Moreover, differentiating the expressions for $X, Y$, and considering $(x, y)$ as therein standing for a pair of corresponding roots $\left(x^{\prime}, y^{\prime}\right)$, the terms containing $U, V$ will all vanish; we thus in effect differentiate as if $A, B, C, D$ were constants, and the result is $(A D-B C) J(U, V)$, or say this is $(A D-B C)_{x^{\prime} y^{\prime}} J\left(U^{\prime}, V^{\prime}\right)$ : hence, in the equation for $K$, the factor $(A D-B C)_{x^{\prime} y^{\prime}}$ divides out, and we have $1=K J\left(U^{\prime}, V^{\prime}\right)$; hence the required formula is

$$
\frac{A D-B C}{X Y}=\Sigma \frac{1}{x-x^{\prime} \cdot y-y^{\prime}} . J\left(U^{\prime}, V^{\prime}\right)
$$

the summation extending to all the simultaneous roots $\left(x^{\prime}, y^{\prime}\right)$ of $U=0, V=0$.

\section{Homogeneous Form of the Fraction-Theorem. Art. No. 32.}

32. For $x, y, x^{\prime}, y^{\prime}$ we write $\frac{x}{z}, \frac{y}{z}, \frac{x^{\prime}}{z^{\prime}}, \frac{y^{\prime}}{z^{\prime}}$; supposing that $U, V$ now denote homogeneous functions $(x, y, z)^{m},(x, y, z)^{n}$, and that we have

$$
\begin{aligned}
& A U+B V=X,=(x, z)^{m n},=\alpha x^{m n}+\ldots, \\
& C U+D V=Y,=(y, z)^{m n},=\beta y^{m n}+\ldots,
\end{aligned}
$$


where the forms are

$$
\begin{gathered}
A=\left(x, y^{n-1}, z\right)^{m n-m}, \quad B=\left(x, y^{m-1}, z\right)^{m n-n}, \\
C=\left(x^{n-1}, y, z\right)^{m n-m}, \quad D=\left(x^{m-1}, y, z\right)^{m n-n}, \\
A D-B C=\left(x^{m n-1}, y^{m n-1}, z\right)^{2 m n-m-n},
\end{gathered}
$$

viz. the degree of $A$ in $(x, y, z)$ is $=m n-m$, but $y$ rises only to the degree $n-1$, and so in other cases; then the theorem becomes

$$
\frac{z^{m+n-2}(A D-B C)}{X Y}=\Sigma \frac{z^{\prime m+n}}{x z^{\prime}-x^{\prime} z \cdot y z^{\prime}-y^{\prime} z} \cdot J\left(U^{\prime}, V^{\prime}\right)
$$

where $J\left(U^{\prime}, V^{\prime}\right)$ denotes the Jacobian $\frac{d\left(U^{\prime}, V^{\prime}\right)}{d\left(x^{\prime}, y^{\prime}\right)}$, and the summation extends to the simultaneous roots $\left(x^{\prime}, y^{\prime}, z^{\prime}\right)$ of $U=0, V=0$.

It is proper to introduce into the formula $\tau^{\prime}$, an arbitrary linear function $a x^{\prime}+b y^{\prime}+c z^{\prime}$ of $\left(x^{\prime}, y^{\prime}, z^{\prime}\right)$ : observe that, in the Jacobian, $\left(x^{\prime}, y^{\prime}, z^{\prime}\right)$ have always values for which $U^{\prime}=0, V^{\prime}=0$ : we have therefore

and thence

$$
\begin{aligned}
& x^{\prime} \frac{d U^{\prime}}{d x^{\prime}}+y^{\prime} \frac{d U^{\prime}}{d y^{\prime}}+z^{\prime} \frac{d U^{\prime}}{d z^{\prime}}=0 \\
& x^{\prime} \frac{d V^{\prime}}{d x^{\prime}}+y^{\prime} \frac{d V^{\prime}}{d y^{\prime}}+z^{\prime} \frac{d V^{\prime}}{d z^{\prime}}=0
\end{aligned}
$$

$$
x^{\prime}: y^{\prime}: z^{\prime}=\frac{d\left(U^{\prime}, V^{\prime}\right)}{d\left(y^{\prime}, z^{\prime}\right)}: \frac{d\left(U^{\prime}, V^{\prime}\right)}{d\left(z^{\prime}, x^{\prime}\right)}: \frac{d\left(U^{\prime}, V^{\prime}\right)}{d\left(x^{\prime}, y^{\prime}\right)},
$$

and if the expressions on the right-hand are for a moment called $A^{\prime}, B^{\prime}, C^{\prime}$, then writing $\tau^{\prime}=a x^{\prime}+b y^{\prime}+c z^{\prime}$, we have $J\left(U^{\prime}, V^{\prime}, \tau^{\prime}\right)=a A^{\prime}+b B^{\prime}+c C^{\prime},=\frac{\tau^{\prime}}{z^{\prime}} C^{\prime},=\frac{\tau^{\prime}}{z^{\prime}} J\left(U^{\prime}, V^{\prime}\right)$, that is,

or the equation becomes

$$
\frac{1}{J\left(U^{\prime}, V^{\prime}\right)}=\frac{\tau^{\prime}}{z^{\prime} J\left(U^{\prime}, V^{\prime}, \tau^{\prime}\right)}
$$

$$
\frac{z^{m+n-2}(A D-B C)}{X Y}=\Sigma \frac{z^{\prime m+n-1} \tau^{\prime}}{x z^{\prime}-x^{\prime} z \cdot y z^{\prime}-y^{\prime} z} . J\left(U^{\prime}, V^{\prime}, \tau^{\prime}\right)
$$

the summation being as before.

Resulting Special Theorems. Art. Nos. 33 to 35.

33. Reverting to the Cartesian form, we have

$$
\begin{aligned}
\frac{x y(A D-B C)}{X Y} & =\Sigma \frac{1}{J\left(U^{\prime}, V^{\prime}\right)}\left(1+\frac{x^{\prime}}{x}+\ldots\right)\left(1+\frac{y^{\prime}}{y}+\ldots\right), \\
& =\Sigma \frac{1}{J\left(U^{\prime}, V^{\prime}\right)}\left\{1+H_{1}\left(\frac{x^{\prime}}{x}, \frac{y^{\prime}}{y}\right)+H_{2}\left(\frac{x^{\prime}}{x}, \frac{y^{\prime}}{y}\right)+\ldots\right\}
\end{aligned}
$$

where $H_{m}$ is the homogeneous sum of the order $m$,

$$
H_{1}(u, v)=u+v, H_{2}(u, v)=u^{2}+u v+v^{2}, \& c .
$$


The left-hand side is

$$
(A D-B C)\left(\frac{1}{\alpha x^{m n-1}}+\frac{\& c .}{x^{m n}} \ldots\right)\left(\frac{1}{\beta y^{m n-1}}+\frac{\& c .}{y^{m n}}+\ldots\right)
$$

and in $A D-B C$ the terms of the highest order in $(x, y)$, say $(A D-B C)_{0}$ are

$$
(A D-B C)_{0}=(x y)^{m n-m-n+1}(a, b, \ldots, k+\gamma x, y)^{m+n-2} .
$$

There is thus on the left-hand no term which is in $(x, y)$ of a higher degree than $-(m+n-2)$; hence on the right-hand every term of a higher degree than this in $(x, y)$ must vanish, viz. we must have

$$
0=\Sigma \frac{x^{\prime \alpha} y^{\prime} \beta}{J\left(U^{\prime}, V^{\prime}\right)} \text { so long as } \alpha+\beta \ngtr m+n-3,
$$

or, what is the same thing, we must have

$$
0=\Sigma \frac{\left(x^{\prime}, y^{\prime}, 1\right)^{m+n-3}}{J\left(U^{\prime}, V^{\prime}\right)}, \text { say the }(m+n-3) \text { theorem, }
$$

where $\left(x^{\prime}, y^{\prime}, 1\right)^{m+n-3}$ is the arbitrary function of the degree $m+n-3$.

34. Passing to the next lower degree $-(m+n-2)$, we have

$$
\frac{1}{\alpha \beta(x y)^{m+n-2}}\left(a, b, \ldots, k+\gamma(x, y)^{m+n-2}=\Sigma \frac{1}{J\left(U^{\prime}, V^{\prime}\right)} H_{m+n-2}\left(\frac{x^{\prime}}{x}, \frac{y^{\prime}}{y}\right)\right.
$$

and if in $(a, b, \ldots, k+\gamma x, y)^{m+n-2}$ we consider any term $g x^{m+n-2-p} y^{m+n-2-q}$, where $p+q=m+n-2$, then we have on the left-hand the term $\frac{g}{\alpha \beta x^{p} y^{q}}$, and the corresponding term on the right-hand must be

that is, we have

$$
\Sigma \frac{1}{J\left(U^{\prime}, V^{\prime}\right)} \frac{x^{\prime p} y^{q}}{x^{p} y^{q}}
$$

$$
\frac{g}{\alpha \beta}=\Sigma \frac{x^{\prime p} y^{\prime q}}{J\left(U^{\prime}, V^{\prime}\right)} \text {. }
$$

But from the foregoing expression for $(A D-B C)_{0}$, it appears that $(A D-B C)_{0}$ contains the term $g x^{m n-1-p} y^{m n-1-q}$, and it hence appears that $g$ is the constant term of the quotient $(A D-B C)_{0}$ divided by $x^{m n-1-p} y^{m n-1-q}$, or as this may be written

$$
g=\text { const. of } \frac{(A D-B C)_{0} x^{p} y^{q}}{(x y)^{m n-1}}
$$

comparing the two values of $g$, we obtain

$$
\text { const. of } \frac{(A D-B C)_{0} x^{p} y^{q}}{\alpha \beta(x y)^{m n-1}}=\Sigma \frac{x^{\prime p} y^{\prime q}}{J\left(U^{\prime}, V^{\prime}\right)},(p+q=m+n-2),
$$

and we hence derive

$$
\text { Const. of } \frac{(A D-B C)_{0}(x, y)^{m+n-2}}{\alpha \beta(x y)^{m n-1}}=\Sigma \frac{\left(x^{\prime}, y^{\prime}\right)^{m+n-2}}{J\left(U^{\prime}, V^{\prime}\right)},
$$


where $(x, y)^{m+n-2}$ is the general function of the degree $m+n-2$, and, of course, $\left(x^{\prime}, y^{\prime}\right)^{m+n-2}$ is the same function of $x^{\prime}, y^{\prime}$. The two functions may be written $(x, y, 0)^{m+n-2}$ and $\left(x^{\prime}, y^{\prime}, 0\right)^{m+n-2}$; and this being so we may on the right-hand write instead $\left(x^{\prime}, y^{\prime}, 1\right)^{m+n-2}$, for, by so doing we introduce in the numerator of the fraction new terms of an order not exceeding $m+n-3$, and by the $(m+n-3)$ theorem already obtained the sum $\Sigma$ of the quotient of such terms by $J\left(U^{\prime}, V^{\prime}\right)$ is $=0$. We thus have

Const. of $\frac{(A D-B C)_{0}(x, y, 0)^{m+n-2}}{\alpha \beta(x y)^{m n-1}}=\Sigma \frac{\left(x^{\prime}, y^{\prime}, 1\right)^{m+n-2}}{J\left(U^{\prime}, V^{\prime}\right)}$, say the $(m+n-2)$ theorem,

where $\left(x^{\prime}, y^{\prime}, 1\right)^{m+n-2}$ is the general non-homogeneous function of the degree $m+n-2$, and $(x, y, 0)^{m+n-2}$ is obtained from it by attending only to the terms of the highest degree $m+n-2$, and therein substituting $x, y$ for $x^{\prime}, y^{\prime}$.

35. We may, it is clear, in the equations for the $(m+n-3)$ and for the $(m+n-2)$ theorems respectively, omit the accents on the right-hand sides; doing this, and moreover in each equation transposing the two sides, the two special theorems are

$$
\begin{array}{ll}
\Sigma \frac{(x, y, 1)^{m+n-3}}{J(U, V)}=0, & (m+n-3) \text { theorem, } \\
\Sigma \frac{(x, y, 1)^{m+n-2}}{J(U, V)}=\text { const. of } \frac{(A D-B C)_{0}(x, y, 0)^{m+n-2}}{\alpha \beta(x y)^{m n-1}},(m+n-2) \text { theorem, }
\end{array}
$$

\section{Homogeneous Form of the Special Theorems. Art. No. 36.}

36. Writing $\frac{x}{z}, \frac{y}{z}$ for $x, y$, and introducing as before the arbitrary linear function $\tau=a x+b y+c z$, we at once obtain, $U, V$ being now homogeneous functions $(x, y, z)^{m}$ and $(x, y, z)^{n}$ respectively, and the $A, B, C, D$ being also homogeneous functions accordingly,

$$
\begin{array}{ll}
\Sigma \frac{z(x, y, z)^{m+n-3}}{J(U, V)}=0, & (m+n-3) \text { theorem, } \\
\Sigma \frac{\tau(x, y, z)^{m+n-2}}{z J(U, V, \tau)}=\text { const. of } \frac{(A D-B C)_{0}(x, y, 0)^{m+n-2}}{\alpha \beta(x y)^{m n-1}},(m+n-2) \text { theorem, }
\end{array}
$$

where the suffix 0 denotes that we are in $A D-B C$ to write $z=0$.

If in the last formula we change throughout the letters $x, y, z$ into $\rho, \sigma, \tau$ (that is, consider $U, V$ as given functions of $\rho, \sigma, \tau$ ), but retain $\tau$ as standing for the particular function $0 \rho+0 \sigma+1 \tau$, then the formula becomes

$$
\Sigma \frac{(\rho, \sigma, \tau)^{m+n-2}}{\bar{J}(U, V)}=\text { const. of } \frac{(A D-B C)_{0}(\rho, \sigma, \tau)^{m+n-2}}{\alpha \beta(\rho \sigma)^{m n-1}},(m+n-2) \text { theorem, }
$$

where $\bar{J}(U, V)$ denotes $\frac{d(U, V)}{d(\rho, \sigma)}$, the Jacobian in regard to $\rho, \sigma$. 
The effect of $d p s$ of the Curves $U=0, V=0$. Art. Nos. 37 and 38 .

37. We must, in regard to the foregoing special theorems, consider the effect of any dps of the curves $U=0, V=0$.

Suppose one of the curves, say $V$, has a dp, but that the other curve $U$ does not pass through it; the $\mathrm{dp}$ is not an intersection of $U, V$, and the theorems are in nowise affected.

If $U$ passes through the $d p$, then the $d p$ counts twice among the intersections of $U, V$; at the dp we have $J\left(U^{\prime}, V^{\prime}\right)=0$, and (to fix the ideas, attending to the $(m+n-3)$ theorem) the sum $\Sigma \frac{(x, y, z)^{m+n-3}}{J(U, V)}$ will contain two infinite terms; these may very well, and indeed (assuming that the theorem remains true) must have a finite sum, but except by the theorem itself, this finite sum is not calculable, and the theorem thus becomes nugatory.

If, however, the curve $(x, y, z)^{m+n-3}=0$ be a curve passing through the $\mathrm{dp}$, then considering, instead, the case where the last-mentioned curve and $U$ each approach indefinitely near to the dp of $V$; there are two intersections of $U, V$ indefinitely near to each other and to the dp; at either intersection, the numerator $\left(x^{\prime}, y^{\prime}, z^{\prime}\right)^{m+n-3}$ and the denominator $J(U, V)$ are infinitesimals of the same order, say the first, and the fraction has a finite-value; the finite values for the two intersections have not in general a zero sum, and consequently in the limit it would not be allowable to disregard the intersections belonging to the $\mathrm{dp}$.

38. But if the numerator curve $(x, y, z)^{m+n-3}=0$ passes twice through the $\mathrm{dp}$ (that is, has there a dp), then reverting to the two consecutive intersections, at either of these the denominator $J(U, V)$ is as before an infinitesimal of the first order, but the numerator $(x, y, z)^{m+n-3}$ is an infinitesimal of the second order, and in the limit the value of the fraction is $=0$; we may in this case disregard the intersections belonging to the $\mathrm{dp}$; and so in general, the curve $(x, y, z)^{m+n-3}=0$ passing twice through each dp of $U$ which lies upon $V$, we have

$$
\Sigma \frac{z(x, y, z)^{m+n-3}}{J(U, V)}=0,
$$

the summation now extending to all the intersections of $U, V$ other than the dps in question, which are to be disregarded. And the like in regard to the other theorem

$$
\Sigma \frac{(x, y, z)^{m+n-2}}{J(U, V)}=\text { const. of } \frac{(A D-B C)_{0}(x, y, 0)^{m+n-2}}{\alpha \beta(x y)^{m n-1}} .
$$

\section{The Pure Theorem.-Completion of the Proof. Art. No. 39.}

39. The theorem was reduced to

$$
\Sigma \frac{z(x, y, z)^{n-3} \delta \phi}{J(\phi, f)}=0
$$

which is therefore the equation to be proved.

c. XII. 
The $(m+n-3)$ theorem, writing therein $\phi, f$ in place of $U, V$ respectively (the degrees being as before $m$ and $n$ ), is

$$
\Sigma \frac{z(x, y, z)^{m+n-3}}{J(U, V)}=0
$$

Here $(x, y, z)^{m+n-3}$ is an arbitrary function of the degree $m+n-3$, and this may therefore be put $=(x, y, z)^{n-3} \delta \phi$, where $\delta \phi,=(d a, \ldots \chi x, y, z)^{m}$, is a function of the degree $m$; and since the curve $\phi=0$ passes always through the dps of $f$, and varies subject to this condition, the curve $\delta \phi=0$ will also pass through the dps; hence taking $(x, y, z)^{n-3}=0$ a curve through the dps, the curve $(x, y, z)^{n-3} \delta \phi=0$ is a curve passing twice through each of dps, and the $(m+n-3)$ theorem thus gives the equation which was to be proved. This completes the proof of the pure theorem

$$
\Sigma(x, y, z)^{n-3} d \omega=0 .
$$

The Affected Theorem.-Completion of the Proof. Art. Nos. 40 and 41.

40. The theorem was reduced to

$$
\Sigma \frac{(x, y, z)_{12}{ }^{n-2} \delta \phi}{\bar{J}(\phi, f)}=-\Delta^{2}\left(-\frac{\delta \phi_{1}}{\phi_{1}}+\frac{\delta \phi_{2}}{\phi_{2}}\right)
$$

which is therefore the equation to be proved.

The $(m+n-2)$ theorem, written with $(\rho, \sigma, \tau)$ in place of $(x, y, z)$, and putting therein $\phi, f$ for $U, V$, is

$$
\Sigma \frac{(\rho, \sigma, \tau)^{m+n-2}}{\bar{J}(\phi, f)}=\text { const. of } \frac{(A D-B C)_{0}(\rho, \sigma, 0)^{m+n-2}}{\alpha \beta(\rho \sigma)^{m n-1}},
$$

where it will be recollected that the suffix $(0)$ denotes that $\tau$ is to be put $=0$. Here $(\rho, \sigma, \tau)^{m+n-2}$ is an arbitrary function of the degree $m+n-2$, and this may therefore be put $=(x, y, z)_{12}{ }^{n-2} \delta \phi$, the two factors being each of them considered as expressed in terms of $(\rho, \sigma, \tau)$; and since each of the curves $(x, y, z)_{12}{ }^{n-2}=0$ and $\delta \phi=0$ passes through the dps of $f$, the curve $(x, y, z)_{12}{ }^{n-2} \delta \phi=0$ is a curve passing twice through each of the dps. We have therefore

$$
\Sigma \frac{(x, y, z)_{12}{ }^{n-2} \delta \phi}{\bar{J}(\phi, f)}=\text { const. of } \frac{(A D-B C)_{0}(x, y, z)_{12}{ }^{n-2} \delta \phi_{0}}{\alpha \beta(\rho \sigma)^{m n-1}}
$$

where on the right-hand side $(x, y, z)_{12}{ }^{n-2}$ is considered as a function of $\rho, \sigma, \tau$, and we are to put therein $\tau=0$; it has been seen (No. 26) that the value is $=\frac{f_{0} \Delta^{2}}{\rho \sigma}$, where $f_{0}$ is what $f$, considered as a function of $\rho, \sigma, \tau$, becomes on writing therein $\tau=0$; the right-hand side thus becomes

$$
=\text { const. of } \frac{(A D-B C)_{0} f_{0} \Delta^{2} \delta \phi_{0}}{\alpha \beta(\rho \sigma)^{m n}} \text {. }
$$


41. But for $\tau=0$, we have

and hence

$$
\begin{aligned}
& A_{0} \phi_{0}+B_{0} f_{0}=\alpha \rho^{m n}, \\
& C_{0} \phi_{0}+D_{0} f_{0}=\beta \sigma^{m n},
\end{aligned}
$$

$$
(A D-B C)_{0} f_{0}=A_{0} \beta \sigma^{m n}-C_{0} \alpha \rho^{m n},
$$

and the right-hand side thus becomes, say

$$
-\Delta^{2} \text { const. of }\left(-\frac{A_{0}}{\alpha \rho^{m n}}+\frac{C_{0}}{\beta \sigma^{m n}}\right) \delta \phi_{0} .
$$

But in calculating the constant of $\frac{A_{0}}{\alpha \rho^{m n}} \delta \phi_{0}$, we may suppose not only $\tau=0$, but also $\sigma=0$ : we then have $\phi_{0}=(x, y, z)^{m},=\left(\frac{\rho}{\Delta}\right)^{m}\left(x_{1}, y_{1}, z_{1}\right)^{m},=\left(\frac{\rho}{\Delta}\right)^{m} \phi_{1}$, and hence also $\delta \phi_{0}=\left(\frac{\rho}{\Delta}\right)^{m} \delta \phi_{1}$

Similarly, in calculating the constant of $\frac{B_{0}}{\beta \sigma^{m n}} \delta \phi_{0}$, we may suppose not only $\tau=0$, but also $\rho=0$ : we then have $\phi_{0}=(x, y, z)^{m},=\left(\frac{\sigma}{\Delta}\right)^{m}\left(x_{2}, y_{2}, z_{2}\right)^{m},=\left(\frac{\sigma}{\Delta}\right)^{m} \phi_{2}$, and hence $\delta \phi_{0}=\left(\frac{\sigma}{\Delta}\right)^{m} \delta \phi_{2}$

Moreover, in the equations

$$
\begin{aligned}
& A_{0} \phi_{0}+B_{0} f_{0}=\alpha \rho^{m n}, \\
& C_{0} \phi_{0}+D_{0} f_{0}=\beta \sigma^{m n},
\end{aligned}
$$

writing in the first equation $\sigma=0$, we find $A_{0}\left(\frac{\rho}{\Delta}\right)^{m} \phi_{1}=\alpha \rho^{m n}$, that is, $\frac{A_{0}}{\alpha \rho^{m n}}=\left(\frac{\Delta}{\rho}\right)^{m} \frac{1}{\phi_{1}}$; and similarly writing in the second equation $\rho=0$, we find $C_{0}\left(\frac{\sigma}{\Delta}\right)^{m} \phi_{2}=\beta \sigma^{m n}$, that is, $\frac{C_{0}}{\beta \sigma^{m n}}=\left(\frac{\Delta}{\sigma}\right)^{m} \frac{1}{\phi_{2}}:$ and the expression thus becomes

$$
=-\Delta^{2}\left(-\frac{\delta \phi_{1}}{\phi_{1}}+\frac{\delta \phi_{2}}{\phi_{2}}\right)
$$

giving the equation which was to be proved. This completes the proof of the affected theorem

$$
\Sigma \frac{(x, y, z)_{12}{ }^{n-2} d \omega}{012}=-\frac{\delta \phi_{1}}{\phi_{1}}+\frac{\delta \phi_{2}}{\phi_{2}}
$$




\section{Chapter III. The Major Function $(x, y, z)_{12}{ }^{n-2}$.}

Analytical Expression of the Function. Art. Nos. 42 to 49.

42. The function has been defined by the conditions that the curve $(x, y, z)_{12}{ }^{n-2}=0$ shall pass through the dps, and also through the $n-2$ residues of the parametric points 1, 2: and moreover, that on writing therein $\left(x_{1}, y_{1}, z_{1}\right)$ for $(x, y, z)$, the function shall become $=n \cdot 1^{n-1} 2$. Obviously the function is not completely determined: calling it $\Omega$ (or when required $\Omega_{12}$ ), then if $\Omega^{\prime}$ be any particular form of it, the general form is $\Omega=\Omega^{\prime}+(x, y, z)^{n-3}$.012, where $(x, y, z)^{n-3}$ is the general minor function, viz. $(x, y, z)^{n-3}=0$ is a curve passing through the dps: the major function thus contains $\frac{1}{2}(n-1)(n-2)-\delta,=p$, arbitrary constants.

Agreeing with the definition, we have the before-mentioned equation

$$
\Omega=\frac{1}{\Delta^{n-2}}\left(n .1^{n-1} 2, \ldots+\gamma \rho, \sigma\right)^{n-2}+\text { terms involving } \tau,
$$

viz. from this expression for $\Omega$ it appears that the curve $\Omega=0$ meets the line through 1,2 in the $n-2$ residues of these points, and moreover, for $(x, y, z)=\left(x_{1}, y_{1}, z_{1}\right)$ and therefore $(\rho, \sigma, \tau)=(\Delta, 0,0)$, the value of $\Omega$ is $=r .1^{n-1} 2$.

43. We can without difficulty write down an equation determining $\Omega^{\prime}$ as a function $(x, y, z)^{n-2}$, which, on putting therein $\tau=0$, becomes equal to the foregoing expression $\frac{1}{\Delta^{n-2}}\left(n .1^{n-1} 2, \ldots+\gamma \rho, \sigma\right)^{n-2}$, and which is moreover such that the curve $\Omega^{\prime}=0$ passes through the dps; which being so, we have as before, $\Omega=\Omega^{\prime}+(x, y, z)^{n-3} .012$, for the general value of $\Omega$.

To fix the ideas, consider the particular case $n=4$, the fixed curve a quartic: $\Omega^{\prime}$, on putting therein $\tau=0$, should become

$$
=\frac{1}{\Delta^{2}}\left(4 \cdot 1^{32}, 6 \cdot 1^{2} 2^{2}, 4 \cdot 12^{3}+\gamma \rho, \sigma\right)^{2} ;
$$

and it is to be shown that this will be the case if we determine $\Omega^{\prime}$, a quadric function of $(x, y, z)$, by the equation

$$
\left|\begin{array}{ccc}
(x, y, z)^{2} & \Omega^{\prime} \\
1\left(x_{1}, y_{1}, z_{1}\right)^{2} & , & 4.1^{32} \\
2\left(x_{1}, y_{1}, z_{1} \gamma x_{2}, y_{2}, z_{2}\right), & 6.1^{22^{2}} \\
1\left(x_{2}, y_{2}, z_{2}\right)^{2} & , & 4.12^{3} \\
a, b, c, f, g, h, & 0 \\
\vdots &
\end{array}\right|=0
$$

where the left-hand side is a determinant of seven lines and columns, the top line being $x^{2}, y^{2}, z^{2}, 2 y z, 2 z x, 2 x y, \Omega^{\prime}$, and similarly for the second line; the third line is 
$2 x_{1} x_{2}, 2 y_{1} y_{2}, 2 z_{1} z_{2}, 2\left(y_{1} z_{2}+y_{2} z_{1}\right), 2\left(z_{2} x_{1}+z_{1} x_{2}\right), 2\left(x_{1} y_{2}+x_{2} y_{1}\right), 6.1^{2} 2^{2}$, and in each of the last three lines we have six arbitrary constants followed by a 0 . The equation is of the form $\square+M \Omega^{\prime}=0$, where $\square$ is a quadric function $(x, y, z)^{2}$, and $M$ is a constant factor.

44. If the quartic curve has a dp, suppose at the point $\alpha$, coordinates $\left(x_{\alpha}, y_{\alpha}, z_{\alpha}\right)$, then in order that the curve $\Omega^{\prime}=0$ may pass through the $\mathrm{dp}$, we must for one of the last three lines substitute $\left(x_{\alpha}, y_{\alpha}, z_{a}\right)^{2}, 0$; and so for any other dp or dps of the quartic curve. And the conditions as to the dp or dps (if any) being satisfied in this manner, we may if we please, taking $\left(x_{\beta}, y_{\beta}, z_{\beta}\right)$ as the coordinates of an arbitrary point $\beta$ (not of necessity on the fixed curve), write any line not already so expressed, of the last three lines, in the form $\left(x_{\beta}, y_{\beta}, z_{\beta}\right)^{2}, 0$; the effect being to make the curve $\Omega^{\prime}=0$ pass through the arbitrary point $\beta$.

45. To show that the equation on putting therein $\tau=0$ does in fact give the required value, $\Omega^{\prime}=\frac{1}{\Delta^{2}}\left(4.1^{32}, 6.1^{2} 2^{2}, 4.12^{3}+\gamma \rho, \sigma\right)^{2},=\Phi$ suppose, it is to be observed that, effecting a linear substitution upon the first six columns, the equation may be written

$$
\begin{array}{ccc|}
(\rho, \sigma, \tau)^{2} & , & \Omega^{\prime} \\
1\left(\rho_{1}, \sigma_{1}, \tau_{1}\right)^{2} & , & 4.1^{32} \\
2\left(\rho_{1}, \sigma_{1}, \tau_{1} \gamma \rho_{2}, \sigma_{2},\right. & \left.\tau_{2}\right), & 6.1^{22^{2}} \\
1\left(\rho_{2}, \sigma_{2}, \tau_{2}\right)^{2} & , & 4.12^{3} \\
a^{\prime}, b^{\prime}, c^{\prime}, f^{\prime}, g^{\prime}, h^{\prime}, & 0
\end{array}
$$

where $\left(\rho_{1}, \sigma_{1}, \tau_{1}\right),\left(\rho_{2}, \sigma_{2}, \tau_{2}\right)$ are what $(\rho, \sigma, \tau)$ become on writing therein for $(x, y, z)$ the values $\left(x_{1}, y_{1}, z_{1}\right)$ and $\left(x_{2}, y_{2}, z_{2}\right)$ respectively; viz. we have $\left(\rho_{1}, \sigma_{1}, \tau_{1}\right)=(\Delta, 0,0)$; $\left(\rho_{2}, \sigma_{2}, \tau_{2}\right)=(0, \Delta, 0)$; the equation thus is

$$
\left|\begin{array}{ccccccc}
\rho^{2}, & \sigma^{2}, & \tau^{2}, & 2 \sigma \tau, & 2 \tau \rho, & 2 \rho \sigma, & \Omega^{\prime} \\
\Delta^{2}, & 0, & 0, & 0, & 0, & 0, & 4.1^{32} \\
0, & 0, & 0, & 0, & 0, & 2 \Delta^{2}, & 6.1^{22} 2^{2} \\
0, & \Delta^{2}, & 0, & 0, & 0, & 0, & 4.12^{3} \\
a^{\prime}, & b^{\prime}, & c^{\prime}, & f^{\prime}, & g^{\prime}, & h^{\prime}, & 0 \\
\vdots & & & & & &
\end{array}\right|=0
$$

and then by another linear substitution upon the columns, the last column can be changed into $\Omega^{\prime}-\Phi, 0,0,0,0,0,0$; whence writing $\tau=0$, the equation becomes

$$
\left|\begin{array}{rrrrrrr}
\rho^{2}, & \sigma^{2}, & 0, & 0, & 0, & 2 \rho \sigma, & \Omega^{\prime}-\Phi \\
\Delta^{2}, & 0, & 0, & 0, & 0, & 0, & 0 \\
0, & 0, & 0, & 0, & 0, & 2 \Delta^{2}, & 0 \\
0, & \Delta^{2}, & 0, & 0, & 0, & 0, & 0 \\
a^{\prime}, & b^{\prime}, & c^{\prime}, & f^{\prime}, & g^{\prime}, & h^{\prime}, & 0 \\
: & & & & & &
\end{array}\right|=0,
$$


or, omitting a constant factor, it is

$$
\left|\begin{array}{cccc}
\rho^{2}, & \sigma^{2}, & 2 \rho \sigma, & \Omega^{\prime}-\Phi \\
\Delta^{2}, & 0, & 0, & 0 \\
0, & 0, & 2 \Delta^{2}, & 0 \\
0, & \Delta^{2}, & 0, & 0
\end{array}\right|=0,
$$

that is, $\Omega^{\prime}-\Phi=0$, or $\Omega^{\prime}=\Phi,=\frac{1}{\Delta^{2}}\left(4.1^{32}, 6.1^{2} 2^{2}, 4.12^{3}+\gamma \rho, \sigma\right)^{2}$, the required value.

46. Considering the equation for $\Omega^{\prime}$ as expressed in the before-mentioned form $\square+M \Omega^{\prime}=0$, the value of the constant factor $M$ is

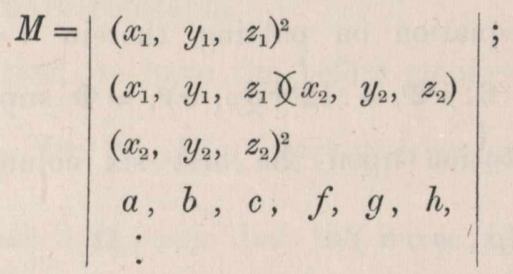

or if, instead of each line such as $a, b, c, f, g, h$, we have a line $\left(x_{a}, y_{a}, z_{a}\right)^{2}$, then we have

a value which is

$$
M=\left|\begin{array}{l}
\left(x_{1}, y_{1}, z_{1}\right)^{2} \\
\left(x_{1}, y_{1}, z_{1} \chi x_{2}, y_{2}, z_{2}\right) \\
\left(x_{2}, y_{2}, z_{2}\right)^{2} \\
\left(x_{\alpha}, y_{\alpha}, z_{\alpha}\right)^{2} \\
\left(x_{\beta}, y_{\beta}^{\prime}, z_{\beta}\right)^{2} \\
\left(x_{\gamma}, y_{\gamma}, z_{\gamma}\right)^{2}
\end{array}\right|,
$$

$$
=\left|\begin{array}{lll}
x_{1}, & y_{1}, & z_{1} \\
x_{2}, & y_{2}, & z_{2} \\
x_{\alpha}, & y_{\alpha}, & z_{\alpha}
\end{array}\right|\left|\begin{array}{lll}
x_{1}, & y_{1}, & z_{1} \\
x_{2}, & y_{2}, & z_{2} \\
x_{\beta}, & y_{\beta}, & z_{\beta}
\end{array}\right|\left|\begin{array}{ccc}
x_{1}, & y_{1}, & z_{1} \\
x_{2}, & y_{2}, & z_{2} \\
x_{\gamma}, & u_{\gamma}, & z_{\gamma}
\end{array}\right|\left|\begin{array}{lll}
x_{\alpha}, & y_{a}, & z_{\alpha} \\
x_{\beta}, & y_{\beta}, & z_{\beta} \\
x_{\gamma}, & y_{\gamma}, & z_{\gamma}
\end{array}\right|,
$$

or say this is $=12 \alpha .12 \beta .12 \gamma \cdot \alpha \beta \gamma$.

47. It is obvious that the foregoing process is applicable to the general case of the fixed curve of the order $n$ with $\delta$ dps, and gives always $\Omega^{\prime}$, by an equation of the foregoing form $\square+M \Omega^{\prime}=0$, where $\square$ is a function $(x, y, z)^{n-2}$ of the coordinates, and $M$ is a constant factor. Supposing that in the determinant for $\Omega^{\prime}$, each of the lower lines is written in the form $\left(x_{\alpha}, y_{\alpha}, z_{\alpha}\right)^{n-2}, 0$, the number of the points $\alpha$ is $=\frac{1}{2}(n-1)(n-2)$, viz. these are the $\delta \mathrm{dps}$, and $\frac{1}{2}(n-1)(n-2)-\delta,=p$, other points $\alpha$. The general expression of $M$ is $M=12 \alpha .12 \beta \ldots\left(\alpha^{n-3} \beta^{n-3} \ldots\right)$, viz. equating to zero a factor such as $12 \alpha$, this expresses that the point $\alpha$ is on the line 12 ; but equating to zero the last factor $\left(\alpha^{n-3} \beta^{n-3} \ldots\right)$, this expresses that the several points $\alpha$, viz. the dps and the $p$ other points $\alpha$, are on a curve of the order $n-3$. 
48. Preceding the case $n=4$, above considered, we have, of course, the case $n=3$, $\delta=0$, the fixed curve a cubic; the equation for $\Omega^{\prime}$ is here

giving

$$
\left|\begin{array}{cccc}
x, & y, & z, & \Omega^{\prime} \\
x_{1}, & y_{1}, & z_{1}, & 3.1^{22} \\
x_{2}, & y_{2}, & z_{2}, & 3.12^{2} \\
x_{a}, & y_{a}, & z_{a} &
\end{array}\right|=0
$$

$$
\frac{1}{3} \Omega^{\prime}=\frac{1^{22} \cdot 02 \alpha+12^{2} \cdot 0 \alpha 1}{12 \alpha}
$$

or if we write herein 3 for $\alpha$, this is

$$
\frac{1}{3} \Omega^{\prime}=\frac{122.023+12^{2} \cdot 031}{123}
$$

and we have hence the general form

$$
\frac{1}{3} \Omega=\frac{1^{22} \cdot 023+12^{2} \cdot 031}{123}+K .012
$$

where $K$ is an arbitrary constant.

49. There is, however, a more simple particular solution $\frac{1}{3} \Omega^{\prime}=$ polar function $012\left(f=x^{3}+y^{3}+z^{3}\right.$, then $\left.012=x x_{1} x_{2}+y y_{1} y_{2}+z z_{1} z_{2}\right)$, which, to avoid a confusion of notation, we may write $=\widetilde{012}$. We at once verify this; for, expressing the coordinates $(x, y, z)$ in terms of $(\rho, \sigma, \tau)$, we have $\frac{1}{3} \Omega^{\prime}=\widetilde{012},=\frac{1}{\Delta}\left(1^{22} \cdot \rho+12^{2} \cdot \sigma+\widetilde{123} \cdot \tau\right)$, which, for $\tau=0$ becomes $=\frac{1}{\Delta}\left\{1^{22} \cdot \rho+12^{2} \cdot \sigma\right\}$.

We must, of course, have an identity of the form

$$
\widetilde{012}=\frac{1^{22} \cdot 023+12^{2} \cdot 031}{123}+K .012 \text {, }
$$

and to find $K$, writing here $0=3$, we have $K=\frac{\widetilde{123}}{123}$, or we have the identity

$$
123 \widetilde{012}-\widetilde{123} 012=1^{22} .023+12^{2} .031
$$

Single Letter Notation for the Polar Functions of the Cubic. Art. Nos. 50 and 51.

50. The notation of single letters for the polar functions is not much required in the case of the cubic, but, in the next following case of the quartic it can hardly be dispensed with, and I therefore establish it in the case of the cubic: viz. I write

$$
2^{2} 3,3^{2} 1,1^{2} 2=f, g, h, 23^{2}, 31^{2}, 12^{2}=i, j, k ; \widetilde{123}=l,
$$

or, what is the same thing, the expression for the cubic function $f$ in terms of $\rho, \sigma, \tau$ is

$$
\Delta^{3} \cdot f=3 h \rho^{2} \sigma+3 j \rho^{2} \tau+3 k \rho \sigma^{2}+6 l \rho \sigma \tau+3 g \rho \tau^{2}+3 f \sigma^{2} \tau+3 i \sigma \tau^{2} ;
$$


an equation which, writing $0^{3}$ instead of $f$, may also be written

$$
\Delta^{3} \cdot 0^{3}=\left(3 h, 3 j, 3 k, 6 l, 3 g, 3 f, 3 i \chi \rho^{2} \sigma, \rho^{2} \tau, \rho \sigma^{2}, \rho \sigma \tau, \rho \tau^{2}, \sigma^{2} \tau, \sigma \tau^{2}\right) ;
$$

and $I$ join to it the series of equations

$$
\begin{aligned}
& \Delta^{2} \cdot 0^{2} 1=\left(0,2 h, 2 j, k, 2 l, g \chi \rho^{2}, \rho \sigma, \rho \tau, \sigma^{2}, \sigma \tau, \tau^{2}\right), \\
& \text { " } 0^{2} 2=(h, 2 k, 2 l, 0,2 f, i \gamma \quad \text { ", } \\
& \text { " } 0^{2} 3=(j, 2 l, 2 g, f, 2 i, 0 \gamma \quad \text { ", }) \text {, } \\
& \Delta .01^{2}=(0, h, j \gamma \rho, \sigma, \tau), \\
& \Delta \widetilde{012}=(h, k, l \gamma \quad, \quad) \text {, } \\
& \text { " } \widetilde{013}=(j, l, g \gamma \quad, \quad) \text {, } \\
& \text { " } 02^{2}=(k, 0, f \gamma \quad, \quad) \text {, } \\
& \text { " } \widetilde{023}=(l, f, i \gamma \quad, \quad) \text {, } \\
& \text { " } 03^{2}=(g, i, 0 \gamma \quad \text {, }) \text {. }
\end{aligned}
$$

51. In particular, we have $\Delta . \widetilde{012}=h \rho+k \sigma+l \tau$, and the above-mentioned identity $123 \widetilde{012}-\widetilde{123} 012=122.023+12^{2} .031$ is simply $h \rho+k \sigma+l \tau-l \tau=h \rho+k \sigma$.

Single Letter Notation for the Polar Functions of the Quartic. Art. No. 52.

52. I write here

$$
2^{3} 3,3^{3} 1,1^{32}=f, g, h ; 23^{3}, 31^{3}, 12^{3}=i, j, k ;
$$

$1223,12^{23}, 123^{2}=l, m, n ; 2^{2} 3^{2}, 3^{2} 1^{2}, 1^{2} 2^{2}=p, q, r ;$

so that the expression for the quartic function $f$ in terms of $\rho, \sigma, \tau$ is

$$
\begin{aligned}
\Delta^{4} \cdot f=4 h \rho^{3} \sigma+4 j \rho^{3} \tau+ & 6 p \rho^{2} \sigma^{2}+12 l \rho^{2} \sigma \tau+6 q \rho^{2} \tau^{2} \\
& +4 k \rho \sigma^{3}+12 m \rho \sigma^{2} \tau+12 n \rho \sigma \tau^{2}+4 g \rho \tau^{3}+4 f \sigma^{3} \tau+6 r \sigma^{2} \tau^{2}+4 i \sigma \tau^{3}
\end{aligned}
$$

which, putting $0^{4}$ for $f$, may also be written

$$
\begin{aligned}
\Delta^{4} .0^{4}=(4 h, 4 j ; 6 p, 12 l, 6 q: 4 k, 12 m, 12 n, 4 g: 4 f, 6 r, 4 i \dagger) \\
\\
\left(\rho^{3} \sigma, \rho^{3} \tau ; \rho^{2} \sigma^{2}, \rho^{2} \sigma \tau, \rho^{2} \tau^{2}, \rho \sigma^{3}, \rho \sigma^{2} \tau, \rho \sigma \tau^{2}, \rho \tau^{3}, \sigma^{3} \tau, \sigma^{2} \tau^{2}, \sigma \tau^{3}\right) ;
\end{aligned}
$$

and $I$ join to it the series of equations

$\Delta^{3} .0^{3} 1=\left(0 ; 3 h, 3 j ; 3 r, 6 l, 3 q ; k, 3 m, 3 n, g \gamma \rho^{3}, \rho^{2} \sigma, \rho^{2} \tau, \rho \sigma^{2}, \rho \sigma \tau, \rho \tau^{2}, \sigma^{3}, \sigma^{2} \tau, \sigma \tau^{2}, \tau^{3}\right)$,

„ $0^{32} 2=(h ; 3 r, 3 l ; 3 k, 6 m, 3 n ; 0,3 f, 3 p, i \gamma$

” $0^{3} 3=(j ; 3 l, 3 q ; 3 n, 6 n, 3 g ; f, 3 p, 3 i, 0 \gamma$ 


$$
\begin{aligned}
& \Delta^{2} \cdot 0^{2} 1^{2}=\left(0 ; 2 h, 2 j ; r, 2 l, q \gamma \rho^{2} ; \rho \sigma, \rho \tau ; \sigma^{2}, \sigma \tau, \tau^{2}\right) \text {, } \\
& \text { " } 0^{2} 12=(h ; 2 r, 2 l ; k, 2 m, n \gamma \quad \text { ", ), } \\
& \text { " } 0^{2} 13=(j ; 2 l, 2 q ; m, 2 n, g \gamma \quad \text { ", }) \text {, } \\
& \text { " } 0^{2} 2^{2}=(r ; 2 k, 2 m ; 0,2 f, p \gamma \quad \text { " ), } \\
& \text { " } 0^{2} 23=(l ; 2 m, 2 n ; f, 2 p, i \gamma \quad \text { " }) \text {, } \\
& \text { " } 0^{2} 3^{2}=(q ; 2 n, 2 g ; p, 2 i, 0 \gamma \quad ",) \text {, } \\
& \Delta \cdot 01^{3}=(0, h, j \gamma \rho, \sigma, \tau), \\
& \text { "0122 }=(h, r, l \gamma \quad \text { »), } \\
& \text { " } 01^{2} 3=(j, l, q \gamma \quad, \quad) \text {, } \\
& \text { "012 }=(r, k, m \chi \quad, \quad) \text {, } \\
& \text { " } 0123=(l, m, n \gamma \quad \text { " }) \text {, } \\
& \text { " } 013^{2}=(q, n, g \gamma \quad, \quad) \text {, } \\
& \text { " } 02^{3}=(k, 0, f \gamma \quad, \quad) \text {, } \\
& \text { " } 02{ }^{2} 3=(m, f, p \gamma \quad \text { ” }) \text {, } \\
& \text { " } 023^{2}=(n, p, i \gamma \quad ") \text {, } \\
& \text { " } 03^{3}=(g, i, 0 \gamma \quad,) \text {, }
\end{aligned}
$$

which will be convenient in the sequel.

Major Function-The Fixed Curve a Cubic. Art. No. 53.

53. It has been already seen that a simple particular form is $\frac{1}{3} \Omega^{\prime}=\widetilde{012}$ : and that the general form is $\Omega=\Omega^{\prime}+K .012$.

\section{Major Function-The Fixed Curve a Quartic. Art. No. 54.}

54. It is to be shown that a particular form is

$$
\frac{1}{2} \Omega^{\prime}=\frac{-01^{3} \cdot 02^{3}+01^{22} \cdot 012^{2}+0^{2} 12 \cdot 1^{22} 2^{2}}{1^{2} 2^{2}} .
$$

In fact, by the foregoing values of $\Delta .01^{3}$, \&c., the numerator of this expression, multiplied by $\Delta^{2}$, is $=$

which is

$$
\begin{aligned}
& -(h \sigma+j \tau)(k \rho+f \tau) \\
& +(h \rho+r \sigma+l \tau)(r \rho+k \sigma+m \tau) \\
& +r\left(h \rho^{2}+2 r \rho \sigma+2 l \rho \tau+k \sigma^{2}+2 m \sigma \tau+n \tau^{2}\right)
\end{aligned}
$$

$$
=2 h r \rho^{2}+3 r^{2} \rho \sigma+(h m-j k+3 l r) \rho \tau+2 k r \sigma^{2}+(-f h+k l+3 m r) \sigma \tau+(-f j+l m+n r) \tau^{2} ;
$$

and this, for $\tau=0$, becomes

$$
=r\left(2 h \rho^{2}+3 r \rho \sigma+2 k \sigma^{2}\right) .
$$

C. XII. 
Hence for $\tau=0$, we have

$$
\Omega^{\prime}=\frac{1}{\Delta^{2}}\left(4 h \rho^{2}+6 r \rho \sigma+4 k \sigma^{2}\right)
$$

that is,

$$
=\frac{1}{\Delta^{2}}\left\{4 \cdot 1^{32} \cdot \rho^{2}+6 \cdot 1^{2} 2^{2} \cdot \rho \sigma+4.12^{3} \cdot \sigma^{2}\right\}
$$

and $\Omega^{\prime}$ is thus a form of the major function $(x, y, z)_{12}{ }^{2}$. Of course the general form is $\Omega=\Omega^{\prime}+(x, y, z)^{1} .012$.

Syzygy of the Major Function. Art. No. 55.

55. Writing now $(x, y, z)_{12}{ }^{n-2}=\Omega_{12}$; and taking on the fixed curve a new point 3 , consider the like functions $\Omega_{23}$ and $\Omega_{31}$ : it is to be shown that we have identically

$$
\Omega_{23} .031 .012+\Omega_{31} .012 .023+\Omega_{12} .023 .031-(123)^{2} f=023.031 .012(x, y, z)^{n-3},
$$

where $(x, y, z)^{n-3}$ is a properly determined minor function; or, considering herein 0 as a point on the fixed curve and writing therefore $f=0$, the equation is

$$
\frac{\Omega_{23}}{023}+\frac{\Omega_{31}}{031}+\frac{\Omega_{12}}{012}=(x, y, z)^{n-3 . *}
$$

56. Write for a moment $X=\Omega_{23} .031 .012+\Omega_{31} .012 .023+\Omega_{12} .023 .031$; then $k$ being an arbitrary coefficient, we have $X-k f=0$, a curve of the order $n$, passing through the points $1,2,3$, and also through the residues of 2,3 , the residues of 3,1 , and the residues of 1,2 ; in fact, at the point 1 we have $012=0,031=0$, and therefore $X=0$; also $f=0$; and therefore 1 is a point on the curve. Again, at any residue of 2,3 , we have $\Omega_{23}=0,023=0$, and therefore $X=0$; also $f=0$; and hence the residue of 2,3 is a point on the curve.

It is next to be shown that $k$ can be so determined that the curve $X-k f=0$ shall have a $d p$ at each of the points 1, 2, 3. Supposing this to be so, we have the line 23 meeting the curve $X-k f=0$ in the points 2 and 3 , each counting twice, and in the $n-2$ residues of 2,3 , that is, in $n+2$ points; hence the curve $X-k f=0$ must contain as part of itself the line 23 , and similarly it must contain as part of itself each of the other lines 31 and 12, viz. we shall then have $X-k f=023.031 .012 .(x, y, z)^{n-3}$; and from this equation observing that the curves $\Omega_{23}=0, \Omega_{31}=0, \Omega_{12}=0$ each pass through the dps, it follows that the curve $(x, y, z)^{n-3}=0$ also passes through the dps; hence, $k$ being found to be $=(123)^{2}$, the theorem will be proved.

57. Taking an arbitrary point $\alpha$ coordinates $\left(x_{a}, y_{a}, z_{a}\right)$, and writing

$$
D=x_{a} \frac{d}{d x}+y_{a} \frac{d}{d y}+z_{a} \frac{d}{d z}
$$

* This is the differential theorem corresponding to C. and G.'s integral theorem, p. 26, viz. this is $S_{\xi \eta}+S_{\eta \zeta}+S_{\zeta \xi}=I$, a sum of three integrals of the third kind=an integral of the first kind. 
we have to find $k$, so that the curve $D(X-k f)=0$ shall pass through the point 1 . Observing that $D 023=\alpha 23$, \&c., we have

$$
\begin{aligned}
D(X-k f)= & D \Omega_{23} .031 .012+\Omega_{23}(031 . \alpha 12+\alpha 31.012) \\
& +\alpha 23\left(\Omega_{31} \cdot 012+\Omega_{12} \cdot 031\right) \\
& +023\left\{\Omega_{31} \cdot \alpha 12+\Omega_{12} . \alpha 31+D \Omega_{31} \cdot 012+D \Omega_{12} .031\right\} \\
& -k D f,
\end{aligned}
$$

and, to make the curve pass through 1 , writing herein $0=1$, we have

$$
0=123\left(\Omega_{\mathrm{s}^{1}}{ }^{1} \cdot \alpha 12+\Omega_{12}{ }^{1} \cdot \alpha 31\right)-k(D f)^{1},
$$

where the superfix (1) denotes that we are in $\Omega_{\mathrm{s1}}, \Omega_{12}$ and $D f$ respectively to write $0=1$. We have $\Omega_{31}{ }^{1}=n .1^{n-1} 3, \Omega_{12}{ }^{1}=n .1^{n-1} 2,(D f)^{1}=n .1^{n-1} \alpha$, and the equation thus is

$$
n .123\left(1^{n-1} 3 . \alpha 12+1^{n-1} 2 . \alpha 31\right)-k n .1^{n-1} \alpha=0 .
$$

But we have identically $1^{n-1} 1 . \alpha 23+1^{n-1} 2 . \alpha 31+1^{n-1} 3 . \alpha 12=1^{n-1} \alpha \cdot 123$, where $1^{n-1} 1,=1^{n}$ is; in fact, $=0$; the factor $1^{n-1} \alpha$ thus divides out, and the equation becomes $k=(123)^{2}$; viz. $k$ having this value, the curve $X-k f=0$ will have a dp at 1 ; and clearly by symmetry, it will also have a dp at 2 , and at 3 ; the theorem is thus proved.

\section{The Syzygy, Fixed Curve a Cubic. Art. No. 58.}

58. The syzygy may be verified independently in the case where the fixed curve is a cubic. Observe that the syzygy, if satisfied for any particular form of $\Omega$, will be generally satisfied; we may therefore take $\frac{1}{3} \Omega_{12}=\widetilde{012}$. Writing then

$$
\frac{\frac{1}{3} \Omega_{12}}{012}=\frac{\widetilde{012}}{012},=\{012\} \text { suppose, }
$$

and taking 0 to be a point on the cubic curve, we ought to have $\{023\}+\{031\}+\{012\}=\mathrm{a}$ constant; the value of this constant comes out to be $=\{123\}$, and the syzygy in its complete form thus is

We have

$$
\{023\}+\{031\}+\{012\}=\{123\} .
$$

$$
\Delta \widetilde{023}, \Delta \widetilde{031}, \Delta \widetilde{012}=l \rho+f \sigma+i \tau, j \rho+l \sigma+g \tau, h \rho+k \sigma+l \tau,
$$

and the equation thus is

$$
\frac{l \rho+f \sigma+i \tau}{\rho}+\frac{j \rho+l \sigma+g \tau}{\sigma}+\frac{h \rho+k \sigma+l \tau}{\tau}-l=0 ;
$$

this, multiplied by $\rho \sigma \tau$, becomes

$$
h \rho^{2} \sigma+j \rho^{2} \tau+k \rho \sigma^{2}+2 l \rho \sigma \tau+g \rho \tau^{2}+f \sigma^{2} \tau+i \sigma \tau^{2}=0,
$$

which is, in fact, $\frac{1}{3} f=0$, the equation of the cubic curve.

Observe that the new symbol $\{012\}$ is, in virtue of its determinant denominator, an alternate function, $\{012\}=-\{102\},\{012\}=\{120\}=\{201\}$. The syzygy is a relation between any four points $1,2,3,0$ of the curve, and it may be also expressed in the form

$$
\{123\}-\{230\}+\{301\}-\{012\}=0 .
$$


The Syzygy, Fixed Curve a Quartic. Art. No. 59.

59. Taking $\Omega_{12}$ as before, we have

$$
\frac{\frac{1}{2} \Omega_{12}}{012}=\frac{-01^{3} \cdot 02^{3}+01^{22} \cdot 012^{2}+0^{2} 12 \cdot 1^{2} 2^{2}}{012 \cdot 1^{2} 2^{2}}=\left\{0^{2} 12\right\} \text { suppose : }
$$

and then, taking 0 to be a point on the quartic curve, we ought to have

$$
\left\{0^{2} 23\right\}+\left\{0^{2} 31\right\}+\left\{0^{2} 12\right\}=(x, y, z)^{1} \text {, a linear function of }(x, y, z),
$$

or, what is the same thing, considering the left-hand side as expressed in terms of $\rho, \sigma, \tau$, the sum should be

$$
=(\rho, \sigma, \tau)^{1} \text {, a linear function of }(\rho, \sigma, \tau) .
$$

By a preceding formula we have

$$
\begin{aligned}
\left\{0^{2} 12\right\}=\frac{1}{\Delta^{2} r \tau}\left\{2 h r \rho^{2}+3 r^{2} \rho \sigma+\right. & h m-j k+3 l r) \rho \tau \\
& \left.+2 k r \sigma^{2}+(-f h+k l+3 m r) \sigma \tau+(-f j+l m+n r) \tau^{2}\right\},
\end{aligned}
$$

which is

$$
=\frac{1}{\Delta^{2}}\left\{\left(3 l+\frac{h m-j k}{r}\right) \rho+\left(3 m+\frac{-f h+k l}{r}\right) \sigma+\left(n+\frac{-f j+l m}{r}\right) \tau\right\}+\frac{1}{\Delta^{2}} \frac{2 h \rho^{2}+3 r \rho \sigma+2 k \sigma^{2}}{\tau} .
$$

And hence, forming the sum $\left\{0^{2} 23\right\}+\left\{0^{2} 31\right\}+\left\{0^{2} 12\right\}$, we have first a fractional part which is found to be integral, viz. this is

$$
\begin{aligned}
\frac{1}{\Delta^{2}} & \left\{\frac{2 f \sigma^{2}+3 p \sigma \tau+2 i \tau^{2}}{\rho}+\frac{2 g \tau^{2}+2 q \tau \rho+2 j \rho^{2}}{\sigma}+\frac{2 h \rho^{2}+3 r \rho \sigma+2 k \sigma^{2}}{\tau}\right\}, \\
& =\frac{1}{\Delta^{2} \rho \sigma \tau}\left\{2 h \rho^{3} \sigma+2 j \rho^{3} \tau+3 r \rho^{2} \sigma^{2}+3 q \rho^{2} \tau^{2}+2 k \rho \sigma^{3}+2 g \rho \tau^{3}+2 f \sigma^{3} \tau+3 p \sigma^{2} \tau^{2}+2 i \sigma \tau^{3}\right\}, \\
& =\frac{1}{\Delta^{2} \rho \sigma \tau}\left\{\frac{1}{2} \Delta^{4} f-6 l \rho^{2} \sigma \tau-6 m \rho \sigma^{2} \tau-6 n \rho \sigma \tau^{2}\right\}
\end{aligned}
$$

or since $f=0$, this is

$$
=\frac{1}{\Delta^{2}}(-6 l \rho-6 m \sigma-6 n \tau) .
$$

We then have integral terms which are at once deduced from the above integral terms of $0^{2} 12$; collecting the several terms, we find

$$
\begin{aligned}
&\left\{0^{2} 23\right\}+\left\{0^{2} 31\right\}+\left\{0^{2} 12\right\}= \\
& \frac{1}{\Delta^{2}}\left\{\rho\left(l+\frac{m n-g k}{p}+\frac{j n-g h}{q}+\frac{h m-j k}{r}\right)+\sigma\left(m+\frac{f n-i k}{p}+\frac{l n-h i}{q}+\frac{l l-f h}{r}\right)\right. \\
&\left.+\tau\left(n+\frac{l m-f g}{p}+\frac{g l-i j}{q}+\frac{l m-f j}{r}\right)\right\}
\end{aligned}
$$

which is the required result. 
Preparation for the Conversion-The Symbol J. Art. Nos. 60 to 63.

60. I use $\partial$ as the symbol of a quasi-differentiation, viz. $U$ being any function of $(x, y, z), \partial U$ denotes $\frac{1}{d \omega}$ multiplied by the differential $\frac{d U}{d x} d x+\frac{d U}{d y} d y+\frac{d U}{d z} d z$; in such a differential, the increments $d x, d y, d z$ do not in general present themselves in the combinations $y d z-z d y, z d x-x d z, x d y-y d x$; but they will do so if $U$ is a function of the degree zero in the coordinates $x, y, z$ (that is, if $U$ be the quotient of two homogeneous functions of the same degree); and this being so, we can by the equations

$$
\frac{y d z-z d y}{\frac{d f}{d x}}=\frac{z d x-x d z}{\frac{d f}{d y}}=\frac{x d y-y d x}{\frac{d f}{d z}},=d \omega
$$

get rid of the increments, and $\partial U$ will denote a function of $(x, y, z)$ derived in a definite manner from the function $U$. The symbol $\partial$ will be used only in the case in question of a function of the degree zero. Of course $\partial_{1}$ will denote the like operation in regard to $\left(x_{1}, y_{1}, z_{1}\right)$; and so $\partial_{2}$, \&c.; and we may for greater clearness write $\partial_{0}$ in place of $\partial$.

61. Consider then $\partial \frac{P}{Q}$, where $P, Q$ are functions $(x, y, z)^{m}$ of the same degree: we have

and then

$$
\partial \frac{P}{Q}=\frac{1}{Q^{2} d \omega}(Q d P-P d Q)
$$

$$
d P=\frac{d P}{d x} d x+\frac{d P}{d y} d y+\frac{d P}{d z} d z, \quad \frac{1}{m} P=\frac{d P}{d x} x+\frac{d P}{d y} y+\frac{d P}{d z} z,
$$

with the like formulæ for $Q$. Substituting, we find

$$
\partial \frac{P}{Q}=\frac{1}{m Q^{2} d \omega}\left\{\frac{d(Q, P)}{d(y, z)}(y d z-z d y)+\frac{d(Q, P)}{d(z, x)}(z d x-x d z)+\frac{d(Q, P)}{d(x, y)}(x d y-y d x)\right\},
$$

that is,

or say

$$
\partial \frac{P}{Q}=\frac{1}{m Q^{2}}\left\{\frac{d f}{d x} \frac{d(Q, P)}{d(y, z)}+\& c .\right\}=\frac{1}{m Q^{2}} \frac{d(f, Q, P)}{d(x, y, z)},=\frac{1}{m Q^{2}} J(f, Q, P)
$$

62. As an example, consider

$$
\partial \frac{P}{Q}=-\frac{1}{m Q^{2}} J(P, Q, f) .
$$

The determinant is

$$
\left.\partial\{012\},=-\frac{1}{(012)^{2}} J \widetilde{012}, 012, f\right)
$$

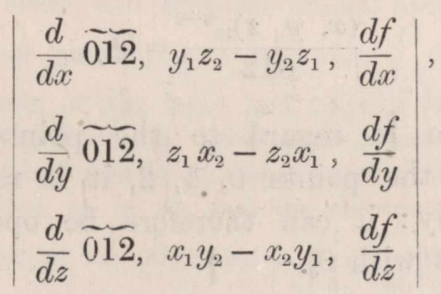


and the coefficient herein of $\frac{d}{d x} \widetilde{012}$ is $\left(z_{1} x_{2}-z_{2} x_{1}\right) \frac{d f}{d z}-\left(x_{1} y_{2}-x_{2} y_{1}\right) \frac{d f}{d y}$, which is

$$
=x_{2}\left(x_{1} \frac{d f}{d x}+y_{1} \frac{d f}{d y}+z_{1} \frac{d f}{d z}\right)-x_{1}\left(x_{2} \frac{d f}{d x}+y_{2} \frac{d f}{d y}+z_{2} \frac{d f}{d z}\right),=3\left(0^{2} 1 . x_{2}-0^{2} 2 . x_{1}\right)
$$

and so for the other terms.

The determinant is thus

$$
=3\left[0^{2} 1\left(x_{2} \frac{d}{d x}+y_{2} \frac{d}{d y}+z_{2} \frac{d}{d z}\right)-0^{22}\left(x_{1} \frac{d}{d x}+y_{1} \frac{d}{d y}+z_{1} \frac{d}{d z}\right)\right] \widetilde{012}
$$

say this is

$$
=3\left[0^{2} 1 .\left[^{2}\right)-0^{2} 2 \text { ID }\right] \widetilde{012} \text {. }
$$

But we have $13012=12^{2}, \widetilde{012} \widetilde{012}=1^{22}$, and the determinant is then $=3\left(0^{2} 1.12^{2}-0^{2} 2.1^{22}\right)$; whence finally, writing $\partial_{0}$ instead of $\partial$,

$$
\partial_{0}\{012\}=-3 \cdot \frac{0^{2} 1 \cdot 12^{2}-0^{2} 2 \cdot 1^{22}}{(012)^{2}} .
$$

63. By cyclical interchange of the $0,1,2$, we have

$$
\begin{aligned}
& \partial_{1}\{012\}=-3 \cdot \frac{1^{22} \cdot 022-01^{2} \cdot 02^{2}}{(012)^{2}} \\
& \partial_{2}\{012\}=-3 . \frac{02^{2} \cdot 01^{2}-12^{2} \cdot 0^{2} 1}{(012)^{2}}
\end{aligned}
$$

and thence adding, we find

$$
\left(\partial_{0}+\partial_{1}+\partial_{2}\right)\{012\}=0,
$$

an important property which, joined to the equation before obtained,

$$
\{023\}+\{031\}+\{012\}=\{123\},
$$

completes the theory of the function $\{012\}$.

Conversion of the Major Function (Interchange of Limits and Parametric Points).

Art. No. 64 .

64. Write in general

$$
\frac{(x, y, z)_{12}{ }^{n-2}}{012}=Q_{0,12}
$$

$Q_{0,12}$ is an alternate function in regard to the points $1,2\left(Q_{0,12}=-Q_{0,21}\right)$; and in regard to the coordinates of the points $0, .1,2$, it is rational, but not integral, of the degrees $n-3,0,0$ respectively: it can therefore be operated upon with $\partial_{1}$ or $\partial_{2}$, but (except in the case $n=3$ ) not with $\partial_{0}$. 
The conversion relates not to the general major function $(x, y, z)_{12}{ }^{n-2}$, but to this function with the arbitrary constants properly determined, and consists in a relation between two functions $Q_{4,12}$ and $Q_{1,45}$ (each of them a function of three out of four arbitrary points $1,2,4,5$ on the fixed curve), viz. the conversion is

$$
\partial_{1} Q_{4,12}=\partial_{4} Q_{1,45}
$$

an equation which may be written in four different forms, viz. we may in the form written down interchange 1,2 and also 4,5 .*

The determination of the constants is a very peculiar one, inasmuch as it is not algebraical; viz. in the case of the cubic curve, about to be considered, it appears that $Q_{0,12}$ contains the term $\int_{2}^{1} d \omega \partial_{3}\{036\}$, which is a transcendental function of the coordinates of the parametric points 1 and 2 .

The Conversion, Fixed Curve a Cubic. Art. No. 65.

65. We may write $Q_{0,12}=\{012\}+K$, where $K$ is a constant, that is, it is independent of the point 0 , but depends on the parametric points 1 and 2 . I assume $K$ to be properly determined, and give an $\grave{a}$ posteriori verification of the equation $\partial_{1} Q_{4,12}=\partial_{4} Q_{1,45}$. The value is $K=\int_{2}^{1} d \omega \partial_{3}\{036\}-\{123\}$, where 3,6 are arbitrary points on the cubic curve, and where in the definite integral, regarded as an integral $\int U d u$ with a current variable $u$, the meaning is that this variable has at the limits the values $u_{1}, u_{2}$ which belong to the points 1 and 2 respectively: a fuller explanation might be proper, but the investigation will presently be given in a form not depending on any integral at all.

Substituting for $K$ its value, we have

or, as this may also be written,

$$
Q_{0,12}=\{012\}+\left[\int_{2}^{1} d \omega \partial_{3}\{036\}-\{123\}\right]
$$

We have thence

$$
=-\{023\}-\{031\}+\int_{2}^{1} d \omega \partial_{3}\{036\} .
$$

$$
\partial_{1} Q_{0,12}=-\partial_{1}\{031\}+\partial_{3}\{136\}
$$

* The meaning of the property is better seen from the integral form: $Q_{0,12}$ is a function of the points $0,1,2$ and $Q_{0,45}$ the like function of the points $0,4,5$ such that $\int_{5}^{4} d \omega Q_{0,12}=\int_{2}^{1} d \omega Q_{0,45}$; which equation operated upon with $\partial_{1} \partial_{4}$ gives the formula of the text. And there is thus the meaning (alluded to in the heading) that there exists for the integral of the third kind a canonical form (C. and G.'s endliche Normalform), such that the integral is not altered by the interchange of the limits and the parametric points. The expression for $Q_{0,12}$ mentioned further on in the text for the case, fixed curve a cubic, shows that in this case the canonical form of the integral of the third kind is $\int_{5}^{4} d \omega\left[\{012\}+\left(\int_{2}^{1} d \omega \partial_{3}\{036\}-\{123\}\right)\right]$. 
and consequently

$$
\begin{aligned}
& \partial_{1} Q_{4,12}=-\partial_{1}\{431\}+\partial_{3}\{136\}, \\
& \partial_{4} Q_{1,45}=-\partial_{4}\{134\}+\partial_{3}\{436\} ;
\end{aligned}
$$

hence, observing that $\{431\}=-\{134\}$, \&c., we have

$$
\begin{aligned}
\partial_{1} Q_{4,12}-\partial_{4} Q_{1,45} & =\left(\partial_{1}+\partial_{4}\right)\{134\}+\partial_{3}\{136\}-\partial_{3}\{436\}, \\
& =-\partial_{3}\{134\}+\partial_{3}\{136\}-\partial_{3}\{436\},
\end{aligned}
$$

which, observing that we have $\partial_{3}\{641\}=0$, is

the required theorem.

$$
=\partial_{3}(\{136\}-\{364\}+\{641\}-\{413\}),=0,
$$

To avoid, in the proof, the use of the integral sign, we have only to consider the required function $Q_{0,12}$ as given by the foregoing differential formula

$$
\partial_{1} Q_{0,12}=-\partial_{1}\{031\}+\partial_{3}\{136\},
$$

for we have then the values of $\partial_{1} Q_{4,12}$ and $\partial_{4} Q_{1,45}$ : the rest of the proof the same as before.

The Conversion, Fixed Curve a Quartic. Art. Nos. 66 to 73.

66. We have

$$
Q_{0,12}=\left\{0^{2} 12\right\}+(x, y, z)^{1},
$$

where $(x, y, z)^{1}$ is a linear function of $(x, y, z)$, but depending also on the parametric points 1 and 2, which is to be determined so as to satisfy the conversion equation

$$
\partial_{1} Q_{4,12}=\partial_{4} Q_{1,45} \text {. }
$$

Observing that we have $\left\{0^{2} 23\right\}+\left\{0^{2} 31\right\}+\left\{0^{2} 12\right\}=$ a linear function of $(x, y, z)$, the linear function $(x, y, z)^{1}$ of $Q_{0,12}$ may be taken to be $=\Theta_{0,12}-\left\{0^{2} 23\right\}-\left\{0^{2} 31\right\}-\left\{0^{2} 12\right\}$; that is, we may assume

$$
\begin{aligned}
Q_{0,12} & =\left\{0^{2} 12\right\}+\Theta_{0,12}-\left(\left\{0^{2} 23\right\}+\left\{0^{2} 31\right\}+\left\{0^{2} 12\right\}\right), \\
& =-\left\{0^{2} 23\right\}-\left\{0^{2} 31\right\}+\Theta_{0,12},
\end{aligned}
$$

where $\Theta_{0,12}$ is a linear function of $(x, y, z)$, but depending also on the points 1 and 2 , which has to be determined. We have

and thence

$$
\partial_{1} Q_{0,12}=-\partial_{1}\left\{0^{2} 31\right\}+\partial_{1} \Theta_{0,12}
$$

$$
\begin{aligned}
& \partial_{1} Q_{4,12}=-\partial_{1}\left\{4^{2} 31\right\}+\partial_{1} \Theta_{4,12}, \\
& \partial_{4} Q_{1,45}=-\partial_{4}\left\{1^{2} 34\right\}+\partial_{4} \Theta_{1,45},
\end{aligned}
$$

giving an equation for $\Theta$,

$$
\partial_{1} \Theta_{4,12}-\partial_{4} \Theta_{1,45}=\partial_{1}\left\{4^{2} 31\right\}-\partial_{4}\left\{1^{2} 34\right\} ;
$$

here 4 is an arbitrary point of the quartic, and we may instead of it write 0 , the equation thus becoming

$$
\partial_{1} \Theta_{0,12}-\partial_{0} \Theta_{1,05}=\partial_{1}\left\{0^{2} 31\right\}-\partial_{0}\left\{1^{2} 30\right\} .
$$


67. Of the terms on the left-hand side, the first is a linear function of $(x, y, z)$, or say it is an integral function $0^{1}$, and the second is a linear function of $\left(x_{1}, y_{1}, z_{1}\right)$, or say it is an integral function $1^{1}$ : the given function on the right-hand side must therefore admit of expression in the form $\phi\left(0^{1}, 1,3\right)-\phi\left(1^{1}, 0,3\right)$, where $\phi\left(0^{1}, 1,3\right)$ is a known function, integral and linear as regards the coordinates $(x, y, z)$ of the point 0 , but depending also on the points 1,3 ; and $\phi\left(1^{1}, 0,3\right)$ is the like known function, integral and linear as regards the coordinates $\left(x_{1}, y_{1}, z_{1}\right)$ of the point 1 , but depending also on the points 0,3 . Moreover, since 2 and 5 are arbitrary points entering only on the left-hand side, it is clear that $\partial_{1} \Theta_{0,12}$ must be independent of 2 , and $\partial_{0} \Theta_{1,05}$ independent of 5 ; reverting to the cubic case, observe that here $\Theta_{0,12}=\int_{2}^{1} d \omega \partial_{3}\{036\}$, whence $\partial_{1} \Theta_{0,12}=\partial_{3}\{136\}$, and so $\partial_{0} \Theta_{1,05}=\partial_{3}\{036\}$, and that the corresponding equation thus is $\partial_{3}\{136\}-\partial_{3}\{036\}=\partial_{1}\{031\}-\partial_{0}\{130\}$, where the left-hand side is $=\partial_{3}\{013\}$, and the equation itself $\left(\partial_{0}+\partial_{3}+\partial_{1}\right)\{031\}=0$. We then have

$$
\partial_{1} \Theta_{0,12}-\phi\left(0^{1}, 1,3\right)=\partial_{0} \Theta_{1,02}-\phi\left(1^{1}, 0,3\right)
$$

where the one side is derived from the other by the interchange of the 0,1 . The solution therefore is

$$
\partial_{1} \Theta_{0,12}-\phi\left(0^{1}, 1,3\right)=X(\overline{0,1}, 3),
$$

a function which is symmetrical in regard to the points 0 and 1 , and, inasmuch as the left-hand is an integral function $0^{1}$, must itself be an integral function $\left(0^{1}, 1^{1}\right)$, that is, integral and linear as regards the coordinates $(x, y, z)$ and $\left(x_{1}, y_{1}, z_{1}\right)$ of the points 0 and 1 respectively. We thus have

and thence

$$
\partial_{1} \Theta_{0,12}=\phi\left(0^{1}, 1,3\right)+X(\overline{0,1}, 3)
$$

$$
\partial_{2} \Theta_{0,12}=-\phi\left(0^{1}, 2,3\right)-X(\overline{0,2}, 3)
$$

viz. the second of these expressions is, with its sign reversed, the same function of 2 that the first is of 1 .

68. It follows that, taking a new symbol 7 for the variable of the definite integral (in the cubic case $\Theta_{0,12}$ was independent of 0 , and there was nothing to prevent the use of 0 for the current point of the definite integral), we may write $\Theta_{0,12}=\int_{2}^{1} d \omega_{7} P(7,0,3)$, where $\partial_{1} P(1,0,3)=\phi\left(0^{1}, 1,3\right)+X(\overline{0,1}, 3)$, an equation which implies $\partial_{2} P(0,2,3)=\phi\left(0^{1}, 2,3\right)+X(\overline{0,2}, 3)$. But the first of these equations in $P$ is nothing else than the first of the equations in $\Theta_{0,12}$.

69. I have succeeded in finding $\phi\left(0^{1}, 1,3\right)$, but the calculation is a very tedious one, and I give only the principal steps, omitting all details. We have to bring $\partial_{1} 0^{2} 13-\partial_{0} 1^{2} 03$ into the form $\phi\left(0^{1}, 1,3\right)-\phi\left(1^{1}, 0,3\right)$. From the value of

$$
\left\{0^{2} 13\right\},=\frac{-01^{3} \cdot 03^{3}+01^{2} 3 \cdot 013^{2}-0^{2} 13 \cdot 1^{2} 3^{2}}{013 \cdot 1^{2} 3^{2}},
$$

C. XII. 
we find, by a process such as that of No. 62,

$$
\begin{aligned}
& \partial_{1}\left\{0^{2} 13\right\}=\frac{1}{\sigma^{2}}\left\{-0^{2} 3^{2} \cdot 01^{3}-0^{3} 3 \cdot j\right. \\
& +\frac{1}{q}\left(\begin{array}{c}
2.01^{3}\left[03^{3} \cdot 01^{2} 3-\left(013^{2}\right)^{2}\right] \\
\quad+\left[2.0^{2} 13.013^{2}+1.0^{2} 3^{2} .01^{23}-3.0^{2} 1^{2} \cdot 0^{2} 3^{2}\right] j
\end{array}\right) \\
& \left.+\frac{1}{q^{2}}\left(\begin{array}{r}
2.01^{3}\left(-01^{3} \cdot 03^{3}+013^{2} \cdot 01^{23}\right) g \\
-2.013^{2}\left(-01^{3} \cdot 03^{3}+013^{2} \cdot 01^{23}\right) j
\end{array}\right)\right\} \text {. }
\end{aligned}
$$

Substituting herein the values $01^{3}=\frac{1}{\Delta}(h \sigma+j \tau)$, \&c., we have $\frac{1}{\sigma^{2}}$ multiplied by a cubic function $(\rho, \sigma, \tau)^{3}$; writing down first the integral terms, and then the others, we have

$$
\begin{aligned}
\partial_{1}\left\{0^{2} 13\right\}= & \frac{1}{\Delta^{3}}\left\{\rho\left[(-6 h n+5 j m)+\frac{1}{q}\left(4 g h l-3 g j r-2 h i j+j^{2} p+2 j n l\right)+\frac{1}{q^{2}}\left(-2 g^{2} h^{2}+4 g h j n-2 j^{2} n^{2}\right)\right]\right. \\
& +\sigma\left[f j-h p+\frac{1}{q}\left(2 h i l-2 h n^{2}-3 i j r+j l p+2 j m n\right)+\frac{1}{q^{2}}\left(2 g h l n-2 g h^{2} i+2 h i j n-2 j l n^{2}\right)\right] \\
& \left.+\tau\left[3 j p+\frac{1}{q}\left(-2 g h n+2 g j m-2 i j l-2 j n^{2}\right)+\frac{1}{q^{2}}\left(2 g^{2} h l-2 g h \ddot{j}-2 g j l n+2 i j^{2} n\right)\right]\right\}
\end{aligned}
$$

(say this linear function of $\rho, \sigma, \tau$ is $=\square$ )

$$
+\frac{1}{\Delta^{3} \sigma^{2}}\left\{\rho^{3} \cdot 2 j^{2}+\rho^{2} \sigma(6 j l-3 h g)+\rho^{2} \tau \cdot 3 j q+\rho \sigma \tau(-2 g h+6 j n)+\rho \tau^{2} \cdot 2 g j+\sigma \tau^{2} \cdot 2 i j\right\} .
$$

70. The expression of $\partial_{0}\left\{1^{2} 03\right\}$ is deduced from this by the interchange of 0,1 : and I write

$$
\begin{aligned}
+\frac{1}{\sigma^{2} \Delta^{3} \rho^{3}}[ & \rho^{3}\left\{\rho^{3} \cdot 2 j^{2}+\rho^{2} \sigma(-3 h q+6 j l)+\rho^{2} \tau .3 j q+\rho \sigma \tau(-2 g h+6 j n)+\rho \tau^{2} \cdot 2 g j+\sigma \tau^{2} \cdot 2 i j\right\} \\
& -\Delta^{3}\left\{\Delta^{3} \cdot 2\left(0^{2} 3^{2}\right)^{2}-\Delta^{2} \sigma\left(-3.0^{3} 2.0^{2} 3^{2}+6.0^{3} 3 \cdot 0^{2} 23\right)-\Delta^{2} \tau .3 .0^{3} 3.0^{2} 3^{2}\right. \\
& \left.\left.+\Delta \sigma \tau\left(-2.03^{3} \cdot 0^{3} 2+6.0^{3} 3.023^{2}\right)+\Delta \tau^{2} \cdot 2.03^{3} \cdot 0^{3} 3-\sigma \tau^{2} \cdot 2 i .0^{3} 3\right\}\right],
\end{aligned}
$$

where, and in what follows, the * denotes the function immediately to the left of it, interchanging therein the 0,1 . It will be observed that the $\square$, qua linear function of $(\rho, \sigma, \tau)$, that is, of $(x, y, z)$, is a term of the required function $\phi\left(0^{1}, 1,3\right)$ : the remaining portion has to be reduced by means of the expressions for $\Delta^{2}\left(0^{2} 3^{2}\right)$, \&c., in terms of $\rho, \sigma, \tau$.

71. We obtain

$$
\begin{aligned}
& \partial_{1}\left\{0^{2} 13\right\}-\partial_{0}\left\{1^{2} 03\right\}=\square-* \\
& +\frac{1}{\Delta^{3}}\{\sigma(2 f j-3 h p-3 k q+18 l m-9 n r)+\tau(-3 g r-3 j p+9 m q)\} \\
& +\frac{1}{\Delta^{3} \rho}\left\{\sigma^{2}\left(12 f l-12 k n+18 m^{2}-9 p r\right)+\sigma \tau(6 f g-6 g k-6 i r+18 m n)+\tau^{2} \cdot 6 g m\right\} \\
& +\frac{1}{\Delta^{3} \rho^{2}}\left\{\sigma^{3}(18 f m-9 k p)+\sigma^{2} \tau(12 f n-8 i k+9 m p)+\sigma \tau^{2}(4 f g+6 i m)\right\} \\
& +\frac{1}{\Delta^{3} \rho^{3}}\left\{\sigma^{4} \cdot 4 f^{2}+\sigma^{3} \tau .6 f p+\sigma^{2} \tau^{2} \cdot 4 f i\right\}
\end{aligned}
$$


The terms of the second line may be transformed as follows:

$$
\begin{aligned}
& \frac{\sigma}{\Delta^{3}}(2 f j-3 h p-3 k q+18 l m-9 n r) \\
& =\frac{1}{2} \frac{\sigma}{\Delta^{3}}(2 f j-3 h p-3 k q+18 l m-9 n r)-* \\
& +\frac{1}{\Delta^{3} \rho}\left\{\sigma^{2}\left(-12 f l+12 k n-18 m^{2}+9 p r\right)+\sigma \tau\left(-\frac{3}{2} f q+3 g k+\frac{9}{2} i r-\frac{9}{2} l p-9 m n\right)\right\} \\
& +\frac{1}{\Delta^{3} \rho^{2}}\left\{\sigma^{3}(-30 f m+15 k p)+\sigma^{2} \tau(-12 f n+12 i k-18 m p)+\sigma \tau^{2} \cdot-9 n p\right\} \\
& +\frac{1}{\Delta^{3} \rho^{3}}\left\{\sigma^{4} \cdot-10 f^{2}+\sigma^{3} \tau \cdot-15 f p+\sigma^{2} \tau^{2} \cdot-9 p^{2}+\sigma \tau^{3} \cdot-3 i p\right\}
\end{aligned}
$$

and

$$
\begin{aligned}
& \frac{\tau}{\Delta^{3}}(-3 g r-3 j p+9 m q) \\
& =\frac{1}{2} \frac{\tau}{\Delta^{3}}(-3 g r-3 j p+9 m q)-* \\
& +\frac{1}{\Delta^{3} \rho}\left\{\sigma \tau\left(-\frac{9}{2} f q+3 g k+\frac{3}{2} i r+\frac{9}{2} l p-9 m n\right)+\tau^{2} \cdot-6 g m\right\} \\
& +\frac{1}{\Delta^{3} \rho^{2}}\left\{\sigma^{2} \tau(-9 f n+3 i k)+\sigma \tau^{2}(-6 f g-6 i m)+\tau^{3} \cdot-3 g p\right\} \\
& +\frac{1}{\Delta^{3} \rho^{3}}\left\{\sigma^{3} \tau \cdot-3 f p+\sigma^{2} \tau^{2} \cdot-6 f i+\sigma \tau^{3} \cdot-3 i p\right\}
\end{aligned}
$$

substituting these values, the whole third line is destroyed, and we find

$$
\begin{aligned}
& \partial_{1}\left\{0^{2} 13\right\}-\partial_{0}\left\{1^{2} 03\right\}=\square-* \\
& +\frac{1}{2} \cdot \frac{1}{\Delta^{3}}\{\sigma(2 f j-3 h q-3 k j+18 l m-9 n r)+\tau(-3 g r-3 j p+9 m q)\}-* \\
& +\frac{1}{\Delta^{3} p^{2}}\left\{\sigma^{3}(-12 f m+6 k p)+\sigma^{2} \tau(-9 f n+7 i k-9 m p)+\sigma \tau^{2}(-2 f g-9 n p)+\tau^{3} \cdot-3 g p\right\} \\
& +\frac{1}{\Delta^{3} \rho^{3}}\left\{\sigma^{4} \cdot-6 f^{2}+\sigma^{3} \tau \cdot-12 f p+\sigma^{2} \tau^{2}\left(-2 f i-9 p^{2}\right)+\sigma \tau^{3} \cdot-6 i p\right\} .
\end{aligned}
$$

Ultimately the last two lines of this expression are found to be

$$
\begin{aligned}
=\frac{1}{\Delta^{3}}\left\{\rho\left(-2 h n+4 j m+2 l^{2}-2 q r\right)+\sigma(-2 f j\right. & +2 h p+2 k q-10 l m+5 n r) \\
& +\tau(2 g r+h i-j p+7 \ln -4 m q)\}-*
\end{aligned}
$$

so that the whole is now a sum of three linear functions of $(\rho, \sigma, \tau) .-*$. 
72. Collecting the terms, we have

$$
\begin{gathered}
\partial_{1}\left\{0^{2} 13\right\}-\partial_{0}\left\{1^{2} 03\right\}= \\
\begin{aligned}
\frac{1}{\Delta^{3}}\left[\rho \left\{\left(-8 h n+9 j m+2 l^{2}-2 q r\right)+\frac{1}{q}\left(4 g h l-3 g j r-2 h i j+j^{2} p+2 j l n\right)\right.\right. \\
\left.+\frac{1}{q^{2}}\left(-2 g^{2} h^{2}+4 g h j n-2 j^{2} n^{2}\right)\right\}
\end{aligned} \\
+\sigma\left\{\frac{1}{2}(-h p+k q-2 l m+n r)+\frac{1}{q}\left(2 h i l-2 h n^{2}-3 \ddot{j} r+j l p+2 j m n\right)\right. \\
\left.+\frac{1}{q^{2}}\left(-2 g h^{2} i+2 g h l n+2 h i j n-2 j l n^{2}\right)\right\} \\
+\tau\left\{\frac{1}{2}(g r+2 h i+j p+m q+14 l n)+\frac{1}{q}\left(-2 g h n+2 g j m-2 i j l-2 j n^{2}\right)\right. \\
\left.\left.+* . \frac{1}{q^{2}}\left(2 g^{2} h l-2 g h i j-2 g j l n+2 i j^{2} n\right)\right\}\right]
\end{gathered}
$$

73. The right-hand side depends on the points $0,1,3$ and 2 : viz. we have therein $\rho=023, \Delta=123$, \&c., but the left-hand side depending on only the points 0,1 and 3 , the right-hand side cannot really contain 2, and it must thus remain unaltered, if for 2 we substitute any other point on the quartic, say 6 : the right-hand side may therefore be understood as a function of $0,1,3$ and 6 , viz. $\rho, \Delta, f$, \&c., will mean $063,163,633$, \&c.: we have thus $\phi\left(0^{1}, 1,3\right)=$ the above linear function with 2 thus replaced by 6 ; say

$$
\phi\left(0^{1}, 1,3\right)=\frac{1}{\Delta^{3}}[\rho()+\sigma()+\tau()]
$$

a given function of the points $0,1,3$ and the arbitrary point 6 , on the quartic curve; we therefore write it $\phi\left(0^{1}, 1,3,6\right)$. There is no obvious value for $X \overline{(0,1,3) \text { which }}$ will produce any simplification: I therefore, take this function to be $=0$; and the final result is

$$
Q_{0,12}=\left\{0^{2} 12\right\}+\Theta_{0,12}-\left(\left\{0^{2} 23\right\}+\left\{0^{2} 31\right\}+\left\{0^{2} 12\right\}\right),
$$

where $\Theta_{0,12}$ is a function integral and linear as regards the coordinates $(x, y, z)$ of the point 0 , but transcendental as regards the parametric points 1,2 ; and containing besides the arbitrary points 3,6 , of the quartic curve, its value being determined by the differential formulæ

$$
\partial_{1} \Theta_{0,12}=\phi\left(0^{1}, 1,3,6\right), \quad \partial_{2} \Theta_{0,12}=-\phi\left(0^{1},-2,3,6\right),
$$

where $\phi\left(0^{1}, 1,3,6\right)$ is a given function as above. I do not see the meaning of the very complicated linear function of $(\rho, \sigma, \tau)$, nor how to reduce it to any form such as the simple one $\partial_{3}\{036\}$, which presents itself in the case of the cubic curve.

Cambridge, England, October 5, 1882. 


\section{Chapter IV. The Major Function $(x, y, z)_{12}{ }^{n-2}$, continued.}

The Conversion, Fixed Curve a Quartic, continued. Art. Nos. 74 to 82 .

74. I resume the question considered ante Nos. 66 to 73 . The general problem, where the fixed curve is any given curve whatever, has recently been solved in a very complete and elegant form by $\mathrm{Dr}$ Nöther, in the two notes "Zur Reduction algebraischer Differentialausdrücke auf die Normalformen" and "Ueber die algebraischen Differentialausdrücke, 2 ${ }^{\mathrm{e}}$ Note," Sitzungsb. der phys.-med. Soc. zu Erlangen, 10 Dec. 1883 and 14 Jan. 1884. I consider here the case of the quartic curve, $n=4$, and connect his result with my former investigations.

We have the differential

$$
\frac{(x, y, z)_{12}{ }^{n-2} d \omega}{012},=\frac{\Omega_{12} d \omega}{012}
$$

where $\Omega_{12}$, or as $I$ also write it $\Omega(0 ; 1,2 ; 3,4,5)$, is a rational and integral function of the degree $(n-2=) 2$ in the current coordinates $(x, y, z)$ : it depends also on the parametric points 1,2 , which are points on the quartic, coordinates $\left(x_{1}, y_{1}, z_{1}\right)$, $\left(x_{2}, y_{2}, z_{2}\right)$ respectively; and on $(p=) 3$ other points $3,4,5$ on the quartic, coordinates $\left(x_{3}, y_{3}, z_{3}\right),\left(x_{4}, y_{4}, z_{4}\right),\left(x_{5}, y_{5}, z_{5}\right)$ respectively. The curve $\Omega=0$ is a conic, which is taken to pass through the dps (none in the present case) and through the $(n-2=) 2$ residues of the parametric points; and the function $\Omega$ is such that on writing therein $\left(x_{1}, y_{1}, z_{1}\right)$ for $(x, y, z)$ it becomes $=\left(n .1^{n-1} 2^{*}=\right)_{4} .1^{32}:$ viz. we have $\Omega(1 ; 1,2 ; 3,4,5)={ }_{4} .1^{32}$, which implies also $\Omega(2 ; 1,2 ; 3,4,5)={ }_{4} \cdot 12^{3}$ : so defined, the function would contain $(p=) 3$ arbitrary constants, but these are determined so that the curve $\Omega=0$ passes through the 3 points $3,4,5$ on the quartic: and the function $\Omega,=\Omega(0: 1,2: 3,4,5)$ is thus a completely determinate function, rational and integral of the degree 2 in the coordinates $(x, y, z)$ of the current point, and rational in the coordinates of the other five points respectively. I call to mind that 012 denotes the determinant formed with the coordinates $(x, y, z)$, \&c., of the points $0,1,2$ respectively: the like notation is used throughout.

75. The function $\Omega(0 ; 1,2 ; 3,4,5)$ is, in fact, the function $\Omega^{\prime}$ of No. 43 with only the further condition in regard to the points $3,4,5$ of the quartic; viz. $\Omega$ is the function determined by the equation

$$
\begin{aligned}
& \left|\begin{array}{ccc}
(x, y, z)^{2} & , & \Omega \\
1\left(x_{1}, y_{1}, z_{1}\right)^{2} & , & 4.1^{32} \\
2\left(x_{1}, y_{1}, z_{1} \gamma x_{2},\right. & y_{2}, & \left.z_{2}\right) \\
1\left(x_{2}, y_{2}, z_{2}\right)^{2} & 6.1^{22^{2}} \\
\left(x_{3}, y_{3}, z_{3}\right)^{2} & , & 4.12^{3} \\
\left(x_{4}, y_{4}, z_{4}\right)^{2} & , & 0 \\
\left(x_{5}, y_{5}, z_{5}\right)^{2} & , & 0
\end{array}\right|=0: \\
& *\left(x_{2} \frac{d}{d x_{1}}+y_{2} \frac{d}{d y_{1}}+z_{2} \frac{d}{d z_{1}}\right) f\left(x_{1}, y_{1}, z_{1}\right)=n .1^{n-1} 2 \text {; see No. } 2 .
\end{aligned}
$$


this is of the form

$$
M \Omega+\square=0,
$$

and as appears in No. 46, $M$ is $=123.124 .125 .345$. Hence writing $\square(0 ; 1,2 ; 3,4,5)$ for $\square$, we have

Hence further writing

$$
\Omega,=\Omega(0 ; 1,2 ; 3,4,5),=\frac{-\square(0 ; 1,2 ; 3,4,5)}{123.124 .125 .345}
$$

$$
Q,=Q(0 ; 1,2 ; 3,4,5),=\frac{\Omega(0 ; 1,2 ; 3,4,5)}{012}
$$

so that the differential is $Q d \omega,=Q(0 ; 1,2 ; 3,4,5) d \omega$, we have

$$
Q=Q(0 ; 1,2 ; 3,4,5),=\frac{-\square(0 ; 1,2 ; 3,4,5)}{012.123 .124 .125 .345}
$$

which is of the form

$$
Q=\frac{0^{2} \overline{12} \sqrt{345^{2}}}{0^{1} \overline{12} \overline{2}^{4} \overline{345^{2}}},=0^{1} \overline{12345^{0}},
$$

viz. $Q$ is a rational fraction where the numerator is of the degree 2 , and the denominator of the degree 1 as regards the coordinates $(x, y, z)$ of the current point: but the numerator and denominator are each of the degree 4 as regards the coordinates of the points 1, 2 separately, and of the degree 2 as regards the coordinates of the points 3, 4, 5 separately: that is, $Q$ is of the degree 1 as regards the coordinates $(x, y, z)$, but of the degree 0 as regards the coordinates of the points $1,2,3,4,5$ separately.

76. The signification of the symbol of quasi-differentiation $\partial$ (applicable only to a function of the degree 0 in the coordinates to which the differentiations have reference) is explained ante No. 60. The function $Q$ just mentioned is of the degree 0 in regard to the coordinates of each of the points 1, 2, 3, 4, 5; and it can thus be operated upon by the symbols $\partial_{1}, \partial_{2}, \partial_{3}, \partial_{4}, \partial_{5}$ respectively. Observe, in particular, that we have $\partial_{1} Q(0 ; 1,2 ; 3,4,5)=\overline{01}^{1} \overline{2345^{0}}$, viz. it is of the degree 1 in the coordinates of the points 0 and 1 respectively, but of the degree 0 in regard to the coordinates of the points $2,3,4,5$ respectively.

77. This being so, we may consider the function

$$
\begin{aligned}
H(0 ; 1,2 ; 3,4,5 ; 6,7,8) & =\partial_{1} Q(0 ; 1,2 ; 3,4,5) \\
& +\partial_{3} Q(1 ; 3,2 ; 6,4,5) \cdot \frac{045}{345} \\
& +\partial_{4} Q(1 ; 4,2 ; 3,7,5) \cdot \frac{053}{453} \\
& +\partial_{5} Q(1 ; 5,2 ; 3,4,8) \cdot \frac{034}{534}
\end{aligned}
$$

where $6,7,8$ are arbitrary points on the quartic; the functions

$$
\partial_{3} Q(1 ; 3,2 ; 6,4,5), \quad \partial_{4} Q(1 ; 4,2 ; 3,7,5), \quad \partial_{5} Q(1 ; 5,2 ; 3,4,8),
$$


are functions of the same form as $\partial_{1} Q(0 ; 1,2 ; 3,4,5)$, and derived from it by changing in each case the current point 0 into the parametric point 1 , and by further changing in the three cases this parametric point into the points $3,4,5$ respectively, and replacing the eorresponding point 3,4 or 5 by the new arbitrary point 6,7 or 8 . Further 045 , \&c., denote determinants as above; so that in $H$ each of the last three terms is, in fact, as regards the point 0 , a mere linear function of the coordinates $(x, y, z)$ of this point.

We have $\partial_{3} Q(1 ; 3,2 ; 6,4,5)=\overline{13}^{1} \overline{2645}^{\circ}$, and hence this function multiplied by $\frac{045}{345}$ is $=\overline{01}^{123456^{\circ}}$; and so for the third and fourth terms of $H$ : thus each of the four terms of $H$ is $=\overline{01}^{12345678^{\circ}}$, of the degree 1 in the coordinates of the points 0 and 1 respectively, but of the degree 0 in the coordinates of the other points $2,3,4,5,6,7,8$ respectively.

Nöther's conversion-theorem consists herein, that the function

$$
H(0 ; 1,2 ; 3,4,5 ; 6,7,8)
$$

is unaltered by the interchange of the two points 0,1 ; or putting for shortness

$$
H(0 ; 1,2 ; 3,4,5 ; 6,7,8)=H_{1}(0),
$$

the theorem is

$$
H_{1}(0)=H_{0}(1)
$$

78. We have, No. 59,

$$
\frac{\frac{1}{2} \Omega_{12}}{012}=\frac{-01^{3} \cdot 02^{3}+01^{22} \cdot 012^{2}+0^{2} 12 \cdot 1^{2} 2^{2}}{012 \cdot 1^{2} 2^{2}},=\left\{0^{2} 12\right\}
$$

or as for greater simplicity I write it $=0^{2} 12$,

viz. $0^{2} 12$ is now written instead of $\left\{0^{2} 12\right\}$ to denote the function just given as the value of $\frac{\frac{1}{2} \Omega_{12}}{012}, \Omega_{12}$ is thus $=2.012 .0^{2} 12$, viz. this is a particular form of $\Omega_{12}$ satisfying the conditions that $\Omega_{12}=0$ is a conic passing through the residues of the points 1,2 , and such that $\Omega_{12}$ on writing therein $\left(x_{1}, y_{1}, z_{1}\right)$ for $(x, y, z)$ becomes $={ }_{4} .1^{32}$ : hence the general form of the function satisfying these conditions is $=2.012\left\{0^{2} 12+\right.$ arbitrary linear function of $(x, y, z)\}$. The before-mentioned function $\Omega(0 ; 1,2 ; 3,4,5)$ is a function satisfying these conditions and the further conditions that the conic $\Omega=0$ shall pass through the three points $3,4,5$ on the quartic: these further conditions serve to determine the linear function: and we at once obtain

$$
\frac{\frac{1}{2} \Omega(0 ; 1,2 ; 3,4,5)}{012}=0^{2} 12-3^{2} 12 \frac{045}{345}-4^{2} 12 \frac{053}{453}-5^{2} 12 . \frac{034}{534},
$$

viz. the value of $\Omega$ given by this equation, on writing therein $0=3$, 4 , or 5 , becomes $=0$ as it should do. 
79. We thus have

$$
Q(0 ; 1,2 ; 3,4,5)=0^{2} 12-3^{2} 12 \frac{045}{345}-4^{2} 12 \frac{053}{453}-5^{2} 12 \frac{034}{534}
$$

and Nöther's conversion-equation becomes

$$
\begin{aligned}
& \partial_{1}\left\{0^{2} 12-3^{2} 12 \frac{045}{345}-4^{2} 12 \frac{053}{453}-5^{2} 12 \frac{034}{534}\right\} \\
+ & \partial_{3}\left\{1^{232}-6^{2} 32 \frac{145}{645}-4^{2} 32 \frac{156}{456}-5^{232} \frac{164}{564}\right\} \cdot \frac{045}{345} \\
+ & \partial_{4}\left\{1^{2} 42-3^{2} 42 \frac{175}{375}-7^{2} 42 \frac{153}{753}-5^{2} 42 \frac{137}{537}\right\} \cdot \frac{053}{453} \\
+ & \partial_{5}\left\{1^{252}-3^{2} 52 \frac{148}{348}-4^{2} 52 \frac{183}{483}-5^{2} 48 \frac{134}{834}\right\} \cdot \frac{034}{534} \\
= & \partial_{0}\left\{1^{2} 02-3^{2} 02 \frac{145}{345}-4^{2} 02 \frac{153}{453}-5^{2} 02 \frac{134}{534}\right\} \\
+ & \partial_{3}\left\{0^{2} 32-6^{2} 32 \frac{045}{645}-4^{2} 32 \frac{056}{456}-5^{2} 32 \frac{064}{564}\right\} \cdot \frac{145}{345} \\
+ & \partial_{4}\left\{0^{2} 42-3^{2} 42 \frac{075}{375}-7^{2} 42 \frac{053}{753}-5^{2} 42 \frac{037}{537}\right\} \cdot \frac{153}{453} \\
+ & \partial_{5}\left\{0^{2} 52-3^{2} 52 \frac{048}{348}-4^{2} 52 \frac{083}{483}-5^{2} 48 \frac{034}{834}\right\} \cdot \frac{134}{534}
\end{aligned}
$$

an equation where the functions operated on with the $\partial$ 's are only functions such as $0^{2} 12$; for there is not any determinant operated upon containing the number which is the suffix of the $\partial$ operating upon it.

80. Taking all the terms over to the left-hand side, there are in all 32 terms: but of these $3+3$ destroy each other, and $6+6$ unite in pairs into 6 terms: there are thus in all $7+7+6,=20$ terms: viz. multiplying the whole equation by 345 , it is found that the equation becomes

$$
\begin{aligned}
& \text { or as this may } \\
& \text { be written where }
\end{aligned}
$$

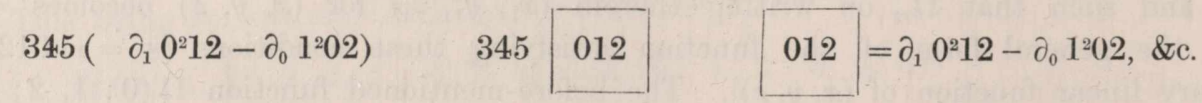

$$
\begin{aligned}
& -045\left(-\partial_{3} 1^{2} 32+\partial_{1} 3^{2} 12\right) \quad-045 \quad 312 \\
& -053\left(-\partial_{4} 1^{2} 42+\partial_{1} 4^{2} 12\right) \quad-305412
\end{aligned}
$$

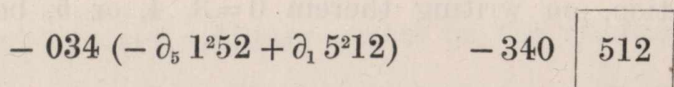




$$
\begin{array}{ll}
-145\left(\partial_{3} 0^{2} 32-\partial_{0} 3^{2} 02\right) & -145032 \\
-153\left(\partial_{4} 0^{2} 42-\partial_{0} 4^{2} 02\right) & -315 \\
-134\left(\partial_{5} 0^{2} 52-\partial_{0} 5^{2} 02\right) & -341 \\
+013\left(-\partial_{4} 5^{2} 42+\partial_{5} 4^{2} 52\right) & +301 \\
+0142 \\
+014\left(-\partial_{5} 3^{2} 52+\partial_{3} 5^{2} 32\right) & +140 \\
+015\left(-\partial_{3} 4^{2} 32+\partial_{4} 3^{2} 42\right) & +015 \\
\hline 0 & =0,
\end{array}
$$

viz. the equation is

$$
\Sigma \pm 345012=0
$$

the nine terms which follow the first term 345012 of the sum being obtained by the interchanges of 0,1 (one or each) with the $3,4,5$, each interchange giving rise to a sign -.

81. In obtaining the foregoing result, we have, for instance, a pair of terms

$$
\partial_{4} 5^{2} 42 \frac{-137.053+037.153}{537},=\partial_{4} 5^{2} 42 \frac{-013.537}{537},=\partial_{4} 5^{2} 42(-013)
$$

viz. this depends on the equation

$$
137.053-037.153-537.013=0 \text {, }
$$

or say

$$
-137.035+037.135+013.357=0 \text {, }
$$

an identity which, in a form which will be readily understood, may be written

$$
\operatorname{det}\left|\begin{array}{l}
0137 \\
013735
\end{array}\right|=0 .
$$

Similarly, the two terms which contain $\partial_{5} 4^{2} 52$ combine into the single term $\partial_{5} 4^{2} 52(013)$ : and the two new terms taken together are

$$
013\left(-\partial_{4} 5^{2} 42+\partial_{5} 4^{2} 52\right) \cdot=301452 .
$$

C. XII. 
82. The proof of the identity,

$$
\Sigma \pm 345012=0
$$

depends on the property of the function

$$
012,=\partial_{1} 0^{2} 12-\partial_{0} 1^{2} 02
$$

enunciated No. 67, and proved $\grave{a}$ posteriori by the tedious calculation Nos. 69 to 73 , viz. in No. 67, writing 2 in place of 3 , this is: $-\partial_{1} 0^{2} 12-\partial_{0} 1^{2} 02$ is equal to the difference of two functions, the first of them linear in the coordinates $(x, y, z)$ of the point 0 , but depending also on the coordinates of the points 1 and 2 ; the second of them linear in the coordinates $\left(x_{1}, y_{1}, z_{1}\right)$ of the point 1 but depending also on the coordinates of the points 0 and 2 . Or, what is the same thing, the property is

$$
012=A_{12} x+B_{12} y+C_{12} z-\left(A_{02} x_{1}+B_{02} y_{1}+C_{02} z_{1}\right),
$$

where $A_{12}, B_{12}, C_{12}$ are functions of $\left(x_{1}, y_{1}, z_{1}\right),\left(x_{2}, y_{2}, z_{2}\right)$, and $A_{02}, B_{02}, C_{02}$ are the like functions of $(x, y, z),\left(x_{2}, y_{2}, z_{2}\right)$.

Substituting such values in the sum $\Sigma \pm 345|012|$, but writing down only the terms which contain $x$, these are

$$
\begin{aligned}
& 345\left(A_{12} x-A_{02} x_{1}\right) \\
& -045\left(A_{12} x_{3}-A_{32} x_{1}\right) \quad-145\left(A_{32} x-A_{02} x_{3}\right) \quad+301\left(A_{52} x_{4}-A_{42} x_{5}\right) \\
& -305\left(A_{12} x_{4}-A_{42} x_{1}\right) \quad-315\left(A_{42} x-A_{02} x_{4}\right) \quad+140\left(A_{32} x_{5}-A_{52} x_{3}\right) \\
& -340\left(A_{12} x_{5}-A_{52} x_{1}\right) \quad-341\left(A_{52} x-A_{02} x_{5}\right) \quad+015\left(A_{42} x_{3}-A_{32} x_{4}\right) .
\end{aligned}
$$

This is

$$
\begin{aligned}
= & A_{02}\left(-x_{1} 345+x_{3} 145+x_{4} 315+x_{5} 341\right) \\
+ & A_{12}\left(\quad x 345-x_{3} 045-x_{4} 305-x_{5} 340\right) \\
+ & A_{32}\left(\quad x_{1} 045-x 145+x_{5} 140-x_{4} 015\right) \\
+ & A_{42}\left(-x 315+x_{3} 015+x_{1} 305-x_{5} 301\right) \\
+ & A_{52}\left(-x 341+x_{4} 301+x_{1} 340-x_{3} 140\right)
\end{aligned}
$$

where the coefficient of each of the $A$ 's is identically $=0$ : and similarly, the terms in $y$ and the terms in $z$ are each $=0$. We have thus the proof of the identity

$$
\Sigma \pm 3450012=0
$$

that is, of the conversion-equation $H_{1}(0)=H_{0}(1)$. 
The Syzygy-Fixed Curve a Quartic. Art. No. 83.

I revert to the theory of the Syzygy, ante No. 59.

83. We have

$$
Q(0 ; 1,2 ; 3,4,5)=0^{2} 12-3^{2} 12 \frac{045}{345}-4^{2} 12 \frac{053}{453}-5^{2} 12 \frac{034}{534},
$$

or if for convenience we take instead of 1,2 , the parametric points to be $\alpha, \beta$ coordinates $\left(x_{\alpha}, y_{\alpha}, z_{\alpha}\right)$ and $\left(x_{\beta}, y_{\beta}, z_{\beta}\right)$ respectively, then this equation is

$$
Q_{\alpha \beta}=Q(0 ; \alpha, \beta ; 3,4,5)=0^{2} \alpha \beta-3^{2} \alpha \beta \frac{045}{345}-4^{2} \alpha \beta \frac{053}{453}-5^{2} \alpha \beta \frac{034}{534} .
$$

Considering a new parametric point $\gamma$, and forming the like functions $Q_{\beta \gamma}$ and $Q_{\gamma \alpha}$, it is to be shown that we have identically

$$
Q_{\alpha \beta}+Q_{\beta \gamma}+Q_{\gamma \alpha}=0 .
$$

To prove this, observe that, in the equation at the end of No. 59, $\Delta, \rho, \sigma, \tau$ denote $123,023,031,012$ respectively. Hence writing therein $\alpha, \beta, \gamma$ in place of $1,2,3$ respectively, and putting $A, B, C$ for the coefficients (including therein the factor $\frac{1}{\Delta^{2}}$ ) of $\rho, \sigma, \tau$ respectively, the equation is

$$
0^{2} \beta \gamma+0^{2} \gamma \alpha+0^{2} \alpha \beta=A .0 \beta \gamma+B .0 \gamma \alpha+C .0 \alpha \beta,
$$

where $A, B, C$ are absolute constants (functions, that is; of the coefficients of the quartic) each divided by $(\alpha \beta \gamma)^{2}$. We hence obtain

$$
\begin{aligned}
\left(Q_{\beta \gamma}+Q_{\gamma \alpha}+Q_{\alpha \beta}\right) .345= & 345(A .0 \beta \gamma+B .0 \gamma \alpha+C .0 \alpha \beta) \\
- & 045(A .3 \beta \gamma+B .3 \gamma \alpha+C .3 \alpha \beta) \\
& -053(A .4 \beta \gamma+B .4 \gamma \alpha+C .4 \alpha \beta) \\
& -034(A .5 \beta \gamma+B .5 \gamma \alpha+C .5 \alpha \beta) .
\end{aligned}
$$

On the left-hand side the whole coefficient of $A$ is $=0$; viz. the coefficient has the value det. $\left|\begin{array}{l}0345 \\ 0345 \beta \gamma\end{array}\right|$, which is =0. Similarly, the whole coefficient of $B$ is $=0$, and the whole coefficient of $C$ is $=0$ : and we have thus the required result

$$
Q_{\beta \gamma}+Q_{\gamma \alpha}+Q_{\alpha \beta}=0
$$

The syzygy is thus obtained in a more perfect form than in No. 59 ; viz. by considering (instead of $0^{2} \alpha \beta$ ) the new form $Q_{\alpha \beta}$, then, instead of a sum which is a linear function of the coordinates $(x, y, z)$, we obtain a sum $=0$. 
The Fixed Curve a Cubic-Syzygy and Conversion. Art. Nos. 84 and 85.

84. In the case when the fixed curve is a cubic (see Nos. 58 and 64), the analogous formulæ are

$$
\frac{1}{3} \Omega(0 ; 1,2 ; 3)=\frac{1^{22} .023+12^{2} \cdot 031}{123},=\widetilde{012}-\frac{\widetilde{123}}{123} 012(\text { see No. } 49),
$$

that is,

$$
Q(0 ; 1,2 ; 3),=\frac{\frac{1}{3} \Omega}{012},=\frac{\widetilde{012}}{012}-\frac{\widetilde{123}}{123},=\{012\}-\{123\}
$$

where 1, 2 are the parametric points: 3 any other point on the cubic: the brackets \{\} are of course here necessary in order to distinguish $\{012\}$ from the determinant 012. It will be remembered that $\{012\}$ is an alternate function

$$
\{012\},=-\{102\},=\{120\}, \& \mathrm{c} .
$$

If instead of 1,2 we take the parametric points to be $\alpha, \beta$, coordinates $\left(x_{a}, y_{a}, z_{a}\right)$ and $\left(x_{\beta}, y_{\beta}, z_{\beta}\right)$ respectively, then the formula is

$$
Q_{\alpha \beta}=Q(0 ; \alpha, \beta ; 3)=\{0 \alpha \beta\}-\{3 \alpha \beta\} .
$$

Hence taking on the cubic a new point $\gamma$, coordinates $\left(x_{\gamma}, y_{\gamma}, z_{\gamma}\right)$ and forming the functions $Q_{\beta \gamma}$ and $Q_{\gamma \alpha}$ we have

$$
\begin{aligned}
Q_{\beta \gamma}+Q_{\gamma \alpha}+Q_{\alpha \beta}= & \{0 \beta \gamma\}+\{0 \gamma \alpha\}+\{0 \alpha \beta\} \\
& -\{3 \beta \gamma\}-\{3 \gamma \alpha\}-\{3 \alpha \beta\} .
\end{aligned}
$$

But by the formula No. 58,

hence also

$$
\{0 \beta \gamma\}+\{0 \gamma \alpha\}+\{0 \alpha \beta\}=\{\alpha \beta \gamma\}
$$

and we have thus

$$
\{3 \beta \gamma\}+\{3 \gamma \alpha\}+\{3 \alpha \beta\}=\{\alpha \beta \gamma\}:
$$

the syzygy for the cubic.

$$
Q_{\beta \gamma}+Q_{\gamma \alpha}+Q_{\alpha \beta}=0
$$

85. For the conversion, the definition of $H$ is

$$
\begin{aligned}
H(0 ; 1,2 ; 3,6)= & \partial_{1} Q(0 ; 1,2 ; 3) \\
& +\partial_{3} Q(1 ; 3,2 ; 6),
\end{aligned}
$$

viz. this is

$$
\begin{aligned}
H_{0}(1)=H(0 ; 1,2 ; 3,6)= & \partial_{1}(\{012\}-\{123\}) \\
& +\partial_{3}(\{132\}-\{326\}), \\
= & \partial_{1}\{012\}-\left(\partial_{1}+\partial_{3}\right)\{123\}-\partial_{3}\{326\},
\end{aligned}
$$

which, in virtue of $\left(\partial_{1}+\partial_{2}+\partial_{3}\right)\{123\}=0$ (see No. 63), becomes

$$
H_{0}(1)=\partial_{1}\{012\}+\partial_{2}\{123\}-\partial_{3}\{326\} \text {. }
$$


Interchanging the 0 and 1 , we thence have

$$
H_{1}(0)=\partial_{0}\{102\}+\partial_{2}\{023\}-\partial_{3}\{326\} .
$$

Hence the difference $H_{0}(1)-H_{1}(0)$ is

viz. this is

$$
=\partial_{1}\{012\}-\partial_{0}\{102\}+\partial_{2}\{123\}-\partial_{2}\{023\},
$$

where the first term is

$$
=\left(\partial_{1}+\partial_{0}\right)\{012\}+\partial_{2}(\{123\}-\{230\}),
$$

$$
=-\partial_{2}\{012\},
$$

and the whole therefore is

$$
\begin{aligned}
& =\partial_{2}(\{123\}-\{230\}-\{012\}) \\
& =-\partial_{2}\{301\},
\end{aligned}
$$

in virtue of

$$
\{123\}-\{230\}+\{301\}-\{012\}=0 ;
$$

the whole is consequently $=0$.

We have thus

$$
H_{0}(1)-H_{1}(0)=0,
$$

the conversion-equation in the case of the cubic.

\section{Chapter V. Miscellaneous Investigations.}

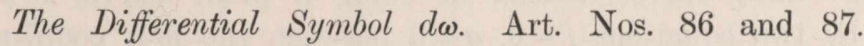

86. The definition is

$$
\frac{y d z-z d y}{\frac{d f}{d x}}=\frac{z d x-x d z}{\frac{d f}{d y}}=\frac{x d y-y d x}{\frac{d f}{d z}}=d \omega,
$$

and it hence follows that we have

$$
d \omega=\frac{\left|\begin{array}{ccc}
d x, & d y, & d z \\
x, & y, & z \\
\lambda, & \mu, & \nu
\end{array}\right|}{\lambda \frac{d f}{d x}+\mu \frac{d f}{d y}+\nu \frac{d f}{d z}}
$$

where $(\lambda, \mu, \nu)$ are arbitrary constants or, if we please, arbitrary functions of $(x, y, z)$ : viz. the expression just written down is altogether independent of the values of $\lambda, \mu, \nu$ : and is consequently equal to the value obtained by writing any two of these symbols $=0$, that is, the expression is equal to any one of the foregoing three equal values of $d \omega$. The expression was first given by Aronhold (1863), in the memoir presently referred to. 
It is to be remarked that, considering $(\lambda, \mu, \nu)$ as the coordinates of a point, the denominator $\lambda \frac{d f}{d x}+\mu \frac{d f}{d y}+\nu \frac{d f}{d z}$ equated to 0 is the polar $(n-1)$ thic of the point $\lambda, \mu, \nu$ in regard to the fixed curve.

If instead of $\lambda, \mu, \nu$ we write $b c^{\prime}-b^{\prime} c, c a^{\prime}-c^{\prime} a, a b^{\prime}-a^{\prime} b$, where $(a, b, c)\left(a^{\prime}, b^{\prime}, c^{\prime}\right)$ are constants, then the numerator is

$$
=(a x+b y+c z)\left(a^{\prime} d x+b^{\prime} d y+c^{\prime} d z\right)-\left(a^{\prime} x+b^{\prime} y+c^{\prime} z\right)(a d x+b d y+c d z),
$$

or introducing $\rho, \sigma$ to denote the arbitrary linear functions $a x+b y+c z$ and $a^{\prime} x+b^{\prime} y+c^{\prime} z$ respectively, the numerator is $=\rho d \sigma-\sigma d \rho$ : moreover, observing that $a, b, c$ and $a^{\prime}, b^{\prime}, c^{\prime}$ are the differential coefficients of $\rho, \sigma$ in regard to the coordinates $(x, y, z)$, the denominator is $=J(f, \rho, \sigma)$; and the value of $d \omega$ is

$$
d \omega=\frac{\rho d \sigma-\sigma d \rho}{J(f, \rho, \sigma)}
$$

where, in accordance with a previous remark, the denominator equated to 0 is the polar $(n-1)$ thic of the intersection of the lines $\rho=0, \sigma=0$ in regard to the fixed curve.

Obviously, by taking for $\rho, \sigma$ any two of the three coordinates $x, y, z$, we reproduce the original three forms of $d \omega$.

87. The last-mentioned form of $d \omega$ suggests the expression for this symbol in the case where the fixed curve, instead of being a plane curve, is a curve of double curvature defined by two equations $f=0, g=0$ between the four coordinates $(x, y, z, w)$ : viz. $\rho, \sigma$ being now arbitrary linear functions

$$
a x+b y+c z+d w, \text { and } a^{\prime} x+b^{\prime} y+c^{\prime} z+d^{\prime} w
$$

of the four coordinates, the expression is

$$
d \omega=\frac{\rho d \sigma-\sigma d \rho}{J(f, g, \rho, \sigma)}:
$$

and by taking for $\rho, \sigma$ any two of the four coordinates $x, y, z, w$, we have for $d \omega$ six values which must of course be equal to each other; it is easy to verify $\grave{a}$ posteriori that this is so.

In the case where the curve of double curvature is not the complete intersection of two surfaces, the denominator (regarded as the Jacobian of the curve and of the arbitrary planes $\rho, \sigma$ ) will have a definite meaning, but what this is $\mathbf{I}$ do not at present consider.

The last-mentioned expression for $d \omega$ will be applied further on to the case of the quadri-quadric curve $y^{2}+x^{2}=1, z^{2}+k^{2} x^{2}=1$. 
Integral Formula. Art. Nos. 88 to 90 .

88. In what precedes, $d \omega$ has been used as a single symbol to denote any one of the equal differential expressions

$$
\frac{y d z-z d y}{\frac{d f}{d x}},=\frac{z d x-x d z}{\frac{d f}{d y}},=\frac{x d y-y d x}{\frac{d f}{d z}}
$$

there is no quantity $\omega$. The expressions are of the order $-(n-3)$ in the coordinates $(x, y, z)$, and since $(x, y, z)$ are as to their absolute magnitudes altogether arbitrary (only their ratios being determinate), a symbol such as

$$
\omega,=\int d \omega,=\int \frac{y d z-z d y}{\frac{d f}{d y}},
$$

would, except in the case $n=3$, be altogether meaningless. In fact, the integral would be

$$
\int \frac{z^{2} d\left(\frac{y}{z}\right)}{z^{n-1} \phi\left(\frac{x}{z}, \frac{y}{z}\right)},=\int \frac{d\left(\frac{y}{z}\right)}{z^{n-s} \phi\left(\frac{x}{z}, \frac{y}{z}\right)}
$$

where $\frac{x}{z}$ is, by the equation of the fixed curve, given as a function of $\frac{y}{z}$; but the other factor $z^{n-3}$ is an absolutely indeterminate variable value, and the expression is meaningless.

But we have integrals $\int Q d \omega$, where $Q$ is a homogeneous function of the order $n-3$ in the coordinates $(x, y, z)$; and, in particular, we have such integrals where (corresponding to the forms which present themselves in the differential pure and affected theorems respectively) $Q$ is either a rational and integral function $(x, y, z)^{n-3}$, or a rational and integral function $(x, y, z)^{n-2}$ divided by a linear function $(x, y, z)^{1}$ : for in every such case, the form of integral is

$$
\int \frac{d\left(\frac{y}{z}\right)}{\phi\left(\frac{x}{z}, \frac{y}{z}\right)}
$$

where $\frac{x}{z}$ is a given function of $\frac{y}{z}$, and the factor of $d\left(\frac{y}{z}\right)$ is thus a mere function of $\frac{y}{z}$, More definitely, in the integrals $\int Q d \omega$ which are considered, $Q$ is either a minor function $(x, y, z)^{n-3}$, or it is the quotient of a major function $(x, y, z)_{12}{ }^{n-2}$ by the linear function 012. 
In the case $n=2$, there is no rational and integral function $(x, y, z)^{n-3}$, but the function may be of the form belonging to the affected theorem, viz. it is unity divided by a linear function $(x, y, z)^{1}$; or say the integral is $\int \frac{d \omega}{\alpha x+\beta y+\gamma z}$, where the $(x, y, z)$ are connected by a quadric equation $(a, \ldots \gamma x, y, z)^{2}=0$ : it will be shown presently that this integral is obtainable as a logarithmic function.

In the case $n=3$, we have the rational and integral function $(x, y, z)^{n-3}$, = a constant, or say $=1$, so that there is here an integral $\int d \omega$ : we do not call this $\omega$, but introducing a new letter, say $u$, and fixing at pleasure the inferior limit of the integral, we write $u=\int d \omega$.

89. In the foregoing form $\int Q d \omega$, so long as we retain the symbol $d \omega$, there is nothing to show what is the variable in regard to which the integration is to be performed; we may, for instance, writing

$$
d \omega=\frac{y^{2} d\left(\frac{z}{y}\right)}{\frac{d f}{d x}}
$$

make it to be $\frac{y}{z}$, or in like manner to be any other of the six quotients. We thus cannot attribute a value to the inferior or superior limit of such an integral, but we may take the limits to be each of them a point on the fixed curve: for instance, if 1,0 be points on the fixed curve, then the integral $\int_{0}^{1} Q d \omega$ means the integral taken from the value at the point 0 to the value at the point 1 of the variable in regard to which the integration is performed; or when there is no expressed superior limit, then the integral is to be taken from the value for the expressed or known inferior limit to the value at the current point $(x, y, z)$ of the variable in regard to which the integration is performed. The actual value of the integral will of course depend upon the path of the variable; but this is a question which is not here entered upon.

If using Cartesian Coordinates $x, y$, we write for instance

$$
d \omega=\frac{d x}{\frac{d f}{d y}}, \text { then } \int \frac{Q d x}{\frac{d f}{d y}}
$$

will denote an integral $\int \phi x d x$ in regard to the variable $x$, and the inferior and superior limits will be as usual values of $x$; or if there is no expressed superior limit, then the integral $\int_{0} \phi x d x$ will be the integral taken from the inferior limit $x_{0}$ to the current value $x$. 
We may, if we please, consider the coordinates $(x, y, z)$ as depending upon a parameter 8 , viz. the ratios $x: y: z$ may be regarded as given functions of 8 , and the integral $\int Q d \omega$, is then an integral $\int \Omega d z$, which taken from a constant inferior limit up to the value 8 , belonging to a given point 1 of the curve, is a given function of $8_{1}$, or say of the point 1. But except in the case of the cubic (or generally if $p=1$ ), we do not have the coordinates actually given as known functions of a parameter \& (say they are potentially known functions of \&), and it is further to be noticed the functions which present themselves are functions not of a single point, but of $p$ or more points: thus in the case of the quartic, $n=4, p=3$; we have $\int^{1} x d \omega, \int^{1} y d \omega, \int^{1} z d \omega$, each standing for a given function of the parameter $z_{1}$, but these integrals do not present themselves singly, but in combinations such as

$$
\left(\int^{1}+\int^{2}+\int^{3}+\int^{\xi}\right)(x d \omega, y d \omega, z d \omega)
$$

say these sums of integrals are $u, v, w$ : each of the functions $u, v, w$ is a potentially known function of the parameters $\varangle_{1}, 8_{2}, 8_{3}, \gamma_{\xi}$ which belong to the points $1,2,3, \xi$ respectively, and is consequently regarded as a given function of these four points.

90. Consider as before, in the case of a cubic curve, the integral $u=\int d \omega$ : it will presently be seen that for the general curve as given by a cubic equation $f=0$ of any form whatever, we arrive at a form of elliptic function: but the ordinary elliptic functions sn, cn, dn connect themselves most readily with the cubic curve $y^{2}=x .1-x .1-k^{2} x$. We have here

$$
d \omega=\frac{\frac{1}{2} d x}{y},=\frac{\frac{1}{2} d x}{\sqrt{x .1-x .1-k^{2} x}},
$$

or, in the equation $u=\int d \omega$, taking the inferior limit to be 0 , say

$$
u=\int_{0} \frac{\frac{1}{2} d x}{\sqrt{x .1-x \cdot 1-k^{2} x}}
$$

an equation which determines $u$ as a function of $x$, or conversely, $x$ as a function of $u$. We might thence, by means of Abel's theorem as applied to the curve in question, investigate the properties of the function $x=\lambda(u)$ thus arising, and so establish the theory of elliptic functions: but it is more convenient, treating the elliptic functions as known functions, to write for $\lambda u$ its value; viz. to take for $x$ as given by this equation, the value $x=\operatorname{sn}^{2} u$ : we thence have $y=\operatorname{sn} u \operatorname{cn} u \operatorname{dn} u$; viz. these values $x=\operatorname{sn}^{2} u, y=\operatorname{sn} u \operatorname{cn} u \operatorname{dn} u$, satisfy the equation $y^{2}=x .1-x .1-k^{2} x$ of the curve, and give, moreover, $d \omega=d u=\frac{\frac{1}{2} d x}{y}$ : and we can with these values, and the formulæ for elliptic functions, verify any results given by Abel's theorem. This will be done in considerable detail : but at present I wish only to remark that the formulæ give the coordinates $x, y$ of a point on the cubic curve expressed as one-valued functions of c. XII. 
a parameter or argument $u$ : but that this argument $u$ is not a one-valued function of the coordinate $x$, or even of the coordinates $x, y$ of the given point on the curve: say the argument $u$ has not a unique value for a given point $(x, y)$ of the curve. There are, in fact, an infinity of values $u=u_{0}+2 m K+2 m^{\prime} i K^{\prime}$, where $m, m^{\prime}$ are any positive or negative integers: that this is so, depends on the multiplicity of values, according to the different paths of the variable, of the integral

$$
u=\int_{0} \frac{\frac{1}{2} d x}{\sqrt{x .1-x \cdot 1-k^{2} x}}
$$

or, regarding the elliptic functions as known functions, it depends upon the double periodicity of these functions.

Aronhold's Quadric Integral. Art. Nos. 91 to 93.

91. I reproduce the investigation contained in Aronhold's paper "Ueber eine neue algebraische Behandlungsweise u.s.w." Crelle, t. LXI. (1863), pp. 95-145. We take $f$ the general quadric function $\left(a, b, c, f, g, h \gamma(x, y, z)^{2} ; \alpha x+\beta y+\gamma z\right.$ an arbitrary linear function of $x, y, z:$ the theorem is $\frac{d \omega}{\alpha x+\beta y+\gamma z}=$ differential of logarithm of an algebraic function of $(x, y, z)$; viz. taking $(\xi, \eta, \zeta)$ for the coordinates of either of the points of intersection of the line $\alpha x+\beta y+\gamma z=0$ with the quadric $(a, \ldots\rangle(x, y, z)^{2}=0$, and writing also

$$
\Omega^{2}=-\left(b c-f^{2}, c a-g^{2}, a b-h^{2}, g h-a f, h f-b g, f g-\operatorname{ch} \gamma \alpha, \beta, \gamma\right)^{2},
$$

then the theorem is

$$
\frac{d \omega}{\alpha x+\beta y+\gamma z}=\frac{1}{\Omega} d \cdot \log \frac{(a, \ldots \gamma x, y, z \gamma \xi, \eta, \zeta)}{\alpha x+\beta y+\gamma z},
$$

or, what is the same thing,

$$
\int \frac{d \omega}{\alpha x+\beta y+\gamma z}=\frac{1}{\Omega} \log \frac{(a, \ldots \gamma x, y, z \gamma \xi, \eta, \zeta)}{\alpha x+\beta y+\gamma z}+\text { const. }
$$

It is to be observed, in reference to this equation, that the two sides respectively are in regard to $(\alpha, \beta, \gamma)$ homogeneous functions of the degree -1 , and in regard to $(\xi, \eta, \zeta)$ homogeneous of the degree 0 ; viz. on the right-hand side the effect of a change in the absolute magnitudes of $\xi, \eta$, $\zeta$, say the change into $k \xi, k \eta, k \zeta$, is merely to change by $\log k$ the constant of integration.

It is to be remarked also that the equation $(a, \ldots \gamma \xi, \eta, \zeta \gamma x, y, z)=0$ represents the tangent to the conic at the point $(\xi, \eta, \zeta)$ of intersection with the line $\alpha x+\beta y+\gamma z=0$; calling the linear function in question $T$, the value of the integral is $\frac{1}{\Omega} \log \frac{T}{\alpha x+\beta y+\gamma z}$; if $\left(\xi_{1}, \eta_{1}, \zeta_{1}\right),\left(\xi_{2}, \eta_{2}, \zeta_{2}\right)$ are the coordinates of the two points of intersection respectively, then in passing from one of these to the other we change the sign of the radical $\Omega$, and the two values thus are $\frac{1}{\Omega} \log \frac{T_{1}}{\alpha x+\beta y+\gamma z}$ and $-\frac{1}{\Omega} \log \frac{T_{2}}{\alpha x+\beta y+\gamma z}$. These must 
differ by a constant only; viz. we should have $\log \frac{T_{1} T_{2}}{(\alpha x+\beta y+\gamma z)^{2}}=$ a const. And, in fact, $T_{1}$ and $T_{2}$ being the tangents to the conic $f$ at its intersections with the line $\alpha x+\beta y+\gamma z=0$, we have it is clear $f=\lambda T_{1} T_{2}+\mu(\alpha x+\beta y+\gamma z)^{2}$, that is, $(x, y, z)$ referring to a point of the conic $f=0$, we have $\frac{T_{1} T_{2}}{(\alpha x+\beta y+\gamma z)^{2}}=$ a constant, which is right.

92. We require the coordinates $(\xi, \eta, \zeta)$ of an intersection: these are determined by the equations $\alpha \xi+\beta \eta+\gamma \zeta=0,(a, \ldots \gamma \xi, \eta, \zeta)^{2}=0$, or as these may be written

$$
\begin{gathered}
\alpha \xi+\beta \eta \quad+\gamma \zeta=0 \\
(\alpha \xi+h \eta+g \zeta) \xi+(h \xi+b \eta+f \zeta) \eta+(g \xi+f \eta+c \zeta) \zeta=0
\end{gathered}
$$

we have thence $\xi, \eta, \zeta$ proportional to the determinants

$$
\left|\begin{array}{rrr}
a \xi+h \eta+g \zeta, & h \xi+b \eta+f \zeta, & g \xi+f \eta+c \zeta \\
\alpha, & \beta \quad, & \gamma
\end{array}\right|
$$

say these determinants are $\Omega \xi, \Omega \eta, \Omega \zeta$, where $\Omega$ is a value as yet undetermined. The equations are $\gamma(h \xi+b \eta+f \zeta)-\beta(g \xi+f \eta+c \zeta)-\Omega \xi=0$, \&c., viz. these are

$$
\begin{array}{lll}
(\gamma h-\beta g-\Omega) \xi+(\gamma b-\beta f & ) \eta+(\gamma f-\beta c & ) \zeta=0 \\
(\alpha g-\gamma a & ) \xi+(\alpha f-\gamma h-\Omega) \eta+(\alpha c-\gamma g & ) \zeta=0 \\
(\beta a-\alpha h & ) \xi+(\beta h-\alpha b & ) \eta+(\beta g-\alpha f-\Omega) \zeta=0
\end{array}
$$

eliminating $(\xi, \eta, \zeta)$, we have an equation which may be written

$$
\left|\begin{array}{lll}
A, & B, & C \\
A^{\prime}, & B^{\prime}, & C^{\prime} \\
A^{\prime \prime}, & B^{\prime \prime}, & C^{\prime \prime}
\end{array}\right|-\Omega\left(B^{\prime} C^{\prime \prime}-B^{\prime \prime} C^{\prime}+C^{\prime \prime} A-C A^{\prime \prime}+A B^{\prime}-A^{\prime} B\right)+\Omega^{2}\left(A+B^{\prime}+C^{\prime \prime}\right)-\Omega^{3}=0
$$

that is,

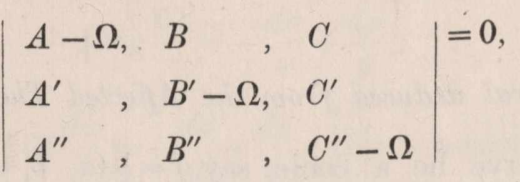

We find very easily that the determinant and $A+B^{\prime}+C^{\prime \prime}$ are each $=0$; and the equation thus reduces itself to

$$
\Omega^{2}=B^{\prime} C^{\prime \prime}-B^{\prime \prime} C^{\prime}+C^{\prime \prime} A-C A^{\prime \prime}+A B^{\prime}-A^{\prime} B,
$$

or substituting for $A, B$, \&c., their values,

$$
\Omega^{2}=-\left(b c-f^{2}, \ldots \gamma \alpha, \beta, \gamma\right)^{2}
$$

this being so, the ratios of $\xi, \eta, \zeta$ are determined by means of any two of the foregoing linear equations. 
93. We may now verify the theorem; in the general expression for $d \omega$ writing for $\lambda, \mu, \nu$ the values $\xi, \eta, \zeta$, the equation to be verified becomes

$\frac{\left|\begin{array}{lll}d x, & d y, & d z \\ x, & y, & z \\ \xi, & \eta, & \zeta\end{array}\right|}{(\alpha x+\beta y+\gamma z)(a, \ldots \gamma x, y, z \gamma \xi, \eta, \zeta)}=\frac{1}{\Omega}\left\{\frac{(a, \ldots \gamma \xi, \eta, \zeta \gamma d x, d y, d z)}{(a, \ldots \gamma \xi, \eta, \zeta \gamma \quad x, y, z)}-\frac{\alpha d x+\beta d y+\gamma d z}{\alpha x+\beta y+\gamma z}\right\}$,

viz. this is

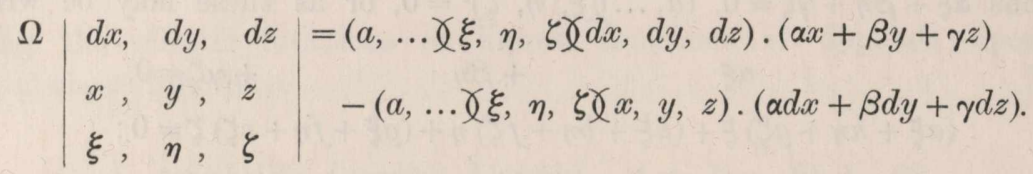

Here, on the right-hand side, the coefficient of $d x$ is

which is

$$
\begin{gathered}
(a \xi+h \eta+g \zeta)(\alpha x+\beta y+\gamma z) \\
-\alpha\{(a \xi+h \eta+g \zeta) x+(h \xi+b \eta+f \zeta) y+(g \xi+f \eta+c \zeta) z\},
\end{gathered}
$$

$$
\begin{aligned}
= & y\{\beta(a \xi+h \eta+g \zeta)-\alpha(h \xi+b \eta+f \zeta)\} \\
& -z\{\alpha(g \xi+f \eta+c \zeta)-\gamma(a \xi+h \eta+g \zeta)\} \\
= & y \cdot \Omega \zeta-z \cdot \Omega \eta \\
= & \Omega(y \zeta-z \eta)
\end{aligned}
$$

which is right; and similarly, the coefficients of $d y$ and $d z$ have the same values on the two sides of the equation respectively.

Aronhold's Quadric Integral deduced from the Affected Theorem. Art. Nos. 94 to 98.

94. Let the fixed curve be a conic, say $f=\frac{1}{2}\left(a, b, c, f, g, h \gamma(x, y, z)^{2},=0\right.$ : and let the variable curve be a line meeting the conic in the points 3 and 4 . The affected theorem is

$$
\Sigma \frac{12 d \omega}{012}=-\frac{\delta 134}{134}+\frac{\delta 234}{234}
$$

where $\left(x_{1}, y_{1}, z_{1}\right)$ and $\left(x_{2}, y_{2}, z_{2}\right)$ being the coordinates of the points 1 and 2 respectively, 12 denotes the constant $\left(a, \ldots \gamma\left(x_{1}, y_{1}, z_{1} \gamma\left(x_{2}, y_{2}, z_{2}\right)\right.\right.$ : and 012 , \&c., denote determinants as usual.

The left-hand side is here

$$
12\left\{\frac{d \omega_{3}}{312}+\frac{d \omega_{4}}{412}\right\}:
$$

on the right-hand side, $\delta$ refers to the variation of the constants of $\phi$, that is, to the variations of the points 3 and 4 ; or we may write $\delta=d_{3}+d_{4}$; the points 3,4 are independent, and the equation, being satisfied at all, must be satisfied separately 
in regard to the variations of 3 , and in regard to the variations of 4 : we must therefore have

$$
12 \frac{d \omega_{3}}{312}=-\frac{d_{3} 134}{134}+\frac{d_{3} 234}{234}
$$

and the like equation obtained herefrom by the interchange of the numbers 3 and 4 .

95. The equation just written down relates to any four points $1,2,3,4$ of the conic; and if for 3,4 we write 0,3 respectively, it becomes

$$
12 \frac{d \omega}{012}=-\frac{d .031}{031}+\frac{d .023}{023}
$$

which relates to the points $0,1,2,3$ of the conic: writing, as before, $023,031,012=\rho, \sigma, \tau$, this equation is

$$
12 \frac{d \omega}{\tau}=-\frac{d \sigma}{\sigma}+\frac{d \rho}{\rho}
$$

which may be verified as follows : the equation of the conic is $f=23 . \sigma \tau+31 \cdot \tau \rho+12 . \rho \sigma,=0$ : we have $d \omega=\frac{\rho d \sigma-\sigma d \rho}{\frac{d f}{d \tau}}$, where $\frac{d f}{d \tau}=23 \cdot \sigma+31 \rho,=-\frac{12 \cdot \rho \sigma}{\tau}$, that is, $d \omega=\frac{12}{\tau}\left(-\frac{d \sigma}{\sigma}+\frac{d \rho}{\rho}\right)$, the equation in question.

96. We have, as a property of any four points $0,1,2,3$ of a conic,

$$
\frac{23}{123.023}=\frac{-01}{012.031} \text {, or say } \frac{23}{\Delta \cdot \rho}=\frac{-01}{\sigma \tau} \text {, that is, } \frac{23}{\Delta} \frac{\sigma}{\rho}=-\frac{01}{\tau} \text {; }
$$

hence considering 0 as a variable point, and differentiating the logarithms,

$$
-d \log \frac{01}{\tau}=-\frac{d \sigma}{\sigma}+\frac{d \rho}{\rho}
$$

and the foregoing equation $12 \frac{d \omega}{\tau}=-\frac{d \sigma}{\sigma}+\frac{d \rho}{\rho}$ thus becomes $12 \frac{d \omega}{\tau}=-d \log \frac{01}{\tau}$, or restoring for $\tau$ its value 012 ,

$$
12 \frac{d \omega}{012}=-d \log \frac{01}{012} \text {. }
$$

Taking now $\alpha x+\beta y+\gamma z=0$ for the equation of the line 012 ; this meets the conic in the points 1,2 , coordinates $\left(x_{1}, y_{1}, z_{1}\right)$ and $\left(x_{2}, y_{2}, z_{2}\right)$ respectively: and we have

and from this last value

$$
\begin{aligned}
\alpha, \beta, \gamma & =y_{1} z_{2}-y_{2} z_{1}, z_{1} x_{2}-z_{2} x_{1}, x_{2} y_{1}-x_{1} y_{2}, \\
12 & \left.=(a, \ldots) x_{1}, y_{1}, z_{1} \backslash x_{2}, y_{2}, z_{2}\right),
\end{aligned}
$$

$$
\overline{12}^{2}=\left\{\left(a, \ldots \chi x_{1}, y_{1}, z_{1} \chi x_{2}, y_{2}, z_{2}\right)\right\}^{2}-\left(a, \ldots \chi x_{1}, y_{1}, z_{1}\right)^{2} \cdot\left(a, \ldots \chi x_{2}, y_{2}, z_{2}\right)^{2}
$$

(the second term being of course $=0$ ), viz. this is

$$
\begin{aligned}
\overline{12}^{2} & =-\left(b c-f^{2}, \ldots \gamma\left(y_{1} z_{2}-y_{2} z_{1}, z_{1} x_{2}-z_{2} x_{1}, x_{1} y_{2}-x_{2} y_{1}\right)^{2}\right. \\
& =-\left(b c-f^{2}, \ldots \gamma(\alpha, \beta, \gamma)^{2},\right.
\end{aligned}
$$


or say $12=-\Omega$, if $\Omega^{2}=-\left(b c-f^{2}, \ldots \gamma \alpha, \beta, \gamma\right)^{2}$ as before: and the equation thus is

$$
\frac{\Omega d \omega}{\alpha x+\beta y+\gamma z}=d \log \frac{\left(\alpha, \ldots \gamma x_{1}, y_{1}, z_{1} \gamma(x, y, z)\right.}{\alpha x+\beta y+\gamma z},
$$

or finally, writing $(\xi, \eta, \zeta)$ instead of $\left(x_{1}, y_{1}, z_{1}\right)$ to denote the coordinates of one or other of the intersections of the line $\alpha x+\beta y+\gamma z=0$ with the conic, the equation becomes

$$
\frac{\Omega d \omega}{\alpha x+\beta y+\gamma z}=d \log \frac{(a, \ldots \gamma \xi, \eta, \zeta \gamma(x, y, z)}{\alpha x+\beta y+\gamma z},
$$

which is Aronhold's quadric integral.

97. (The foregoing property, which may also be written

$$
\frac{23}{023.123}=\frac{01}{201.301}
$$

is verified very simply in the case of four points $0,1,2,3$ of a circle: in fact

$$
\begin{aligned}
23 & =x_{2} x_{3}+y_{2} y_{3}-1,=\cos 23-1,=-2 \sin ^{2} \frac{1}{2} 23, \\
023 & =2 \sin \frac{1}{2} 23 \sin \frac{1}{2} 30 \sin \frac{1}{2} 02 ;
\end{aligned}
$$

and so for the other like expressions; each side of the equation is thus reduced to $1 \div \sin \frac{1}{2} 02 \sin \frac{1}{2} 03 \sin \frac{1}{2} 12 \sin \frac{1}{2} 13$.)

98. In particular, if the conic is taken to be the circle $x^{2}+y^{2}-1=0$, then for the coordinates $\left(\frac{\xi}{\zeta}, \frac{\eta}{\zeta}\right)$ of the intersections with the line $\alpha x+\beta y+\gamma=0$, we have

giving $\Omega^{2}=\alpha^{2}+\beta^{2}+\gamma^{2}$; and then

$$
\begin{gathered}
\Omega \xi+\gamma \eta+\beta \zeta=0 \\
\gamma \xi-\Omega \eta+\alpha \zeta=0 \\
\beta \xi-\alpha \eta+\Omega \zeta=0
\end{gathered}
$$

$$
\begin{aligned}
\xi: \eta: \zeta & =-\beta^{2}+\gamma^{2}: \alpha \beta-\gamma \Omega: \quad \alpha \gamma+\beta \Omega \\
& =-\alpha \beta-\gamma \Omega: \alpha^{2}-\gamma^{2}: \beta \gamma+\alpha \Omega \\
& =\alpha \gamma+\beta \Omega: \beta \gamma-\alpha \Omega:-\alpha^{2}-\beta^{2}
\end{aligned}
$$

The formula then becomes

$$
\int \frac{d x}{\left(\alpha x+\beta \sqrt{1-x^{2}}+\gamma\right) \sqrt{1-x^{2}}}=\frac{1}{\sqrt{\alpha^{2}+\beta^{2}-\gamma^{2}}} \log \frac{\xi x+\eta \sqrt{1-x^{2}}-\zeta}{\alpha x+\beta \sqrt{1-x^{2}}+\gamma}:
$$

or, retaining $\Omega, y$ for the values $\sqrt{\alpha^{2}+\beta^{2}-\gamma^{2}}$, and $\sqrt{1-x^{2}}$, this may also be written

$$
=\frac{1}{\Omega} \log \frac{\gamma(\alpha x+\beta y+\gamma)+\Omega(\beta x-\alpha y+\Omega)}{\alpha x+\beta y+\gamma} .
$$

The form of the integral is still such that the value is not very readily obtainable by ordinary methods: the value just written down can of course be verified, but the verification is scarcely easier than for the original more general form. 
In the very particular case $\alpha=0, \beta=0, \gamma=1$, we have $\Omega=i ; \xi: \eta: \zeta=1:-i: 0$, and the formula becomes

viz. this is

$$
\int \frac{d x}{\sqrt{1-x^{2}}}=\frac{1}{i} \log (x-i y) \text { : }
$$

$$
\sin ^{-1} x=\frac{1}{2} \pi+\frac{1}{i} \log \left(x-i \sqrt{1-x^{2}}\right)
$$

which is right; for putting $\sin ^{-1} x=u$, and therefore $x=\sin u$, the equation becomes $i\left(u-\frac{1}{2} \pi\right)=\log (\sin u-i \cos u)$ : that is, $\cos \left(u-\frac{1}{2} \pi\right)+i \sin \left(u-\frac{1}{2} \pi\right)=\sin u-i \cos u$.

\section{Fixed Curve a Cubic: the Parametric Points 1, 2 consecutive points on the Curve.} Art. Nos. 99 to 106.

99. The major function $(x, y, z)_{12}^{1}$ is taken to be $=\frac{1^{22} .023-12^{2} .013}{123}$, so that, calling the differential $Q d \omega$, we have

$$
Q=\frac{122.023-12^{2} \cdot 013}{123.012}
$$

it is required to find what this becomes when 1, 2 are consecutive points on the curve, or what is the same thing when the line 012 is a tangent at the point 1.

I take for convenience the cubic to be $f,=\frac{1}{3}\left(x^{3}+y^{3}+z^{3}\right)$, $=0$. The coordinates of 1 are $\left(x_{1}, y_{1}, z_{1}\right)$, those of 2 are $\left(x_{1}+\delta x_{1}, y_{1}+\delta y_{1}, z_{1}+\delta z_{1}\right)$, or as for shortness I write them $\left(x_{1}+\alpha, y_{1}+\beta, z_{1}+\gamma\right)$, where $\alpha, \beta, \gamma$ are considered as infinitesimals of the first order: this being so, the denominator of $Q$ is at once seen to be of the second order; it will appear that the numerator is of the third order; whence $Q$ is of the first order.

100. We have

$$
d \omega=\frac{y d z-z d y}{x^{2}},=\frac{z d x-x d z}{y^{2}}=\frac{x d y-y d x}{z^{2}}
$$

and in analogy herewith we may write

this being so, we have

$$
\delta \omega_{1}=\frac{y_{1} \gamma-z_{1} \beta}{x_{1}^{2}},=\frac{z_{1} \alpha-x_{1} \gamma}{y_{1}^{2}},=\frac{x_{1} \beta-y_{1} \alpha}{z_{1}^{2}}
$$

$$
012=\left|\begin{array}{ccc}
x, & y, & z \\
x_{1} & y_{1} & z_{1} \\
\alpha & \beta & \gamma
\end{array}\right|=\left(x x_{1}^{2}+y y_{1}^{2}+z z_{1}^{2}\right) \delta \omega_{1}=01^{2} \cdot \delta \omega_{1}
$$

and similarly $312=31^{2} \cdot \delta \omega_{1}$.

Moreover

$$
023=\left|\begin{array}{lll}
x, & y, & z \\
x_{1} & y_{1} & z_{1} \\
x_{3} & y_{3} & z_{3}
\end{array}\right|+\left|\begin{array}{ccc}
x, & y, & z \\
\alpha, & \beta, & \gamma \\
x_{3}, & y_{3}, & z_{3}
\end{array}\right|=013+0 \delta 13
$$


as the second term may be written; moreover

$$
\begin{aligned}
& 1^{2} 2=x_{1}^{2}\left(x_{1}+\alpha\right)+y_{1}^{2}\left(y_{1}+\beta\right)+z_{1}^{2}\left(z_{1}+\gamma\right),=\alpha x_{1}^{2}+\beta y_{1}^{2}+\gamma z_{1}^{2}, \\
& 12^{2}=x_{1}\left(x_{1}+\alpha\right)^{2}+y_{1}\left(y_{1}+\beta\right)^{2}+z_{1}\left(z_{1}+\gamma\right)^{2},=2\left(\alpha x_{1}{ }^{2}+\beta y_{1}{ }^{2}+\gamma z_{1}^{2}\right)+\alpha^{2} x_{1}+\beta^{2} y_{1}+\gamma^{2} z_{1},
\end{aligned}
$$

and hence

or reducing by

$$
\begin{aligned}
1^{2} 2.023-12^{2} .013= & \left(\alpha x_{1}{ }^{2}+\beta y_{1}{ }^{2}+\gamma z_{1}^{2}\right) \quad(013+0 \delta 13) \\
& -\left[2\left(\alpha x_{1}{ }^{2}+\beta y_{1}{ }^{2}+\gamma z_{1}{ }^{2}\right)+\left(\alpha^{2} x_{1}+\beta^{2} y_{1}+\gamma^{2} z_{1}\right)\right] 013 \\
= & -\left[\left(\alpha x_{1}{ }^{2}+\beta y_{1}{ }^{2}+\gamma z_{1}{ }^{2}\right)+\left(\alpha^{2} x_{1}+\beta^{2} y_{1}+\gamma^{2} z_{1}\right)\right] 013 \\
& +\left(\alpha x_{1}{ }^{2}+\beta y_{1}{ }^{2}+\gamma z_{1}{ }^{2}\right) .0 \delta 13,
\end{aligned}
$$

this is

$$
3\left(\alpha x_{1}^{2}+\beta y_{1}{ }^{2}+\gamma z_{1}^{2}\right)+3\left(\alpha^{2} x+\beta^{2} y+\gamma^{2} z\right)+\left(\alpha^{3}+\beta^{3}+\gamma^{3}\right)=0,
$$

$$
=+\frac{1}{3}\left(\alpha^{3}+\beta^{3}+\gamma^{3}\right) 013-\left(\alpha^{2} x_{1}+\beta^{2} y_{1}+\gamma^{2} z_{1}\right) 0 \delta 13,
$$

which is of the third order. in fact,

101. We may show that each of the terms contains the factor $\left(\delta \omega_{1}\right)^{2}$ : we have,

$$
\begin{aligned}
& y_{1} z_{1}\left(\delta \omega_{1}\right)^{2}=\alpha \beta \frac{x_{1}}{y_{1}}-\alpha^{2}-\beta \gamma \frac{x_{1}^{2}}{y_{1} z_{1}}+\gamma \alpha \frac{x_{1}}{z_{1}}, \\
& z_{1} x_{1}\left(\delta \omega_{1}\right)^{2}=\beta \gamma \frac{y_{1}}{z_{1}}-\beta^{2}-\gamma \alpha \frac{y_{1}^{2}}{z_{1} x_{1}}+\alpha \beta \frac{y_{1}}{x_{1}}, \\
& x_{1} y_{1}\left(\delta \omega_{1}\right)^{2}=\gamma \alpha \frac{z_{1}}{x_{1}}-\gamma^{2}-\alpha \beta \frac{z_{1}^{2}}{x_{1} y_{1}}+\beta \gamma \frac{z_{1}}{y_{1}}
\end{aligned}
$$

hence, first multiplying by $\alpha, \beta, \gamma$ and adding, we have

$$
\begin{aligned}
\left(\alpha y_{1} z_{1}+\beta z_{1} x_{1}+\gamma x_{1} y_{1}\right)\left(\delta \omega_{1}\right)^{2}= & \frac{\beta \gamma}{y_{1} z_{1}}\left(\beta y_{1}^{2}+\gamma z_{1}^{2}\right)+\frac{\gamma \alpha}{z_{1} x_{1}}\left(\gamma z_{1}^{2}+\alpha x_{1}^{2}\right)+\frac{\alpha \beta}{x_{1} y_{1}}\left(\alpha x_{1}^{2}+\beta y_{1}^{2}\right) \\
& -\alpha \beta \gamma\left(\frac{x_{1}^{2}}{y_{1} z_{1}}+\frac{y_{1}^{2}}{z_{1} x_{1}}+\frac{z_{1}^{2}}{x_{1} y_{1}}\right) \\
& -\left(\alpha^{3}+\beta^{3}+\gamma^{3}\right) .
\end{aligned}
$$

But in virtue of $\alpha x_{1}{ }^{2}+\beta y_{1}{ }^{2}+\gamma z_{1}{ }^{2}=0$, the first line becomes $=$ the second line, or the two together are

$$
=-2 \times \beta \gamma\left(\frac{x_{1}^{2}}{y_{1} z_{1}}+\frac{y_{1}^{2}}{z_{1} x_{1}}+\frac{z_{1}^{2}}{x_{1} y_{1}}\right),
$$

which is $=0$ in virtue of $x_{1}^{3}+y_{1}{ }^{3}+z_{1}^{3}=0$; hence the equation is

$$
\left(\alpha y_{1} z_{1}+\beta z_{1} x_{1}+\gamma x_{1} y_{1}\right)\left(\delta \omega_{1}\right)^{2}=-\left(\alpha^{3}+\beta^{3}+\gamma^{5}\right),
$$

the required expression for the first term.

102. Again, multiplying by $x_{1}, y_{1}, z_{1}$, and adding, we have $3 x_{1} y_{1} z_{1}\left(\delta \omega_{1}\right)^{2}=\frac{\beta \gamma}{y_{1} z_{1}}\left(y_{1}{ }^{3}+z_{1}{ }^{3}-x_{1}{ }^{3}\right)+\frac{\gamma \alpha}{z_{1} x_{1}}\left(z_{1}{ }^{3}+x_{1}{ }^{3}-y_{1}{ }^{3}\right)+\frac{a \beta}{x_{1} y_{1}}\left(x_{1}{ }^{3}+y_{1}{ }^{3}-z_{1}{ }^{3}\right)-\left(\alpha^{2} x_{1}+\beta^{2} y_{1}+\gamma^{2} z_{1}\right)$ 
where, in virtue of $x_{1}{ }^{3}+y_{1}{ }^{3}+z_{1}{ }^{3}=0$, the first line is

$$
=-\frac{2}{x_{1} y_{1} z_{1}}\left(\beta \gamma x_{1}^{4}+\gamma \alpha y_{1}^{4}+\alpha \beta z_{1}^{4}\right),
$$

and this again is $=-2\left(\alpha^{2} x_{1}+\beta^{2} y_{1}+\gamma^{2} z_{1}\right)$ : in fact, we have identically

$$
\begin{aligned}
x_{1} y_{1} z_{1}\left(\alpha^{2} x_{1}+\beta^{2} y_{1}+\gamma^{2} z_{1}\right)= & \left(\alpha x_{1}{ }^{2}+\beta y_{1}{ }^{2}+\gamma z_{1}{ }^{2}\right)\left(\alpha y_{1} z_{1}+\beta z_{1} x_{1}+\gamma x_{1} y_{1}\right) \\
& -\left(\beta \gamma x_{1}+\gamma x y_{1}+\alpha \beta z_{1}\right)\left(x_{1}^{3}+y_{1}{ }^{3}+z_{1}^{3}\right) \\
& +\left(\beta \gamma x_{1}{ }^{4}+\gamma \alpha y_{1}{ }^{4}+\alpha \beta z_{1}^{4}\right),
\end{aligned}
$$

which, in virtue of $\alpha x_{1}{ }^{2}+\beta y_{1}{ }^{2}+\gamma z_{1}{ }^{2}=0$, and $x_{1}{ }^{3}+y_{1}{ }^{3}+z_{1}{ }^{3}=0$, becomes

$$
x_{1} y_{1} z_{1}\left(\alpha^{2} x_{1}+\beta^{2} y_{1}+\gamma^{2} z_{1}\right)=\left(\beta \gamma x_{1}^{4}+\gamma \alpha y_{1}^{4}+\alpha \beta z_{1}^{4}\right) \text {. }
$$

Hence the equation is

or finally

$$
\begin{aligned}
3 x_{1} y_{1} z_{1}\left(\delta \omega_{1}\right)^{2} & =-3\left(\alpha^{2} x_{1}+\beta^{2} y_{1}+\gamma^{2} z_{1}\right), \\
x_{1} y_{1} z_{1}\left(\delta \omega_{1}\right)^{2} & =-\quad\left(\alpha^{2} x_{1}+\beta^{2} y_{1}+\gamma^{2} z_{1}\right),
\end{aligned}
$$

the required expression for the second term.

103. Writing for shortness

we have

$$
\alpha y_{1} z_{1}+\beta z_{1} x_{1}+\gamma x_{1} y_{1}=\delta\left(x_{1} y_{1} z_{1}\right)
$$

$$
1^{22} .023-12^{2} .013=\left\{-\frac{1}{3} \delta\left(x_{1} y_{1} z_{1}\right) 013+x_{1} y_{1} z_{1} .0 \delta 13\right\}\left(\delta \omega_{1}\right)^{2},
$$

and hence dividing by

we have

$$
012 \cdot 123,=01^{2} \cdot 31^{2} \cdot\left(\delta \omega_{1}\right)^{2},
$$

$$
Q=\frac{1^{22} .023-12^{2} \cdot 013}{012.123}=\frac{-\frac{1}{3} \delta\left(x_{1}, y_{1}, z_{1}\right) \cdot 013+x_{1} y_{1} z_{1} \cdot 0 \delta 13}{01^{2} .31^{2}} .
$$

But this can be further reduced: the numerator, multiplied by 3 , is

which is

$$
=-\left(\alpha y_{1} z_{1}+\beta z_{1} x_{1}+\gamma x_{1} y_{1}\right)\left|\begin{array}{ccc}
x, & y, & z \\
x_{1}, & y_{1}, & z_{1} \\
x_{3}, & y_{3}, & z_{3}
\end{array}\right|+3 x_{1} y_{1} z_{1}\left|\begin{array}{ccc}
x, & y, & z \\
\alpha, & \beta, & \gamma \\
x_{3}, & y_{3}, & z_{3}
\end{array}\right| \text {, }
$$

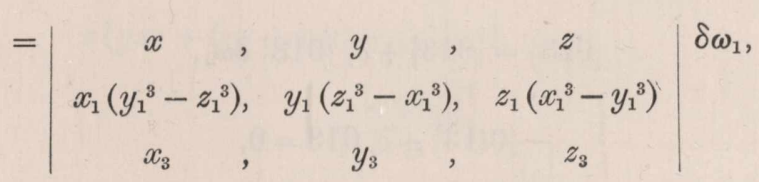

where $x_{1}\left(y_{1}^{3}-z_{1}^{3}\right), y_{1}\left(z_{1}^{3}-x_{1}^{3}\right), z_{1}\left(x_{1}^{3}-y_{1}^{3}\right)$ are the coordinates of the tangential of the point 1 in regard to the cubic, viz. the point of intersection of the tangent at 1 with the cubic. The determinant may for shortness be called $0 t 13$; and we thus have

$$
Q=\frac{1^{22} .023-12^{2} \cdot 013}{012.123}=\frac{\frac{1}{3} \delta \omega_{1}}{31^{2}} \frac{0 t 13}{01^{2}}
$$

where observe that $01^{2}=0$ is the equation of the tangent at the point $0:$ and $0 t 13=0$ is the equation of the line joining the tangential of 1 with the arbitrary point 3 .

C. XII. 
104. The identity just referred to is proved very easily. Comparing on each side the coefficient of $y z_{3}-y_{3} z$, the factor $x_{1}$ divides out and we ought to have

that is,

$$
-\left(\alpha y_{1} z_{1}+\beta z_{1} x_{1}+\gamma x_{1} y_{1}\right)+3 y_{1} z_{1} \alpha=\left(y_{1}^{3}-z_{1}^{3}\right) \delta \omega_{1}
$$

$$
\left(y_{1}^{3}-z_{1}^{3}\right) \delta \omega_{1}=2 \alpha y_{1} z_{1}-\beta z_{1} x_{1}-\gamma x_{1} y_{1}:
$$

and, in fact, from $y_{1}^{2} \delta \omega_{1}=z_{1} \alpha-x_{1} \gamma, z_{1}^{2} \delta \omega_{1}=x_{1} \beta-y_{1} \alpha$, we have

$$
\left(y_{1}^{3}-z_{1}^{3}\right) \delta \omega_{1}=y_{1}\left(z_{1} \alpha-x_{1} \gamma\right)-z_{1}\left(x_{1} \beta-y_{1} \alpha\right),
$$

which is the value in question. Similarly the coefficients of $z x_{3}-z_{3} x, x y_{3}-x_{3} y$ are equal on the two sides; and the equation is thus verified.

105. The proof has been given in regard to the particular cubic $x^{3}+y^{3}+z^{3}=0$; but it might have been given for the canonical form $x^{3}+y^{3}+z^{3}+6 l x y z=0$ : and from the invariantive form it is clear that the result in fact applies to any cubic whatever. The result is an important one: we see by it that when the points 1 and 2 are consecutive points on the curve we must, in place of the differential $Q d \omega$, which is evanescent, consider a new form $\frac{0 t 13}{01^{2}} \delta \omega_{1}$, where, as already remarked, the denominator represents the tangent at the point 1 , and the numerator the line joining the tangential of this point with the point 3 .

106. We have

$$
\{023\}+\{031\}+\{012\}=\{123\}
$$

or writing this in the form

$$
\{012\}-\{312\}+\{023\}-\{013\}=0,
$$

suppose 2 is here the consecutive point $1+\delta 1$; then

$$
\{012\}-\{312\},=\frac{1^{22} .023-12^{2} \cdot 013}{012.312}
$$

becomes $=\{0 t 13\} \delta \omega_{1}$ : we have also

and the result is

$$
\{023\}=\{013\}+\partial_{1}\{c 13\} \delta \omega_{1}
$$

$$
-\{0 t 13\}+\partial_{1} 013=0,
$$

that is, $\partial_{1}\{013\}=\{0 t 13\}$. The form in the case of the cubic $x^{3}+y^{3}+z^{3}+6 l x y z=0$, is $=$

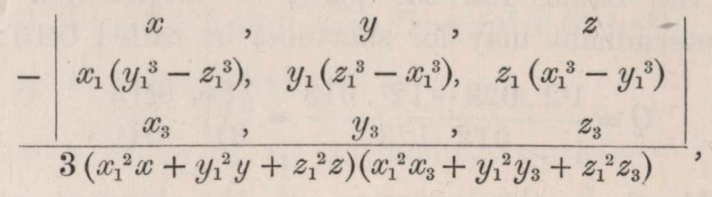

i.e. the differential coefficient of $\{013\}$ in regard to the parametric point 1 is $=\{0 t 13\}$, the symbol for the case where the parametric line is the tangent at 1 . 
Fixed Curve a Cubic: the Parametric Points corresponding points. Art. Nos. 107 to 110.

107. The parametric points 1,2 are taken to be corresponding points, that is, such that the tangents at these points meet at a point, say 3 , on the cubic. We may from 3 draw two other tangents, touching the cubic, say at the points $1^{\prime}$ and $2^{\prime}$. The four points $1,2,1^{\prime}, 2^{\prime}$ are then such that the lines $12,1^{\prime} 2^{\prime}$ meet in a point, say 4, of the cubic; and moreover 3, 4 are corresponding points.

We may take $(x, y, z),=(1,0,0),(0,1,0),(0,0,1)$ for the coordinates of the points 1, 2, 3 respectively: $x=0, y=0$ are thus the equations of the lines 32,31 respectively, and $z=0$ is the equation of the line 12 , viz. we have $z=012$. Taking $x-M_{1} y=0, x-M_{2} y=0$, for the equations of the tangents $31^{\prime}, 32^{\prime}$ respectively, and $\zeta=0$ for the equation of the line $1^{\prime} 2^{\prime}$ joining their points of contact, where $\zeta$ is a properly determined linear function of $(x, y, z)$, it is to be shown that the differential $Q d \omega$ may be taken to be $\frac{\zeta d \omega}{z}$, and that this is $=\frac{1}{2}\left(\frac{d x}{x}-\frac{d y}{y}\right):$ the affected theorem thus assumes a special form, which will be noticed.

108. The cubic passes through the points $(x=0, z=0)$ and $(y=0, z=0)$, the tangents at these points being $x=0$, and $y=0$ respectively: also through the point $x=0, y=0$ : its equation thus is

and writing

$$
f,=g z^{2} x+2 l z x y+i z^{2} y+h x^{2} y+k x y^{2},=0,
$$

$$
d \omega=\frac{x d y-y d x}{\frac{d f}{d z}}
$$

we have

$$
\frac{d f}{d z}=2(g z x+l x y+i z y)
$$

which, from the equation of the curve written in the form

or say

$$
z(g z x+l x y+i z y)+x y(h x+k y+l z)=0,
$$

becomes

$$
z(g z x+l x y+i z y)+x y \zeta=0
$$

and we thus have

$$
=\frac{-2 x y \zeta}{z}
$$

$$
d \omega=\frac{-z}{2 x y \zeta}(x d y-y d x),=\frac{1}{2} \frac{z}{\zeta}\left(\frac{d x}{x}-\frac{d y}{y}\right)
$$

where $\zeta=h x+k y+l z$. To find the meaning of $\zeta$, observe that the line $x-M y=0$ meets the curve in the point $(x=0, y=0)$, and in two other points determined by the equation

$$
z^{2}(g m+i)+2 z y l M+y^{2}\left(h M^{2}+k M\right)=0 ;
$$

this line will be a tangent if

$$
(g M+i)(h M+k)-l^{2} M=0,
$$


and we then have at the point of contact $(h M+k) y+l z=0$; and writing this in the form $h x+k y+l z=0$, we see that the equation $\zeta=0$ is satisfied at the point of contact of each of the two tangents $x-M_{1} y=0, x-M_{2} y=0$; viz. $\zeta=0$ is the equation of the line joining the two points of contact. Moreover, from the equation of the curve written in the foregoing form

$$
z(g z x+l x y+i z y)+x y \zeta=0
$$

it appears that the lines $z=0, \zeta=0$, meet on the curve; or, what is the same thing, that the line $\zeta=0$ passes through the residue of the parametric points 1,2 .

109. The function $\zeta$ at 1 becomes $=h$, and this is the value of $3.1^{22}$; in fact,

$$
\begin{aligned}
3.1^{22} & =\left(x_{2} \frac{d}{d x_{1}}+y_{2} \frac{d}{d y_{1}}+z_{2} \frac{d}{d z_{1}}\right) f_{1}, \quad\left(x_{2}, y_{2}, z_{2}\right)=(0,1,0) \\
& =\frac{d f_{1}}{d y_{1}} \\
& =2 l z_{1} x_{1}+i z_{1}^{2}+h x_{1}^{2}+2 k x_{1} y_{1}, \quad\left(x_{1}, y_{1}, z_{1}\right)=(1,0,0) \\
& =h .
\end{aligned}
$$

We have thus $\zeta$, satisfying the required conditions for the major function: and the differential $Q d \omega$ may therefore be taken to be $=\frac{\zeta}{z} d \omega$, that is, we have

$$
Q d \omega=\frac{1}{2}\left(\frac{d x}{x}-\frac{d y}{y}\right)
$$

The affected theorem thus becomes

$$
\Sigma_{\frac{1}{2}}\left(\frac{d x}{x}-\frac{d y}{y}\right)=-\frac{\delta \phi_{1}}{\phi_{1}}+\frac{\delta \phi_{2}}{\phi_{2}}
$$

110. The meaning of this will be better understood from the integral form. Integrating each side, and assuming that the superior limits are given by a line $\phi$ which cuts the cubic in the points $4,5,6$, and the inferior limits by a line $\psi$ which cuts the cubic in the points 7,8 , 9 , we find

that is,

$$
\log \frac{x_{4} x_{5} x_{6}}{x_{7} x_{8} x_{9}}-\log \frac{y_{4} y_{5} y_{6}}{y_{7} y_{8} y_{9}}=2 \log \frac{\phi_{2}}{\psi_{2}} \frac{\psi_{1}}{\phi_{1}}
$$

$$
\frac{x_{4} x_{5} x_{6} y_{7} y_{8} y_{9}}{x_{7} x_{8} x_{9} y_{4} y_{5} y_{6}}=\left(\begin{array}{l}
\phi_{2} \psi_{1} \\
\psi_{2} \phi_{1}
\end{array}\right)^{2}
$$

where $\phi_{1}, \psi_{1}, \phi_{2}, \psi_{2}$ denote the values of the linear functions $\phi, \psi$ at the points 1 and 2 respectively. We have a cubic cut by the lines $\phi, \psi, x, y$ in the points $4,5,6 ; 7,8,9 ; 2,2^{\prime}, 3$ and $1,1^{\prime}, 3$ respectively: where for the moment $1^{\prime}, 2^{\prime}$ are written to denote the points on the curve consecutive to 1 and 2 respectively. Hence, by a known theorem in transversals,

$$
\left(\frac{x}{y}\right)_{456} \div\left(\frac{x}{y}\right)_{789}=\left(\frac{\phi}{\psi}\right)_{22^{\prime} 3} \div\left(\frac{\phi}{\psi}\right)_{11^{\prime} 3}
$$


that is,

$$
\frac{x_{4} x_{5} x_{6} y_{7} y_{8} y_{9}}{x_{7} x_{8} x_{9} y_{4} y_{5} y_{6}}=\frac{\psi_{1} \psi_{1^{\prime}} \psi_{3} \cdot \phi_{2} \phi_{2^{\prime}} \phi_{3}}{\psi_{2} \psi_{2^{\prime}} \psi_{3} \cdot \phi_{1} \phi_{1^{\prime}} \phi_{3}}
$$

which, dividing out the $\phi_{3} \psi_{3}$, and writing 1,2 in place of $1^{\prime}, 2^{\prime}$, becomes

$$
=\left(\frac{\phi_{2} \psi_{1}}{\phi_{1} \psi_{2}}\right)^{2}
$$

agreeing with the result just obtained.

Aronhold's Cubic Transformation. Art. Nos. 111 to 119.

111. This was obtained in the paper "Algebraische Reduction des Integrals $\int F^{\prime}(x, y) d x$, u. s. w." Berl. Monatsb., April, 1861, pp. 462-468. I give in the first place the analytical results, independently of the general theory, with the values for the canonical form $f,=\frac{1}{3}\left(x^{3}+y^{3}+z^{3}+6 l x y z\right),=0$, of the cubic.

$$
\begin{array}{ll}
T \text { sextic invariant, } & =1-20 l^{3}-8 l^{6}, \\
S \quad \text { quartic " (Aronhold's }) & =-4\left(l-l^{4}\right), \\
R \quad \text { discriminant } & =\left(1+8 l^{3}\right)^{3}, \\
P=3 h \alpha^{2} 0 & =\left\{-3 l^{2} \alpha^{2}+\left(1+2 l^{3}\right) \beta \gamma\right\} x+\& \mathrm{c} . \\
Q=f \mathrm{a}^{2} 0 & =\left(\alpha^{2}+2 l \beta \gamma\right) x+\& \mathrm{c} ., \\
B=f \mathrm{a}^{2} 0 & =\left(\mathrm{a}^{2}+2 l \mathrm{bc}\right) x+\& \mathrm{c} ., \\
C=f \mathrm{a} 0^{2} & =\mathrm{a}\left(x^{2}+2 l y z\right)+\& \mathrm{c} ., \\
D=f 0^{3} & =x^{3}+y^{3}+z^{3}+6 l x y z,
\end{array}
$$

where a, b, $c=\alpha\left(\beta^{3}-\gamma^{3}\right), \beta\left(\gamma^{3}-\alpha^{3}\right), \gamma\left(\alpha^{3}-\beta^{3}\right)$.

Then we have

$$
2 T Q^{4}+6 S P Q^{3}+8 P^{3} Q=-R^{\frac{2}{3}}\left(6 C^{2}-8 B D\right),
$$

viz. this equation, where each side is a quartic function $(x, y, z)^{4}$, is an identity when $(\alpha, \beta, \gamma)$ are connected by the equation, $f \alpha^{3},=\alpha^{3}+\beta^{3}+\gamma^{3}+6 l \alpha \beta \gamma,=0$; and further,

$$
Q d P-P d Q=-R^{\frac{1}{3}}\{\mathrm{a}(y d z-z d y)+\mathrm{b}(z d x-x d z)+\mathrm{c}(x d y-y d x)\} .
$$

Hence writing

$$
\lambda=\frac{6 h \alpha^{2} 0}{f \alpha^{2} 0},=\frac{2 P}{Q},=\frac{2\left(\left\{-3 l^{2} \alpha^{2}+\left(1+2 l^{3}\right) \beta \gamma\right\} x+\& c .\right)}{\left(\alpha^{2}+2 l \beta \gamma\right) x+\& c .},
$$

we have

$$
Q^{4}\left(\lambda^{3}-3 S \lambda-2 T\right)=R^{2}\left(6 C^{2}-8 B D\right),
$$

and

$$
Q^{2} d \lambda,=2(Q d P-P d Q)=-2 R^{\frac{1}{3}}\{a(y d z-z d y)+\& c .\}
$$


112. Supposing now that $(x, y, z)$ are the coordinates of a point on the cubic, then $D=0$; and taking the square root of each side of the first equation, we may write

We have

$$
\begin{aligned}
& Q^{2} \sqrt{\lambda^{3}-3 S \lambda-2 T}=-R^{\frac{1}{3}} \sqrt{6} \cdot C, \\
& Q^{2} d \lambda \quad=-2 R^{\frac{1}{3}}\{a(y d z-z d y)+\& c .\} .
\end{aligned}
$$

whence

$$
d \omega=\frac{y d z-z d y}{x^{2}+2 l y z}=\frac{z d x-x d z}{y^{2}+2 l z x}=\frac{x d y-y d x}{z^{2}+2 l x y}
$$

$$
d \omega=\frac{\mathrm{a}(y d z-z d y)+\& c .}{C}
$$

and we consequently have

or, as this may also be written,

$$
\frac{d \lambda}{\sqrt{\lambda^{3}-3 S \lambda-2 T}}=\frac{2}{\sqrt{6}}
$$

$$
\frac{d \lambda}{\sqrt{4 \lambda^{3}-12 S \lambda-8 T}}=\frac{1}{\sqrt{6}} d \omega
$$

which, if $12 S, 8 T$ are put $=g_{2}, g_{3}$ respectively, takes the Weierstrassian form

$$
\frac{d \lambda}{\sqrt{4 \lambda^{3}-g_{2} \lambda-g_{3}}}=\frac{1}{\sqrt{6}} d \omega
$$

The conclusion is that for the cubic curve, taking $\lambda$ a quotient of two linear functions of $(x, y, z)$, the differential $d \omega$ is transformed into $d \lambda \div$ square root of a cubic function of $\lambda$ : viz. we have thus a form of differential, not the same, but such as that which belongs to the ordinary theory of elliptic functions, and which has been adopted by Weierstrass as a canonical form.

113. The transformation depends on the arbitrary point $(\alpha, \beta, \gamma)$ of the cubic: the point $(a, b, c)$ is the tangential of this point, viz. the point of intersection of the tangent at $(\alpha, \beta, \gamma)$ with the cubic: we can from $(a, b, c)$ draw four tangents to the cubic, viz. the tangent at $(\alpha, \beta, \gamma)$ and three other tangents: the equations of the four tangents being $\frac{2 P}{Q},=\frac{6 h \alpha^{2} 0}{f \alpha^{2} 0},=\infty, \lambda_{1}, \lambda_{2}, \lambda_{3}$ respectively; where $\lambda_{1}, \lambda_{2}, \lambda_{3}$ are the roots of the equation $\lambda^{3}-3 S \lambda-2 T=0$.

Suppose for a moment that $(\alpha, \beta, \gamma)$ is a point not on the cubic curve, and write $A=\alpha^{3}+\beta^{3}+\gamma^{3}+6 l \alpha \beta \gamma$. We have

$$
A^{2} D^{2}+4 A C^{3}+4 B^{3} D-3 B^{2} C^{2}-6 A B C D=0,
$$

for the equation of the six tangents which can be drawn from the point $(\alpha, \beta, \gamma)$ to the cubic: when $(\alpha, \beta, \gamma)$ is on the cubic, $A=0$, and the equation becomes $B^{2}\left(4 B D-3 C^{2}\right)=0$, where $B=0$ is the equation of the tangent at the point $(\alpha, \beta, \gamma)$ : throwing out the factor $B^{2}$, we have $4 B D-3 C^{2}=0$ for the equation of the four tangents from $(\alpha, \beta, \gamma)$ to the curve; viz. the equation of the four tangents is

$$
2 T Q^{4}+6 S P Q^{3}+8 P^{3} Q=0
$$


or, as this may be written,

$$
Q\left(2 P-\lambda_{1} Q\right)\left(2 P-\lambda_{2} Q\right)\left(2 P-\lambda_{3} Q\right)=0,
$$

viz. the equations of the four tangents are as is mentioned above; it was, in fact, by these geometrical considerations that Aronhold obtained his results.

114. The foregoing expression for $Q d P-P d Q$, say

$$
Q d P-P d Q=\left(1+8 l^{3}\right)\{\mathrm{a}(y d z-z d y)+\mathrm{b}(z d x-x d z)+\mathrm{c}(x d y-y d x)\},
$$

may be verified without difficulty. Writing for a moment

we have

$$
\begin{aligned}
Q d P-P d Q= & (A x+B y+C z)(L d x+M d y+N d z) \\
& -(A d x+B d y+C d z)(L x+M y+N z) \\
= & (B N-C M)(y d z-z d y)-\& c . ;
\end{aligned}
$$

which proves the theorem.

$$
\begin{aligned}
B N-C M= & \left\{-3 l^{2} \beta^{2}+\left(1+2 l^{3}\right) \gamma \alpha\right\}\left(\gamma^{2}+2 l \alpha \beta\right) \\
& -\left\{-3 l^{2} \gamma^{2}+\left(1+2 l^{3}\right) \alpha \beta\right\}\left(\beta^{2}+2 l \gamma \alpha\right) \\
= & -6 l^{3} \alpha \beta^{3}+\left(1+2 l^{3}\right) \alpha \gamma^{3}+6 l^{3} \alpha \gamma^{3}-\left(1+2 l^{3}\right) \alpha \beta^{3}, \\
= & -\left(1+8 l^{3}\right) \alpha\left(\beta^{3}-\gamma^{3}\right) \\
= & -\left(1+8 l^{3}\right) a
\end{aligned}
$$

115. I content myself with a partial verification of the identity

$$
2 T Q^{4}+6 S P Q^{3}-8 P^{3} Q=-\left(1+8 l^{3}\right)^{2}\left(6 C^{2}-8 B D\right) .
$$

Writing herein $x, y, z=1,-1,0$, we have $D=0$, and the equation becomes

where now

$$
2 T Q^{4}+6 S P Q^{3}-8 P^{3} Q+6\left(1+8 l^{3}\right)^{2} C^{2}=0,
$$

$$
\begin{aligned}
& Q=(\alpha-\beta)(\alpha+\beta-2 l \gamma), P=(\alpha-\beta)\left\{-3 l^{2}(\alpha+\beta)-\left(1+2 l^{3}\right) \gamma\right\}, \\
& C=\mathrm{a}+\mathrm{b}-2 l \mathrm{c}, \quad=(\alpha-\beta)\left\{-\alpha \beta^{2}-\alpha^{2} \beta-\gamma^{3}-2 l \gamma\left(\alpha^{2}+\alpha \beta+\beta^{2}\right)\right\},
\end{aligned}
$$

which, putting therein $-\gamma^{3}=\alpha^{3}+\beta^{3}+6 l \alpha \beta \gamma$, becomes

Hence writing

$$
=(\alpha-\beta)^{2}\left(\alpha+\beta-2 l_{\gamma}\right) .
$$

we have

$$
X=\alpha+\beta-2 l \gamma, \quad Y=-3 l^{2}(\alpha+\beta)-\left(1+2 l^{3}\right) \gamma,
$$

$$
Q, P, C=(\alpha-\beta) X, \quad(\alpha-\beta) Y, \quad(\alpha-\beta)^{3} X:
$$

substituting these values, the factor $(\alpha-\beta)^{4} X$ divides out, and the equation becomes

$$
2 T X^{3}+6 S X^{2} Y-8 Y^{3}+6\left(1+8 l^{3}\right)^{2}(\alpha-\beta)^{2} X=0 .
$$

To complete the verification, observe that we have $Y+3 l^{2} X=-\left(1+8 l^{3}\right) \gamma$, whence

$$
-Y^{3}=\left(1+8 l^{3}\right)^{3} \gamma^{3}+9\left(1+8 l^{3}\right)^{2} l^{2} \gamma^{2} X+27\left(1+8 l^{3}\right) l^{4} \gamma X^{2}+27 l^{6} X^{3},
$$


and herein $-\gamma^{3}=\alpha^{3}+\beta^{3}+6 l \alpha \beta \gamma$, whence

that is,

$$
-\gamma^{3}+\alpha \beta X=(\alpha+\beta)^{3}=(X+2 l \gamma)^{3}=X^{3}+6 l \gamma X^{2}+12 l^{2} \gamma^{2} X+8 l^{3} \gamma^{3},
$$

$$
-\left(1+8 l^{3}\right) \gamma^{3}=X^{3}+6 l \gamma X^{2}+\left(12 l^{2} \gamma^{2}-3 \alpha \beta\right) X .
$$

Hence the equation to be verified becomes

$$
\begin{aligned}
2 T X^{3}+6 S X^{2} Y-8 & \left\{\begin{array}{l}
\left(1+8 l^{3}\right)^{2}\left[X^{3}+6 l \gamma X^{2}+\left(12 l^{2} \gamma^{2}-3 \alpha \beta\right) X\right] \\
-\left(1+8 l^{3}\right)^{2} 9 l^{2} \gamma^{2} X \\
-\left(1+8 l^{3}\right) 27 l^{4} \gamma X^{2} \\
-27 l^{6} X^{3} \\
+6\left(1+8 l^{3}\right)^{2}(\alpha-\beta)^{2} X=0
\end{array}\right\}
\end{aligned}
$$

viz. throwing out the factor $X$, this is

$$
\left\{2 T-8\left(1+8 l^{3}\right)^{2}+216 l^{6}\right\} X^{2}+6 S X Y-48\left(1+8 l^{3}\right)^{2} l \gamma X+216\left(1+8 l^{3}\right) l^{4} \gamma X
$$

$$
-\left(1+8 l^{3}\right)^{2}\left\{96 l^{2} \gamma^{2}-24 \alpha \beta-72 l^{2} \gamma^{2}-6(\alpha-\beta)^{2}\right\}=0,
$$

where the last term is

viz. this is

$$
=+6\left(1+8 l^{3}\right)^{2}\left\{(\alpha+\beta)^{2}-4 l^{2} \gamma^{2}\right\},
$$

$$
=6\left(1+8 l^{3}\right)^{2}(\alpha+\beta+2 l \gamma) X,
$$

and there is again the factor $X$ which can be thrown out: the equation thus becomes

$$
\begin{aligned}
{\left[2 T-8\left(1+8 l^{3}\right)^{2}+216 l^{6}\right] X+6 S Y-48\left(1+8 l^{3}\right)^{2} l \gamma+} & 216\left(1+8 l^{3}\right) l^{4} \gamma \\
& +6\left(1+8 l^{3}\right)^{2}(\alpha+\beta+2 l \gamma)=0 .
\end{aligned}
$$

This may be written

$$
\begin{aligned}
{\left[2 T-8\left(1+8 l^{3}\right)^{2}+216 l^{6}\right] X+6 S\left[-3 l^{2} X-\left(1+8 l^{3}\right) \gamma\right]-48\left(1+8 l^{3}\right)^{2} l \gamma } \\
+216\left(1+8 l^{3}\right) l^{4} \gamma+6\left(1+8 l^{3}\right)^{2}(X+4 l \gamma)=0,
\end{aligned}
$$

or, finally, it is

$$
\begin{aligned}
& {\left[2 T-8\left(1+8 l^{3}\right)^{2}+216 l^{6}-18 l^{2} S+6\left(1+8 l^{3}\right)^{2}\right] X} \\
& +\left[-6 l^{-1} S-48\left(1+8 l^{3}\right)+216 l^{3}+24\left(1+8 l^{3}\right)\right]\left(1+8 l^{3}\right) l \gamma=0 ;
\end{aligned}
$$

substituting for $T, S$ their values $1-20 l^{3}-8 l^{6}$ and $-4 l+4 l^{4}$ respectively, the coefficients of $X$ and $\left(1+8 l^{3}\right) l_{\gamma}$ are separately $=0$, and the equation is thus verified.

116. The foregoing equation $\lambda=\frac{6 h \alpha^{2} 0}{f \alpha^{2} 0}$, regarding therein $\bar{\lambda}$ as an arbitrary parameter and $(x, y, z)$ as current coordinates, is the equation of an arbitrary line through the point $(a, b, c)$ of the cubic: it meets the cubic in two other points depending, of course, on the value of $\lambda$; and the coordinates of either of these is thus expressible, irrationally, in terms of $\lambda$, the expressions involving the radical $\sqrt{\lambda^{3}-3 S \lambda-2 T}$ : from the values of $x, y, z$ in terms of $\lambda$, we should be able to deduce the foregoing equation 
$\frac{2}{\sqrt{6}} d \omega=\frac{d \lambda}{\sqrt{\lambda^{3}-3 S \lambda-2 T}}$. The expressions assume a peculiarly simple form when $(\alpha, \beta, \gamma)$, instead of being an arbitrary point of the cubic, is a point of inflexion of the cubic ; and it is easy to see $a$ priori why this is so: in fact, if we assume

$$
x: y: z=u+\alpha \sqrt{\Lambda}: v+\beta \sqrt{\Lambda}: w+\gamma \sqrt{\Lambda},
$$

where $u, v, w$ are linear functions and $\Lambda$ a cubic function of $\lambda$; then the locus is a cubic curve, and corresponding to the value $\lambda=\infty$, we have $x: y: z=\alpha: \beta: \gamma$, viz. the curve passes through the point $(\alpha, \beta, \gamma)$ : moreover, it can be shown that this point is an inflexion of the curve; expressions of the foregoing simple form thus only exist in the case where the point $(\alpha, \beta, \gamma)$ is an inflexion; and the formulæ referring to an arbitrary point $(\alpha, \beta, \gamma)$ of the curve are necessarily of a more complex form.

117. To work this out, we start from the foregoing equation

which, putting therein

$$
\lambda=\frac{6 h \alpha^{2} 0}{f \alpha^{2} 0}=\frac{2\left(\left\{-3 l^{2} \alpha^{2}+\left(1+2 l^{3}\right) \beta \gamma\right\} x+\& c .\right)}{\left(\alpha^{2}+2 l \beta \gamma\right) x+\& c .}
$$

and

$$
L=\lambda+6 l^{2}, \quad M=l \lambda-\left(1+2 l^{3}\right),
$$

$$
A, B, C=L \alpha^{2}+2 M \beta \gamma, \quad L \beta^{2}+2 M \gamma \alpha, \quad L \gamma^{2}+2 M \alpha \beta,
$$

becomes $A x+B y+C z=0$, the equation of a line through the point

$$
\mathrm{a}, \mathrm{b}, \mathrm{c},=\alpha\left(\beta^{3}-\gamma^{3}\right), \quad \beta\left(\gamma^{3}-\alpha^{3}\right), \gamma\left(\alpha^{3}-\beta^{3}\right),
$$

as before: and we have to find the intersections of this line with the cubic

We have

$$
x^{3}+y^{3}+z^{3}+6 l x y z=0 .
$$

$$
C^{3}\left(x^{3}+y^{3}\right)-(A x+B y)^{3}-6 l C^{2}(A x+B y) x y=0:
$$

the cubic function contains as we know the factor $b x-a y$, and in the remaining quadric factor it is easy to calculate the coefficients of $x^{2}$ and $y^{2}$ : we thus obtain the identity

$$
\begin{gathered}
C^{3}\left(x^{3}+y^{3}\right)-(A x+B y)^{3}-6 l C^{2}(A x+B y) x y \\
=(\mathrm{b} x-\mathrm{a} y)\left\{\left[\left(-\beta^{2}-6 l \gamma \alpha\right) L^{3}+6 \alpha \gamma L^{2} M-8 \beta^{2} M^{3}\right] x^{2}\right. \\
+2 H \quad x y \\
\left.+\left[\left(-\alpha^{2}-6 l \alpha \beta\right) L^{3}+6 \beta \gamma L^{2} M-8 \alpha^{2} M^{3}\right] y^{2}\right\},
\end{gathered}
$$

from which the as yet unknown coefficient $2 H$ is to be obtained. This is most easily effected by assuming $x, y=\alpha,-\beta$; values which give

$$
x^{3}+y^{3}=\alpha^{3}-\beta^{3}, \quad A x+B y=L\left(\alpha^{3}-\beta^{3}\right), \quad \mathrm{b} x-\mathrm{a} y=-\alpha \beta\left(\alpha^{3}-\beta^{3}\right):
$$

the whole equation becomes divisible by $\alpha^{3}-\beta^{3}$ and, omitting this factor, we have

$$
\begin{aligned}
& C^{3}-L^{3}\left(\alpha^{3}-\beta^{3}\right)^{2}+6 l C^{2} L \alpha \beta \\
& \qquad=\alpha \beta\left\{\left[2 \alpha^{2} \beta^{2}+6 l \gamma\left(\alpha^{3}+\beta^{3}\right)\right] L^{3}-6 \gamma\left(\alpha^{3}+\beta^{3}\right) L^{2} M+16 \alpha^{2} \beta^{2} M^{3}\right\}+2 H \alpha^{2} \beta^{2}, \\
& \text { C. XII. }
\end{aligned}
$$


where for $C$ is to be substituted its value $L \gamma^{2}+2 M a \beta$. We may also reduce by $\alpha^{3}+\beta^{3}+\gamma^{3}+6 l \alpha \beta \gamma=0$. The left-hand side is

$$
C^{3}-L^{3}\left(\alpha^{3}+\beta^{3}\right)^{2}-4 \alpha^{3} \beta^{3} L^{3}+6 l C^{2} L \alpha \beta,
$$

which after reduction is found to contain the factor $\alpha \beta$; and omitting this factor and reducing also the right-hand side, the whole equation becomes

$$
\begin{aligned}
& L^{3}\left(-6 l \gamma^{4}-36 l^{2} \gamma^{2} \alpha \beta+4 \alpha^{2} \beta^{2}\right) \quad=L^{3}\left(-6 l \gamma^{4}-36 l^{2} \gamma^{2} \alpha \beta+2 \alpha^{2} \beta^{2}\right) \\
& +L^{2} M\left(6 \gamma^{4}+24 l \gamma^{2} \alpha \beta\right) \quad+L^{2} M\left(6 \gamma^{4}+36 l \gamma^{2} \alpha \beta\right) \\
& +L M^{2}\left(12 \gamma^{2} \alpha \beta+24 l \alpha^{2} \beta^{2}\right) \\
& +M^{3}\left(8 \alpha^{2} \beta^{2}\right) \quad+M^{3}\left(16 \alpha^{2} \beta^{2}\right) \\
& +2 H \alpha \beta
\end{aligned}
$$

omitting here the terms which destroy each other, the equation again divides by $\alpha \beta$ and we thus obtain the value of $H$; and the required identity is

$$
\begin{aligned}
& C^{3}\left(x^{3}+y^{3}\right)-(A x+B y)^{3}-6 l C^{2}(A x+B y) x y \\
&=(\mathrm{b} x-\mathrm{a} y)\left\{\left[\left(-\beta^{2}-6 l \gamma \alpha\right) L^{3}+6 \alpha \gamma L^{2} M-8 \beta^{2} M^{3}\right] x^{2}\right. \\
&+ {\left[\alpha \beta L^{3}-6 l \gamma^{2} L^{2} M+\left(6 \gamma^{2}+12 l \alpha \beta\right) L M^{2}-4 \alpha \beta M^{3}\right] 2 x y } \\
&+ {\left.\left[\left(-\alpha^{2}-6 l \beta \gamma\right) L^{3}+6 \beta \gamma L^{2} M-8 \alpha^{2} M^{3}\right] y^{2}\right\} . }
\end{aligned}
$$

Hence putting for shortness

$$
\begin{array}{lr}
\mathfrak{A}=\left(\alpha^{2}+6 l \beta \gamma\right) L^{3}-6 \beta \gamma L^{2} M & +8 \alpha^{2} M^{3}, \\
\mathfrak{B}=\left(\beta^{2}+6 l \gamma \alpha\right) L^{3}-6 \alpha \gamma L^{2} M & +8 \beta^{2} M^{3}, \\
\mathfrak{S}= & \alpha \beta L^{3}-6 l \gamma^{2} L^{2} M+\left(6 \gamma^{2}+12 l \alpha \beta\right) L M^{2}-4 \alpha \beta M^{3},
\end{array}
$$

the equation in $(x, y)$ is

giving

$$
\mathfrak{B} x^{2}-2 \mathfrak{g} x y+\mathfrak{2} y^{2}=0,
$$

or say

$$
\mathfrak{B} x-\mathfrak{S} y=\sqrt{\mathfrak{H}^{2}}-\mathfrak{A} \mathfrak{B} y,
$$

$$
x: y=\mathfrak{H}+\sqrt{\mathfrak{S}^{2}-} \overline{\mathfrak{I} \mathfrak{B}}: \mathfrak{B} .
$$

We find without difficulty, reducing always by $\alpha^{3}+\beta^{3}+\gamma^{3}+6 l \alpha \beta \gamma=0$,

$$
\begin{aligned}
\frac{1}{6}\left(\mathfrak{H}^{2}-\mathfrak{2 B}\right)= & L^{6} \\
& +\left(-\gamma^{4}+4 l \alpha \beta \gamma^{2}\right) L^{5} M \\
& +\left(6 l^{2} \gamma^{4}-4 \alpha \beta \gamma^{2}+4 l \alpha^{2} \beta^{2}\right) L^{4} M^{2} \\
& +\left(-4 l \gamma^{4}+24 l^{2} \alpha \beta \gamma^{2}-4 \alpha^{2} \beta^{2}\right) L^{3} M^{3} \\
& +\left(-2 \gamma^{4}-16 l \alpha \beta \gamma^{2}+24 l \alpha^{2} \beta^{2}\right) L^{2} M^{4} \\
& +\left(-8 \alpha \beta \gamma^{2}-16 l \alpha^{2} \beta^{2}\right) L M^{5} \\
& +\left(-8 \alpha^{2} \beta^{2}\right) M^{6},
\end{aligned}
$$


which is

But we have

$$
=(l L-M)\left(L^{3}+6 l L M^{2}+2 M^{3}\right)\left(\gamma^{2} L+2 \alpha \beta M\right)^{2} .
$$

$$
\begin{aligned}
l L-M & =1+8 l^{3}, \\
L^{3}+6 l L M^{2}+2 M^{3} & =\left(1+8 l^{3}\right)\left(\lambda^{3}-3 S \lambda-2 T\right),
\end{aligned}
$$

and the equation thus is

$$
\frac{1}{6}\left(\mathfrak{H}^{2}-\mathfrak{2 B}\right)=\left(1+8 l^{3}\right)^{2}\left(\lambda^{3}-3 S \lambda-2 T\right)\left[\left(\gamma^{2}+2 l \alpha \beta\right) \lambda+\left\{6 l^{2} \gamma^{2}-2\left(1+2 l^{3}\right) \alpha \beta\right\}\right]^{2},
$$

showing that the solution involves the radical $\sqrt{\lambda^{3}-3 S \lambda-2 T}$.

118. If $(\alpha, \beta, \gamma)$ is the inflexion $(1,-1,0)$, the expression for $\lambda$ is here

the equation in $(x, y)$ is

$$
\lambda=\frac{-6 l^{2} x-6 l^{2} y-2\left(1+2 l^{3}\right) z}{x+y-2 l z}
$$

$$
\left(L^{3}+8 M^{3}\right) x^{2}+\left(2 L^{3}+24 l L M^{2}-8 M^{3}\right) x y+\left(L^{3}+8 M^{3}\right) y^{2}=0,
$$

or, as this may be written,

say

$$
\left(L^{3}+6 l L M^{2}+2 M^{3}\right)(x+y)^{2}+\left(-6 l L M^{2}+6 M^{3}\right)(x-y)^{2}=0,
$$

$$
\left(L^{3}+6 l L M^{2}+2 M^{3}\right)(x+y)^{2}=6 M^{2}(l L-M)(x-y)^{2} ;
$$

viz. we thus have

$$
\sqrt{\lambda^{3}-3 S \lambda-2 T}(x+y)=M \sqrt{6}(x-y),
$$

or, substituting for $M$ its value,

whence also

$$
\begin{aligned}
& x=\sqrt{6}\left\{l \lambda-\left(1+2 l^{3}\right)\right\}+\sqrt{\lambda^{3}-3 S \lambda-2 T,} \\
& y=\sqrt{6}\left\{l \lambda-\left(1+2 l^{3}\right)\right\}-\sqrt{\lambda^{3}-3 S \lambda+2 T},
\end{aligned}
$$

$$
z=\sqrt{6}\left(\lambda+6 l^{2}\right)
$$

these values satisfying identically

and

$$
\left(\lambda+6 l^{2}\right)(x+y)-2\left[l \lambda-\left(1+2 l^{3}\right)\right] z=0,
$$

$$
x^{3}+y^{3}+z^{3}+6 l x y z=0 \text {. }
$$

119. Starting from these values we, in fact, easily obtain

$$
\begin{aligned}
x d y-y d x & =\frac{-\sqrt{6} d \lambda}{\sqrt{\lambda^{3}-3 S \lambda-2 T}} \times \\
\left\{l \lambda^{3}+\left(-3-6 l^{3}\right) \lambda^{2}+\left(-12 l^{2}+12 l^{5}\right) \lambda+\left(-8 l-92 l^{4}-8 l^{7}\right)\right\} & \\
z^{2}+2 l x y & =-2\{\text { Do. }\}
\end{aligned}
$$

and hence

$$
d \omega=\frac{x d y-y d x}{z^{2}+2 l x y}=\frac{\frac{1}{2} \sqrt{6} d \lambda}{\sqrt{\lambda^{3}-3 S \lambda-2 T}}
$$

The same result might of course have been obtained from the values of $x, z$ or $y, z$, the factor which divides out being in each of these cases irrational. 
The Cubic $y^{2}=x(1-x)\left(1-k^{2} x\right)$. Art. Nos. 120 to 130 (several sub-headings).

120. The curve is a semi-cubical parabola, symmetrical in regard to the axis of $x$; and if, as usual, $k^{2}$ is taken to be real, positive and less than 1 , then the curve consists of an oval, and an infinite portion which may be called the anguis. (See Figure.)

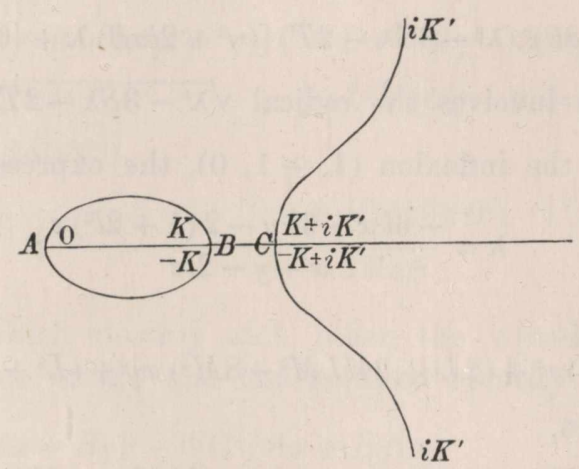

The equation is satisfied by

$$
\begin{aligned}
& x=\operatorname{sn}^{2} u, \\
& y=\operatorname{sn} u \text { cn } u \operatorname{dn} u .
\end{aligned}
$$

Observe that the periods for these combinations of the elliptic functions are $2 K, 2 i K^{\prime}$; in fact,

$$
\begin{aligned}
& \text { sn }(u+2 K)=-\operatorname{sn} u, \quad \text { sn }\left(u+2 i K^{\prime}\right)=\quad \text { sn } u, \\
& \text { cn }, \quad=-\mathrm{cn} u \text {, cn , } \quad=-\mathrm{cn} u \text {, } \\
& \operatorname{dn} \quad=\quad=\operatorname{dn} u, \operatorname{dn}, \quad=-\operatorname{dn} u,
\end{aligned}
$$

whence the $\mathrm{sn}^{2}$ and the $\mathrm{sn} . \mathrm{cn} . \mathrm{dn}$ are each unaltered by the change of $u$ into $u+2 K$ or $u+2 i K^{\prime}$. Hence to a given point $(x, y)$ on the curve, the argument $u$ is not $=$ a determinate value $u_{0}$, for it may be equally well taken to be $=u_{0}+2 m K+2 m^{\prime} i K^{\prime}$, where $m, m^{\prime}$ are any positive or negative integers whatever: we express this by $u \equiv u_{0}$, or say $u$. is congruent to $u_{0}$. But when $u$ is thus given by a congruence $u \equiv u_{0}$ where $u_{0}$ has a determinate value, the point on the curve is uniquely determined. It is, however, to be noticed that a congruence $2 u \equiv u_{0}$ does not uniquely determine the point on the curve: there are, in fact, four incongruent values of $u$, viz.

$$
\frac{1}{2} u_{0}, \frac{1}{2} u_{0}+K, \frac{1}{2} u_{0}+i K^{\prime}, \frac{1}{2} u_{0}+K+i K^{\prime},
$$

and the point on the curve is thus one of the four points belonging to these values of $u$ respectively.

121. If, to fix the ideas, we select for each point of the curve one of the congruent values of the argument, we may assume for the oval, $u$ real: at $A, u=0$; from $A$ to $B$ above the axis $u$ positive and at $B,=K$; below the axis $u$ negative and at $B,=-K$; there is thus a discontinuity, $K,-K$ at $B$, but the two values are congruent. For the anguis, $u=i K^{\prime}+$ real value $v$ : above the axis $v$ positive, viz. 
at infinity $v=0$, and at $C,=K$; below the axis $v$ negative, viz. at infinity $v=0$ and at $C,=-K$ : there thus is a discontinuity $i K^{\prime}+K, i K^{\prime}-K$ at $C$, but the two values are congruent. Observe that for points opposite to each other in regard to the axis, the arguments are, for points on the oval $u,-u$; for points 'on the anguis $i K^{\prime}+v$, $i K^{\prime}-v$ : but that we have $i K^{\prime}-v \equiv-\left(i K^{\prime}-v\right)$.

122. The pure theorem gives for three points $u_{1}, u_{2}, u_{3}$ in a line

$$
d u_{1}+d u_{2}+d u_{3}=0
$$

and thence $u_{1}+u_{2}+u_{3}=C$. The constant $C$ cannot have a determinate value (for if it had, then assuming the values of $u_{1}$ and $u_{2}$ at pleasure $u_{3}$ would have the determinate value $=C-u_{1}-u_{2}$ ), but must be given by a congruence: or, what is the same thing, assigning to $C$ any admissible value, we must instead of $u_{1}+u_{2}+u_{3}=C$, write $u_{1}+u_{2}+u_{3} \equiv C$. Taking any particular line, for instance the tangent at $A$, we have $u_{1}, u_{2}, u_{3}=0,0, i K^{\prime}$; whence $C=i K^{\prime}$; and we have $u_{1}+u_{2}+u_{3} \equiv i K^{\prime}$, viz. this is the relation between the arguments $u_{1}, u_{2}, u_{3}$ belonging to the points of intersection of the cubic with a line: in particular, for a line at right angles to the axis, we have $u_{1}, u_{2}, u_{3}=\alpha,-\alpha, i K^{\prime}$ or $=i K^{\prime}+\beta, i K^{\prime}-\beta, i K^{\prime}$ (according as the line cuts the oval or the anguis): and the congruence is in each case satisfied. But I shall in general instead of $\equiv$ use the sign $=$, understanding it as in general meaning $\equiv$, and only replacing it by this sign when for clearness it seems necessary to do so.

Writing sn $u_{1}, \operatorname{cn} u_{1}, \operatorname{dn} u_{1}=s_{1}, c_{1}, d_{1}$, and so in other cases, the condition in order that the three points may be in a line is

$$
\left|\begin{array}{lll}
s_{1}{ }^{2}, & s_{1} c_{1} d_{1}, & 1 \\
s_{2}{ }^{2}, & s_{2} c_{2} d_{2}, & 1 \\
s_{3}{ }^{2}, & s_{3} c_{3} d_{3}, & 1
\end{array}\right|=0
$$

a relation which must be satisfied when the arguments are connected by the foregoing relation $u_{1}+u_{2}+u_{3} \equiv i K^{\prime}$.

We can show from this equation alone that $s_{3}{ }^{2}$ and $s_{3} c_{3} d_{3}$ are expressible rationally in terms of $s_{1}^{2}, s_{1} c_{1} d_{1}, s_{2}^{2}, s_{2} c_{2} d_{2}$; in fact, writing therein $x_{1}, y_{1}$ in place of $s_{1}^{2}, s_{1} c_{1} d_{1}$, \&c., we thence have $x_{3}, y_{3}, 1=\lambda x_{1}+\mu x_{2}, \lambda y_{1}+\mu y_{2}, \lambda+\mu$, and substituting in

$$
y_{3}^{2}=x_{3}\left(1-x_{3}\right)\left(1-k^{2} x_{3}\right),
$$

we obtain an equation $\lambda \mu(F \lambda+G \mu)=0$, that is, $F^{\prime} \lambda+G \mu=0$, or say $\lambda, \mu=G,-F$, and thence

$$
x_{3}=s_{3}{ }^{2}=\frac{G x_{1}-F x_{2}}{G-F}, \quad y_{3}=s_{3} c_{3} d_{3}=\frac{G y_{1}-F y_{2}}{G-F} .
$$

The values of $F, G$ are easily found to be

$$
\begin{aligned}
& F=y_{1}^{2}+2 y_{1} y_{2}-x_{1}\left(1-x_{1}\right)\left(1-k^{2} x_{2}\right)-x_{1}\left(1-k^{2} x_{1}\right)\left(1-x_{2}\right)-\left(1-x_{1}\right)\left(1-k^{2} x_{1}\right) x_{2}, \\
& G=2 y_{1} y_{2}+y_{2}{ }^{2}-x_{2}\left(1-x_{2}\right)\left(1-k^{2} x_{1}\right)-x_{2}\left(1-k^{2} x_{2}\right)\left(1-x_{1}\right)-\left(1-x_{2}\right)\left(1-k^{2} x_{2}\right) x_{1},
\end{aligned}
$$


or, as these may also be written,

$$
\begin{aligned}
& F=-\left(y_{1}-y_{2}\right)^{2}+\left(x_{1}-x_{2}\right)^{2}\left\{1+k^{2}+k^{2}\left(x_{1}+x_{2}\right)\right\}+k^{2}\left(x_{1}-x_{2}\right)^{2} x_{1}, \\
& G=-\left(y_{1}-y_{2}\right)^{2}+\left(x_{1}-x_{2}\right)^{2}\left\{1+k^{2}+k^{2}\left(x_{1}+x_{2}\right)\right\}+k^{2}\left(x_{1}-x_{2}\right)^{2} x_{2},
\end{aligned}
$$

where of course $x_{1}, y_{1}, x_{2}, y_{2}$ should be replaced by their values $s_{1}{ }^{2}, s_{1} c_{1} d_{1}, s_{2}{ }^{2}, s_{2} c_{2} d_{2}$. This is, in fact, the ordinary process of finding the third point of intersection of a cubic by a line which meets it in two given points.

Writing $i K^{\prime}-u_{3}=u$, and $s, c, d$ for the sn, cn, dn of $u$, we have

whence

$$
s_{3}, c_{3}, d_{3}=-\frac{1}{k s}, \frac{i d}{k s}, \frac{i c}{s}
$$

$$
s_{3}{ }^{2}=\frac{1}{k^{2} s^{2}}, \quad s_{3} c_{3} d_{3}=\frac{c d}{k^{2} s^{3}},
$$

and the determinant equation becomes

that is,

$$
\left|\begin{array}{ccc}
s_{1}{ }^{2}, & s_{1} c_{1} d_{1}, & 1 \\
s_{2}^{2}, & s_{2} c_{2} d_{2}, & 1 \\
1, & \frac{c d}{s}, & k^{2} s^{2}
\end{array}\right|=0
$$

$$
\left(1-k^{2} s^{2} s_{2}^{2}\right) s_{1} c_{1} d_{1}-\left(1-k^{2} s^{2} s_{1}^{2}\right) s_{2} c_{2} d_{2}-\left(s_{1}^{2}-s_{2}^{2}\right) \frac{c d}{s}=0
$$

corresponding to the relation $u=u_{1}+u_{2}$ of the arguments. This is easily verified: we have

$$
s_{1}=\frac{s c_{2} d_{2}-s_{2} c d}{1-k^{2} s^{2} s_{2}{ }^{2}}, \quad s_{2}=\frac{s c_{1} d_{1}-s_{1} c d}{1-k^{2} s^{2} s_{1}^{2}}, \quad s=\frac{s_{1}^{2}-s_{2}^{2}}{s_{1} c_{2} d_{2}-s_{2} c_{1} d_{1}} ;
$$

the equation thus becomes

$$
\left(s c_{2} d_{2}-s_{2} c d\right) c_{1} d_{1}-\left(s c_{1} d_{1}-s_{1} c d\right) c_{2} d_{2}-\left(s_{1}^{2}-s_{2}^{2}\right) \frac{c d}{s}=0,
$$

that is,

which is right.

$$
c d\left\{-s_{2} c_{1} d_{1}+s_{1} c_{2} d_{2}-\frac{s_{1}^{2}-s_{2}^{2}}{s}\right\}=0
$$

\section{The Four Tangents from a Point of the Cubic.}

123. Suppose that the line is a tangent to the cubic, say the line touches the cubic at the point $u$, and again meets it at the point $w$ : then instead of $u_{1}, u_{2}, u_{3}$ we have $u, u, w$ : and the relation becomes $2 u+w \equiv i K^{\prime}$.

Here $u$ being given, $w$ is uniquely determined: viz. given the argument $u$ of the point of contact, we have a unique value for the argument $w$ of the tangential. But given $w$, we have $2 u=i K^{\prime}-w$; and we have thus for $u$ the four values

$$
\frac{1}{2}\left(i K^{\prime}-w\right), \quad \text { Do. }+K, \quad \text { Do. }+i K^{\prime}, \quad \text { Do. }+K+i K^{\prime},
$$

corresponding to the four tangents which can be drawn from the point $w$ to the cubic. 
The tangents are real for a point of the anguis, and for such a point we may write $w=i K^{\prime}+v$, where $v$ is real and included between the values $\pm K$; the corresponding values of $u$ are

$$
u_{1}=-\frac{1}{2} v, \quad u_{2}=-\frac{1}{2} v+K, \quad u_{3}=-\frac{1}{2} v+i K^{\prime}, \quad u_{4}=-\frac{1}{2} v+K+i K^{\prime}: \cdots
$$

the first and second of these belong to tangents to the oval, the third and fourth to tangents to the anguis. We may further distinguish a tangent according as it passes between or does not pass between the vertices $B$ and $C$ : say in the former case it is intermediate, and in the latter case extramediate: and we then see that, for the tangents from the point $i K^{\prime}+v$ of anguis,

$$
\begin{array}{ll}
u=-\frac{1}{2} v & \text { for intermediate to oval, } \\
u=-\frac{1}{2} v+K & \text { " extramediate to oval, } \\
u=-\frac{1}{2} v+i K^{\prime} & \text { " extramediate to anguis, } \\
u=-\frac{1}{2} v+K+i K^{\prime} & \text { " intermediate to anguis. }
\end{array}
$$

124. We may make a corresponding division of the real lines which meet the curve in three real points: any such line meets the oval twice (and then of course the anguis once), or else it meets the anguis three times: and taking the arguments to be $u_{1}, u_{2}, u_{3}$, we have

$$
\begin{aligned}
\frac{1}{2}\left(u_{1}+u_{2}+u_{3}\right) & =\frac{1}{2} i K^{\prime} \quad \text { for intermediate line meeting oval twice, } \\
& =\frac{1}{2} i K^{\prime}+K \quad, \quad \text { extramediate line, Do., } \\
& =-\frac{1}{2} i K^{\prime} \quad, \quad \text { extramediate line meeting anguis three times, } \\
& =-\frac{1}{2} i K^{\prime}+K \quad, \quad \text { intermediate line, Do. }
\end{aligned}
$$

125. Returning to the tangents, the point $i K^{\prime}+v$ may be an inflexion: we have then the point of contact of the intermediate tangent to the anguis coinciding with the point $i K^{\prime}+v$; viz. $i K^{\prime}+v \equiv-\frac{1}{2} v+K+i K^{\prime}$, or say $=-\frac{1}{2} v \pm K+i K^{\prime}$ : that is, $v= \pm \frac{2}{3} K$; or $i K^{\prime}+\frac{2}{3} K$ and $i K^{\prime}-\frac{2}{3} K$ are the arguments for the real points of inflexion, above and below the axis respectively.

126. Write for a moment the equation in the form $y^{2}=B x+C x^{2}+D x^{3}$; then if $(\alpha, \beta)$ be a point on the curve $\left(\beta^{2}=B \alpha+C \alpha^{2}+D \alpha^{3}\right)$, and we consider the intersections of the curve with the line $y-\beta=m(x-\alpha)$, we find for the remaining two intersections

$$
B+C(x+\alpha)+D\left(x^{2}+\alpha x+\alpha^{2}\right)=2 m \beta+m^{2}(x-\alpha) .
$$

If the line be a tangent, this will be satisfied by $x-\alpha$; the condition for this is $2 m \beta=B+2 C \alpha+3 D \alpha^{2}$, and supposing this satisfied, then throwing out the factor $x-\alpha$ we obtain $C+D(x+2 \alpha)=m^{2}$, giving $D x=m^{2}-C-2 D \alpha$ for the coordinate $x$ of the tangential of the point $(\alpha, \beta)$.

In the case of an inflexion, $x=\alpha$; and we have

$$
m^{2}=C+3 D \alpha,=\frac{\left(B+2 C \alpha+3 D \alpha^{2}\right)^{2}}{4\left(B \alpha+C \alpha^{2}+D \alpha^{3}\right)},
$$


giving for $\alpha$ the equation

$$
3 D^{2} \alpha^{4}+4 C D \alpha^{3}+6 B D \alpha-B^{2}=0,
$$

or for $B, C, D$ writing their values $1,-\left(1+k^{2}\right), k^{2}$, this is

$$
3 k^{4} \alpha^{4}-4 k^{2}\left(1+k^{2}\right) \alpha^{3}+6 k^{2} \alpha^{2}-1=0
$$

for the $x$-coordinate of the inflexion. There is one negative root, and one or three positive roots; but only one positive root giving a real value of $\beta$, and corresponding hereto we have the two real inflexions on the anguis.

It should be possible, from the formulæ of No. 122, writing therein $\left(x_{2}, y_{2}\right)=\left(x_{1}, y_{1}\right)$, to arrive at the foregoing result, say $D x_{3}=m^{2}-C-2 D x_{1}$; but the functions $F$ and $G$ present themselves in vanishing forms, and the reduction is not immediate.

127. The general condition for an inflexion is $3 u \equiv i K^{\prime}:$ the nine inflexions thus are $u=i K^{\prime}$, inflexion at infinity, $u=i K^{\prime} \pm \frac{2}{3} K$, the two real inflexions, and besides $u= \pm \frac{1}{3} i K^{\prime}, u= \pm \frac{1}{3} i K^{\prime} \pm \frac{2}{3} K$, six imaginary inflexions.

\section{The Sextactic Points.}

128. The vertices $A, B, C$ are each of them a sextactic point: in fact, writing the equation in the form $y^{2}=x-\left(1+k^{2}\right) x^{2}+k^{2} x^{3}$, we see at once that the conic $y^{2}=x-\left(1+k^{2}\right) x^{2}$ meets the curve in the point $A$ counting six times: and there is obviously a like proof for the vertices $B$ and $C$ respectively. Hence, for the six intersections with any conic whatever, we have the condition

$$
u_{1}+u_{2}+u_{3}+u_{4}+u_{5}+u_{6} \equiv 0:
$$

and for the sextactic points we have the condition $6 u \equiv 0$. This gives the 36 points $u=\frac{1}{6}\left(2 m K+2 m^{\prime} i K^{\prime}\right)$ or say $=\frac{1}{3}\left(m K+m^{\prime} i K^{\prime}\right), m=0, \pm 1, \pm 2,3, m^{\prime}=0, \pm 1, \pm 2,3:$ but among these are included the 9 inflexions (each of these being an improper sextactic point, the conic becoming the tangent taken twice) and there remain 27 points: among these are included the three vertices $\left(u=0, K, i K^{\prime}+K\right)$, points of contact with the tangents from the inflexion at infinity; and of the remaining 24 points 6 are real, viz. these are the points $u= \pm \frac{1}{3} K, \pm \frac{2}{3} K$ on the oval, and the points $i K^{\prime} \pm \frac{1}{3} K$ on the anguis: these are, in fact, the points of contact of the tangents from the real inflexions, viz. the three tangents from the inflexion $i K^{\prime}+\frac{2}{3} K$ touch the oval in the points $\frac{2}{3} K,-\frac{1}{3} K$, and the anguis in the point $i K^{\prime}-\frac{1}{3} K$; the three tangents from the inflexion $i K^{\prime}-\frac{2}{3} K$ touch the oval in the points $-\frac{2}{3} K, \frac{1}{3} K$, and the anguis in the point $i K^{\prime}+\frac{1}{3} K$.

\section{Formulce Relating to the Tangents from the Vertices.}

129. I annex some formulæ relating to the tangents to the curve from the vertices $A, B, C$ respectively. We have from each vertex four tangents say $\rho=0, \sigma=0$, symmetrically situate in regard to the axis, and $\rho^{\prime}=0, \sigma^{\prime}=0$, symmetrically situate in regard to the axis: the line joining the points of contact of $\rho, \sigma$ is a line $\tau=0$ at 
right angles to the axis, and that joining the points of contact of $\rho^{\prime}, \sigma^{\prime}$ is a line $\tau^{\prime}=0$ at right angles to the axis.

Vertex A, Tangents Imaginary.

$$
\begin{array}{ll}
\rho, \sigma, \tau=y-i(1+k) x, y+i(1+k) x, \quad k x+1 ; & x=-\frac{1}{k}, y=\mp \frac{i(1+k)}{k}, \\
\rho^{\prime}, \sigma^{\prime}, \tau^{\prime}=y-i(1-k) x, y+i(1-k) x,-k x+1 ; & x=\frac{1}{k}, y= \pm \frac{i(1-k)}{k} ;
\end{array}
$$

the equation of the curve is

$$
f,=y^{2}-x(1-x)\left(1-k^{2} x\right),=\rho \sigma-x \tau^{2},=\rho^{\prime} \sigma^{\prime}-x \tau^{\prime 2},=0 .
$$

Vertex B, Tangents Imaginary.

Contacts.

$\rho, \sigma, \tau=y-\left(k-i k^{\prime}\right)(1-x), y+\left(k+i k^{\prime}\right)(1-x), k x-\left(k-i k^{\prime}\right) ; \quad x=1-\frac{i k^{\prime}}{k}, y=\mp \frac{i k^{\prime}}{k}\left(k+i k^{\prime}\right)$, $\rho^{\prime}, \sigma^{\prime}, \tau^{\prime}=y-\left(k-i k^{\prime}\right)(1-x), y+\left(k-i k^{\prime}\right)(1-x), k x-\left(k+i k^{\prime}\right) ; \quad x=1+\frac{i k^{\prime}}{k}, y= \pm \frac{i k^{\prime}}{k}\left(k-i k^{\prime}\right) ;$ the equation of the curve is

$$
f=\rho \sigma+(1-x) \tau^{2},=\rho^{\prime} \sigma^{\prime}+(1-x) \tau^{\prime 2},=0 .
$$

Vertex C, Tangents Real.

Contacts.

$$
\begin{aligned}
& \rho, \sigma, \tau=y-\frac{1}{1+k^{\prime}}\left(1-k^{2} x\right), y+\frac{1}{1+k^{\prime}}\left(1-k^{2} x\right), \quad x-\frac{1}{1+k^{\prime}} ; \quad x=\frac{1}{1+k^{\prime}}, y=\frac{ \pm k^{\prime}}{1+k^{\prime}}, \\
& \rho^{\prime}, \sigma^{\prime}, \tau^{\prime}=y-\frac{1}{1-k^{\prime}}\left(1-k^{2} x\right), y+\frac{1}{1-k^{\prime}}\left(1-k^{2} x\right), \quad x-\frac{1}{1-k^{\prime}} ; \quad x=\frac{1}{1-k^{\prime}}, y=\frac{ \pm k^{\prime}}{1-k^{\prime}}
\end{aligned}
$$

the equation of the curve is

$$
f,=\rho \sigma-\left(1-k^{2} x\right) \tau,=\rho^{\prime} \sigma^{\prime}-\left(1-k^{2} x\right) \tau^{\prime},=0 .
$$

130. These linear functions $\rho, \sigma$, \&c., considering therein $x, y$ as denoting $\mathrm{sn}^{2} u$, sn $u$ cn $u \operatorname{dn} u$, respectively present themselves as the numerators and the denominators of some formulæ given No. 105 of my Elliptic Functions (1876), see p. 76 : viz. we have

which is

$$
\operatorname{sn}^{2}\left(u+\frac{1}{2} K\right)=\frac{1}{1+k^{\prime}} \frac{1-k^{2} x+\left(1+k^{\prime}\right) y}{1-k^{2} x+\left(1-k^{\prime}\right) y},
$$

$$
\begin{aligned}
& =\frac{1}{1-k^{\prime}} \frac{y+\frac{1}{1+k^{\prime}}\left(1-k^{2} x\right)}{y+\frac{1}{1-k^{\prime}}\left(1-k^{2} x\right)},=\frac{1}{1-k^{\prime}} \frac{\sigma}{\sigma^{\prime}}, \quad(\text { vertex C)} ; \\
\operatorname{sn}^{2}\left(u+\frac{1}{2} i K^{\prime}\right) & =\frac{1}{k} \frac{(1+k) x+i y}{(1+k) x-i y},
\end{aligned}
$$

which is

$$
=-\frac{1}{k} \frac{y-i(1+k) x}{y+i(1+k) x}, \quad=-\frac{1}{k} \frac{\rho}{\sigma}, \quad(\text { vertex } \mathrm{A}) \text {; }
$$

and

$$
\operatorname{sn}^{2}\left(u+\frac{1}{2} K+\frac{1}{2} i K^{\prime}\right)=\frac{k+i k^{\prime}}{k} \frac{1-x+(k-i k) y}{1-x+(k+i k) y},
$$

c. XII, 
which is

$$
=\frac{k-i k^{\prime}}{k} \frac{y+\left(k+i k^{\prime}\right)(1-x)}{y+\left(k-i k^{\prime}\right)(1-x)}, \quad=\frac{k-i k^{\prime}}{k} \frac{\sigma}{\sigma^{\prime}},(\text { vertex B }) .
$$

Observe here that, in the second formula, we have a pair of tangents $\rho, \sigma$ which belong to a chord $\tau$ through the inflexion at $\infty$; but in the first and third formulæ we have tangents $\sigma, \sigma^{\prime}$ not forming such a pair. This is as it should be, for the zero and infinity of $\operatorname{sn}^{2}\left(u+\frac{1}{2} i K^{\prime}\right)$ are $u=-\frac{1}{2} i K^{\prime}, u=\frac{1}{2} i K^{\prime}$, which belong to points in lineâ with the inflexion at infinity: but for $\operatorname{sn}^{2}\left(u+\frac{1}{2} K\right)$, the zero is $u=-\frac{1}{2} K$, and the infinity is $u=i K^{\prime}-\frac{1}{2} K$, which do not belong to points in lineâ with the inflexion at infinity: and the like for $\operatorname{sn}^{2}\left(u+\frac{1}{2} K+\frac{1}{2} i K^{\prime}\right)$.

Fixed Curve a Quartic in Space, the Quadri-quadric Curve $y^{2}=1-x^{2}, z^{2}=1-k^{2} x^{2}$. Art. Nos. 131 to 135.

131. It is assumed that $k^{2}$ is real, positive, and less than unity: the curve may be regarded as the intersection of the two cylinders

$$
x^{2}+y^{2}=1, \quad k^{2} x^{2}+z^{2}=1 ;
$$

but there is through it a third cylinder $y^{2}-k^{2} z^{2}=k^{\prime 2}$. The cylinder $k^{2} x^{2}+z^{2}=1$, or say the horizontal cylinder, has for its section an ellipse axes $\frac{1}{k}$ and 1 respectively: and it is pierced by the cylinder $x^{2}+y^{2}=1$, or say the vertical cylinder, in two detached ovals (of double curvature) lying on opposite sides of the plane of $x y$ : only the upper oval $A B A^{\prime} B^{\prime}$ is shown in the figure.

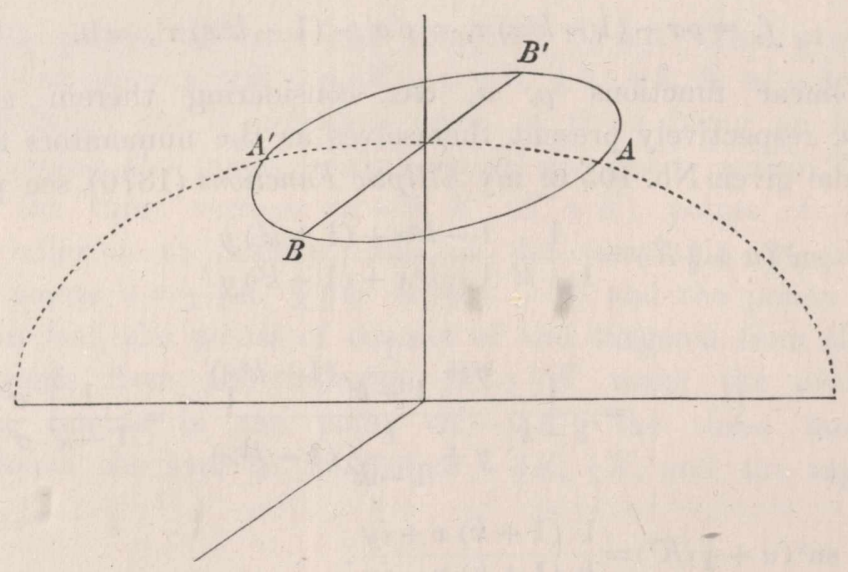

Each of the vertices $A, A^{\prime}, B, B^{\prime}$ is an inflexion*, viz. a point such that the osculating plane at that point meets the curve in the point counting four times. We may consider two generating lines of the horizontal cylinder, each meeting the oval in two points; the plane through the generating lines meets the curve in these

* There are in all 16 inflexions: 4 in each of the planes $x=0, y=0, z=0$, and 4 in the plane infinity. 
four points, and when the generating lines come each of them to coincide with the tangent at $A$, we have the osculating plane meeting the curve in the point $A$ counting four times. The like reasoning, with two generating lines of the third cylinder, shows that the vertex $B$ is an inflexion.

132. The equations are satisfied by writing therein $x, y, z=\operatorname{sn} u$, en $u, \operatorname{dn} u$ : the periods are here $4 K, 4 i K^{\prime}$ : hence, at a given point on the curve, the argument is not $u=\mathrm{a}$ determinate value $u_{0}$, but it may equally well be taken to be $=u_{0}+4 m K+4 m^{\prime} i K^{\prime}$, where $m$ and $m^{\prime}$ are any positive or negative integers: we express this by $u \equiv u_{0}$, or say $u$ congruent to $u_{0}$. For the upper oval, $u$ may be taken to be real, and to be $=0$ at $B$, positive for the half oval $B A B^{\prime}$, and negative for the half oval $B A^{\prime} B^{\prime}$; having the values $+K,-K$ at $A$ and $A^{\prime}$ respectively, and the discontinuity $2 K,-2 K$ at $B^{\prime}$, these two values being congruent. For the lower oval, we have $u=2 i K^{\prime}+$ real value $v$.

For the intersections of the curve with a plane, we have

$$
d u_{1}+d u_{2}+d u_{3}+d u_{4}=0 ; \text { whence } u_{1}+u_{2}+u_{3}+u_{4}=C ;
$$

and by taking the plane to be the osculating plane at $B$, we find 0 as a value of the constant, and the relation thus is $u_{1}+u_{2}+u_{3}+u_{4} \equiv 0$. Writing as before sn $u_{1}$, cn $u_{1}, \operatorname{dn} u_{1},=s_{1}, c_{1}, d_{1}$ and so in other cases, the relation between the elliptic functions is

$$
\left|\begin{array}{llll}
s_{1}, & c_{1}, & d_{1}, & 1 \\
s_{2}, & c_{2}, & d_{2}, & 1 \\
s_{3}, & c_{3}, & d_{3}, & 1 \\
s_{4}, & c_{4}, & d_{4}, & 1
\end{array}\right|=0
$$

It is important to remark that, given three of the points, the fourth point is determined uniquely: that is, the equation really gives $s_{4}, c_{4}, d_{4}$, each as a rational function of the $s_{1}, c_{1}, d_{1}, s_{2}, c_{2}, d_{2}, s_{3}, c_{3}, d_{3}$.

In fact, we may write $s_{4}=\lambda_{1} s_{1}+\lambda_{2} s_{2}+\lambda_{3} s_{3}$, and similarly for $c_{4}$ and $d_{4}$, and $1=\lambda_{1}+\lambda_{2}+\lambda_{3}$ : substituting in $s_{4}{ }^{2}+c_{4}{ }^{2}-1=0, k^{2} s_{4}{ }^{2}+d_{4}{ }^{2}-1=0$, we have

where

$$
\begin{aligned}
& X_{23} \lambda_{2} \lambda_{3}+X_{31} \lambda_{3} \lambda_{1}+X_{12} \lambda_{1} \lambda_{2}=0 \\
& Y_{23},+Y_{31} \quad+Y_{12} \quad,=0,
\end{aligned}
$$

we thence have

$$
X_{12}=s_{1} s_{2}+c_{1} c_{2}-1, \quad Y_{12}=k^{2} s_{1} s_{2}+d_{1} d_{2}-1, \quad \text { \&c. ; }
$$

$$
\begin{aligned}
& \lambda_{2} \lambda_{3}: \lambda_{3} \lambda_{1}: \lambda_{1} \lambda_{2}=X_{31} Y_{12}-X_{12} Y_{31}: X_{12} Y_{23}-X_{23} Y_{12}: X_{23} Y_{31}-X_{31} Y_{23} \\
& =A_{1} \quad: \quad A_{2} \quad: \quad A_{3} \text {, suppose ; }
\end{aligned}
$$

that is,

$$
\lambda_{1}: \lambda_{2}: \lambda_{3}=A_{2} A_{3}: A_{3} A_{1}: A_{1} A_{2}
$$

and consequently

$$
\begin{aligned}
& s_{4},=- \text { sn }\left(u_{1}+u_{2}+u_{3}\right),=A_{2} A_{3} s_{1}+A_{3} A_{1} s_{2}+A_{1} A_{2} s_{3} \div\left(A_{2} A_{3}+A_{3} A_{1}+A_{1} A_{2}\right), \\
& c_{4},=\operatorname{cn}(\quad)=, c_{1}+, c_{2}+, c_{3} \div( \\
& d_{4},=\operatorname{dn}(, \quad)=, d_{1}+, d_{2}+, d_{3} \div(
\end{aligned}
$$


which are the required expressions. If $u_{3}=0$, and consequently $s_{3}, c_{3}, d_{3}=0,1,1$, the resulting expressions give the $\mathrm{sn}$, en, and $\mathrm{dn}$ of $u_{1}+u_{2}$; but the expressions are in a very complicated form, not easily identifiable with the ordinary ones.

133. The determinant equation may be written

$$
\begin{aligned}
& \left(s_{1}-s_{2}\right)\left(c_{3} d_{4}-c_{4} d_{3}\right)+\left(s_{3}-s_{4}\right)\left(c_{1} d_{2}-c_{2} d_{1}\right) \\
+ & \left(c_{1}-c_{2}\right)\left(d_{3} s_{4}-d_{4} s_{3}\right)+\left(c_{3}-c_{4}\right)\left(d_{1} s_{2}-d_{2} s_{1}\right) \\
+ & \left(d_{1}-d_{2}\right)\left(s_{3} c_{4}-s_{4} c_{3}\right)+\left(d_{3}-d_{4}\right)\left(s_{1} c_{2}-s_{2} c_{1}\right)=0
\end{aligned}
$$

and, in fact, each of the three lines is separately $=0$. This appears from the following three formulæ:-

$$
\begin{gathered}
\frac{\operatorname{sn}\left(u_{1}+u_{2}\right)}{\operatorname{cn}\left(u_{1}+u_{2}\right)-\operatorname{dn}\left(u_{1}+u_{2}\right)}=\frac{s_{1}-s_{2}}{c_{1} d_{2}-c_{2} d_{1}}, \\
\frac{\operatorname{sn}\left(u_{1}+u_{2}\right)}{\operatorname{cn}\left(u_{1}+u_{2}\right)+1}=\frac{c_{1}-c_{2}}{d_{1} s_{2}-d_{2} s_{1}}, \\
\frac{\operatorname{sn}\left(u_{1}+u_{2}\right)}{\operatorname{dn}\left(u_{1}+u_{2}\right)+1}=\frac{-\frac{1}{k^{2}}\left(d_{1}-d_{2}\right)}{s_{1} c_{2}-s_{2} c_{1}},
\end{gathered}
$$

which are themselves at once deducible from the formulæ

$$
\begin{array}{lll}
\text { sn }\left(u_{1}+u_{2}\right)=s_{1}{ }^{2}-s_{2}{ }^{2},=-\left(c_{1}{ }^{2}-c_{2}{ }^{2}\right),=-\frac{1}{k^{2}}\left(d_{1}{ }^{2}-d_{2}{ }^{2}\right) & \div\left(s_{1} c_{2} d_{2}-s_{2} c_{1} d_{1}\right), \\
\text { cn }\left(u_{1}+u_{2}\right)=s_{1} c_{1} d_{2}-s_{2} c_{2} d_{1} & \div & \text { Do. } \\
\operatorname{dn}\left(u_{1}+u_{2}\right)=s_{1} d_{1} c_{2}-s_{2} d_{2} c_{1} & \div & \text { Do. }
\end{array}
$$

In fact, the numerators of $\operatorname{cn}\left(u_{1}+u_{2}\right)+\operatorname{dn}\left(u_{1}+u_{2}\right)$, cn $\left(u_{1}+u_{2}\right)+1, \operatorname{dn}\left(u_{1}+u_{2}\right)+1$, thus become $=\left(s_{1}+s_{2}\right)\left(c_{1} d_{2}-c_{2} d_{1}\right),-\left(c_{1}+c_{2}\right)\left(d_{1} s_{2}-d_{2} s_{1}\right),\left(d_{1}+d_{2}\right)\left(s_{1} c_{2}-s_{2} c_{1}\right)$, respectively: so that, taking the numerator of $\operatorname{sn}\left(u_{1}+u_{2}\right)$ under its three forms successively, we have by division the formulæ in question.

134. The above three equations, putting therein $u_{1}+u_{2}=-2 u_{3}$ and reducing the functions of $2 u_{3}$, become

$$
\begin{aligned}
\frac{1}{k^{\prime 2}} \frac{c_{3} d_{3}}{s_{3}} & =\frac{s_{1}-s_{2}}{c_{1} d_{2}-c_{2} d_{1}}, \text { giving }-k^{2} s_{3}{ }^{2}=\frac{\left(c_{1}-c_{2}\right)\left(d_{1}-d_{2}\right)}{\left(d_{1} s_{2}-d_{2} s_{1}\right)\left(s_{1} c_{2}-s_{2} c_{1}\right)}, \\
-\frac{s_{3} d_{3}}{c_{3}} & =\frac{c_{1}-c_{2}}{d_{1} s_{2}-d_{2} s_{1}}, \quad \# \quad{\frac{k}{k^{\prime 2}}}^{2} c_{3}{ }^{2}=\frac{\left(d_{1}-d_{2}\right)\left(s_{1}-s_{2}\right)}{\left(s_{1} c_{2}-s_{2} c_{1}\right)\left(c_{1} d_{2}-c_{2} d_{1}\right)}, \\
\frac{k^{2} s_{3} c_{3}}{d_{3}} & =\frac{d_{1}-d_{2}}{s_{1} c_{2}-s_{2} c_{1}}, \quad, \quad-\frac{1}{k^{\prime 2}} d_{3}{ }^{2}=\frac{\left(s_{1}-s_{2}\right)\left(c_{1}-c_{2}\right)}{\left(c_{1} d_{2}-c_{2} d_{1}\right)\left(d_{1} s_{2}-d_{2} s_{1}\right)},
\end{aligned}
$$

equations which must of course give each of them the same value for ${s_{3}}^{2}$ : the equations belong to the relation $u_{1}+u_{2}+2 u_{3}=0$, viz. $\left(s_{3}, c_{3}, d_{3}\right)$ are the coordinates of a point of contact of the tangent plane drawn through the two given points $\left(s_{1}, c_{1}, d_{1}\right)$ and $\left(s_{2}, c_{2}, d_{2}\right)$ of the curve. 
135. Write

$$
\begin{aligned}
& a, b, c, f, g, h=s_{1}-s_{2}, c_{1}-c_{2}, d_{1}-d_{2}, c_{1} d_{2}-c_{2} d_{1}, d_{1} s_{2}-d_{2} s_{1}, s_{1} c_{2}-s_{2} c_{1}, \\
& a^{\prime}, b^{\prime}, c^{\prime}, f^{\prime}, g^{\prime}, h^{\prime}=s_{3}-s_{4}, c_{3}-c_{4}, d_{3}-d_{4}, c_{3} d_{4}-c_{4} d_{3}, d_{3} s_{4}-d_{4} s_{3}, s_{3} c_{4}-s_{4} c_{3},
\end{aligned}
$$

so that $(a, b, c, f, g, h)$ are the six coordinates of the line 12, and $a^{\prime}, b^{\prime}, c^{\prime}, f^{\prime}, g^{\prime}, h^{\prime}$ are the six coordinates of the line 34. The determinant equation is nothing else than the condition of the intersection of the two lines, viz. this is

$$
a f^{\prime}+a^{\prime} f+b g^{\prime}+b^{\prime} g+c h^{\prime}+c^{\prime} h=0 \text {. }
$$

By what precedes, it appears that not only is this so: but that we have separately $a f^{\prime}+a^{\prime} f=0, b g^{\prime}+b^{\prime} g=0, \quad c h^{\prime}+c^{\prime} h=0$, viz. these three equations are satisfied by the coordinates of the lines 12 and 34, which join in pairs the intersections 1,2 and 3,4 of the quadri-quadric curve by a plane. But this is a geometrical property depending only on the four points being in a plane: and it is thus a result of Abel's theorem that, when the arguments are such that

$$
u_{1}+u_{2}+u_{3}+u_{4}=0,
$$

then not only the original equation, but each of the three equations, holds good.

The Cubic Curve $x y^{2}-2 y+\left(1+k^{2}\right) x-k^{2} x^{3}=0$. Art. Nos. 136 and 137.

136. Writing the equation in the form

$$
(x y-1)^{2}=\left(1-x^{2}\right)\left(1-k^{2} x^{2}\right) \text {, or say } x y-1=-\sqrt{1-x^{2} .1-k^{2} x^{2}},
$$

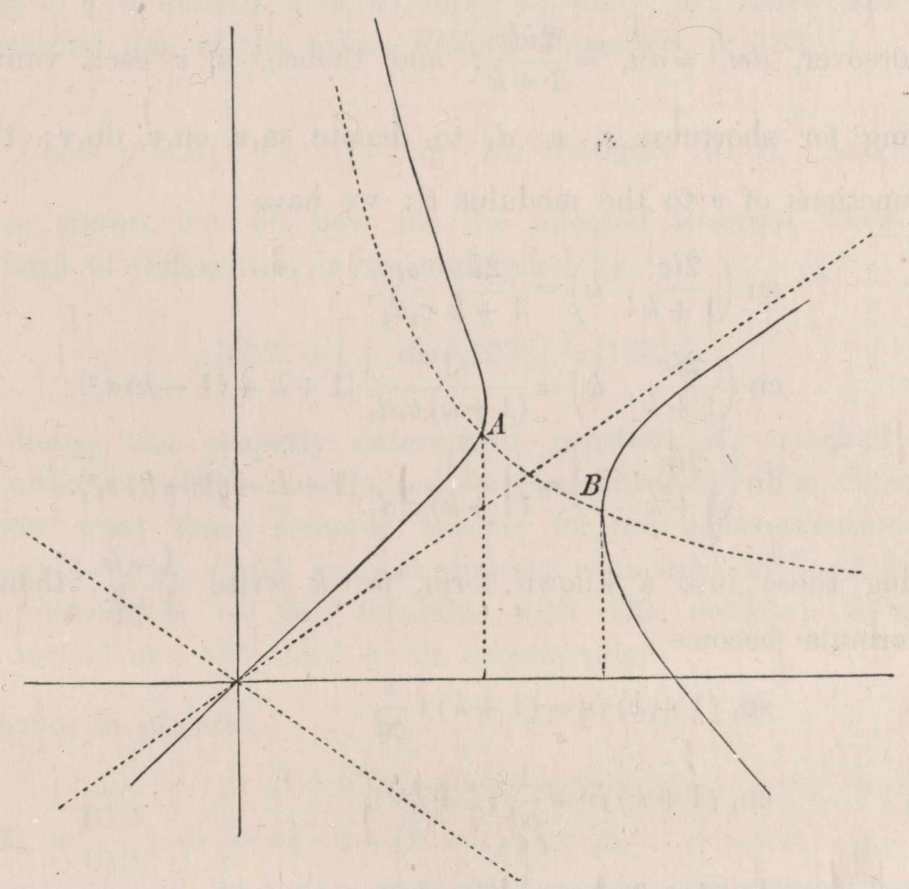

we see that the general form is as shown in the figure: the real portions of the 
curve lie between the values $x=-\infty,-\frac{1}{k} ;-1,+1$; and $\frac{1}{k}, \infty$. The curve may be made to depend on elliptic functions in two different ways: we may write

$$
\begin{aligned}
& x=\operatorname{sn} u \quad, \quad=\frac{2 i}{1+k} \frac{\operatorname{sn}_{1} v}{\operatorname{cn}_{1} v \operatorname{dn}_{1} v}, \\
& y=\frac{1-\operatorname{cn} u \operatorname{dn} u}{\operatorname{sn} u},=\frac{i}{1+k} \frac{\operatorname{sn}_{1} v}{\operatorname{cn}_{1} v \operatorname{dn}_{1} v}\left\{1+k^{2}-(1-k)^{2} \operatorname{sn}_{1}^{2} v\right\},
\end{aligned}
$$

where the functions $\mathrm{sn}, \mathrm{cn}, \mathrm{dn}$ belong to the modulus $k$, and the functions $\mathrm{sn}_{1}, \mathrm{cn}_{1}, \mathrm{dn}_{1}$ to the modulus $\theta,=\frac{1-k}{1+k}$. The first mode is obvious; as to the second, observe that the formulæ give

whence

$$
\begin{aligned}
& y-k x=\frac{i}{1+k} \frac{\operatorname{sn}_{1} v}{\operatorname{cn}_{1} v \operatorname{dn}_{1} v}(1-k)^{2} \mathrm{cn}_{1}^{2} v,=\frac{i(1-k)^{2}}{1+k} \frac{\operatorname{sn}_{1} v \mathrm{cn}_{1} v}{\mathrm{dn}_{1} v} \\
& y+k x=\frac{i}{1+k} \frac{\operatorname{sn}_{1} v}{\operatorname{cn}_{1} v \operatorname{dn}_{1} v}(1+k)^{2} \mathrm{dn}_{1}^{2} v,=i(1+k) \frac{\mathrm{sn}_{1} v \mathrm{dn}_{1} v}{\mathrm{cn}_{1} v}
\end{aligned}
$$

$$
y^{2}-k^{2} x^{2}=-(1-k)^{2} \operatorname{sn}_{1}^{2} v
$$

and therefore

$$
x\left(y^{2}-k^{2} x^{2}\right)=\frac{-2 i(1-k)^{2}}{1+k} \frac{\operatorname{sn}_{1}^{3} v}{\operatorname{cn}_{1} v \operatorname{dn}_{1} v},
$$

which is also the value of $2 y-\left(1+k^{2}\right) x$, as it should be.

We find, moreover, $d \omega,=d u,=\frac{2 i d v}{1+k}$; and thence, $u, v$ each vanishing together, $u=\frac{2 i v}{1+k}$. Writing for shortness $s_{1}, c_{1}, d_{1}$ to denote $\mathrm{sn}_{1} v, \mathrm{cn}_{1} v, \mathrm{dn}_{1} v$ : that is, $s_{1}, c_{1}, d_{1}$ are the elliptic functions of $v$ to the modulus $\theta$ : we have

$$
\begin{aligned}
& \operatorname{sn}\left(\frac{2 i v}{1+k}, k\right)=\frac{2 i}{1+k} \frac{s_{1}}{c_{1} d_{1}} \\
& \text { cn }\left(\frac{2 i v}{1+k}, k\right)=\frac{1}{(1+k) c_{1} d .}\left\{1+k+(1-k) s_{1}^{2}\right\} \\
& \operatorname{dn}\left(\frac{2 i v}{1+k}, k\right)=\frac{1}{(1+k) c_{1} d_{1}}\left\{1+k-(1-k) s_{1}^{2}\right\}
\end{aligned}
$$

137. To bring these into a known form, for $k$ write $\frac{1-k}{1+k}$, then $\theta$ is changed into $k$, and the formulæ become

$$
\begin{aligned}
& \operatorname{sn}_{1}(1+k) i v=(1+k) i \frac{s}{c d} \\
& \mathrm{cn}_{1}(1+k) i v=\frac{1}{c d}\left(1+k s^{2}\right) \\
& \operatorname{dn}_{1}(1+k) i v=\frac{1}{c d}\left(1-k s^{2}\right),
\end{aligned}
$$


where the $\mathrm{sn}_{1}, \mathrm{cn}_{1}, \mathrm{dn}_{1}$ refer to the modulus $\theta$, and $s, c, d$ denote $\operatorname{sn} v, \operatorname{cn} v, \operatorname{dn} v$, modulus $k$.

But from the formulæ, p. 63, of my Elliptic Functions,

$$
\operatorname{sn}(i u, k), \operatorname{cn}(i u, k), \operatorname{dn}(i u, k)=\frac{i \operatorname{sn}\left(u, k^{\prime}\right)}{\operatorname{cn}\left(u, k^{\prime}\right)}, \frac{1}{\operatorname{cn}\left(u, k^{\prime}\right)}, \frac{\operatorname{dn}\left(u, k^{\prime}\right)}{\operatorname{cn}\left(u, k^{\prime}\right)},
$$

and herein for $u$ writing $(1+k) v$, and for $k$ writing $\theta,=\frac{1-k}{1+k}$, whence $k^{\prime}$ becomes $\frac{2 \sqrt{k}}{1+k},=\gamma$, and $\gamma^{\prime}=\frac{1-k}{1+k}$, we have

$\mathrm{sn}_{1}(1+k) i v, \quad \mathrm{cn}_{1}(1+k) i v, \mathrm{dn}_{1}(1+k) i v=\frac{i \mathrm{sn}(1+k v, \gamma)}{\mathrm{cn}(\overline{1+k v}, \gamma)}, \frac{1}{\mathrm{cn}(1+k v, \gamma)}, \frac{\operatorname{dn}(\overline{1+k v}, \gamma)}{\mathrm{cn}(\overline{1+k v}, \gamma)}$ and the formulæ above obtained are

$$
\begin{aligned}
& \frac{\text { sn }(1+k v, \gamma)}{\operatorname{cn}(1+k v, \gamma)}=(1+k) \frac{s}{c d}, \quad \text { giving sn }(1+k v, \gamma)=\frac{(1+k) s}{1+k s^{2}} \\
& \frac{1}{\operatorname{cn}(\overline{1+k} v, \gamma)}=\frac{1}{c d}\left(1+k s^{2}\right), \quad " \quad \operatorname{cn}(1+k v, \gamma)=\frac{c d}{1+k s^{2}} \\
& \frac{\operatorname{dn}(1+k v, \gamma)}{\operatorname{cn}(\overline{1+k} v, \gamma)}=\frac{1}{c d}\left(1-k s^{2}\right), \quad, \quad \operatorname{dn}(1+k v, \gamma)=\frac{1-k s^{2}}{1+k s^{2}},
\end{aligned}
$$

where, as before, $s, c, d$ denote $\operatorname{sn}(v, k), \operatorname{cn}(v, k), \operatorname{dn}(v, k)$ : these last are, in fact, the formulæ of the second line of the table, Elliptic Functions, p. 183.

Fixed Curve the Cubic $y^{2}=x(1-x)\left(1-k^{2} x\right)$ : the Function $\left\{01^{\prime} \theta\right\}$. Art. Nos. 138 to 142.

138. It was shown, No. 65, how for the affected theorem, when the fixed curve is a cubic, the form of differential is $d \omega$ multiplied by

$$
\{012\}+\left[\int_{2}^{1} d \omega \partial_{3}\{036\}-\{123\}\right]
$$

the last term being the properly determined constant $K$, attached to the variable term $\{012\}$, in order to obtain a standard form of integral. The object of the present articles is to show what these formulæ become for the before-mentioned form of cubic curve $y^{2}=x(1-x)\left(1-k^{2} x\right)$, which is most directly connected with elliptic functions: and to exhibit the connexion of the formulæ with the ordinary formulæ for elliptic integrals of the second and the third kinds respectively.

139. We have, in general,

$$
\{012\},=\widetilde{012} \frac{\pi}{012},=\left\{\begin{array}{l}
\left(1+k^{2}\right) x_{1} x_{2}-\left(x_{1}+x_{2}\right)+y_{1} y_{2} \\
+x\left[-1+\left(1+k^{2}\right)\left(x_{1}+x_{2}\right)\right. \\
+y\left(y_{1}+y_{2}\right)
\end{array}\right\} \div\left|\begin{array}{lll}
x, & y, & 1 \\
x_{1}, & y_{1}, & 1 \\
x_{2}, & y_{2}, & 1
\end{array}\right| .
$$


Taking here $2,=\theta$, for the point, coordinates $x_{2}, y_{2}=0$, 0 , we have

$$
\{01 \theta\}=\frac{-x_{1}-x+\left(1+k^{2}\right) x x_{1}+y y_{1}}{x y_{1}-x_{1} y}
$$

and if, retaining 1 to denote the point coordinates $\left(x_{1}, y_{1}\right)$ belonging to the argument $u_{1}$, we write $1^{\prime}$ for the point belonging to the argument $u+i K^{\prime}$, then the coordinates of $1^{\prime}$ are $\frac{1}{k^{2} x_{1}}, \frac{-y_{1}}{k^{2} x_{1}^{2}}$, and the formula becomes

$$
\left\{01^{\prime} \theta\right\}=\frac{x_{1}+k^{2} x x_{1}^{2}-\left(1+k^{2}\right) x x_{1}}{x y_{1}+x_{1} y} ;
$$

the numerator hereof multiplied by $x$ is $=y\left(x y_{1}+x_{1} y\right)-k^{2} x^{2} x_{1}\left(x-x_{1}\right)$, and we thence have

$$
\left\{01^{\prime} \theta\right\}=\frac{y}{x}-\frac{k^{2} x x_{1}\left(x-x_{1}\right)}{x y_{1}-x_{1} y},
$$

which, substituting for $x, y, x_{1}, y_{1}$ their values in terms of $u, u_{1}$, is

$$
=\frac{\operatorname{cn} u \operatorname{dn} u}{\operatorname{sn} u}-k^{2} \operatorname{sn} u \operatorname{sn} u_{1} \operatorname{sn}\left(u-u_{1}\right) \text {. }
$$

Operating on each side with $\frac{d}{d u_{1}},=\partial_{1}$, we obtain

$$
\partial_{1}\left\{01^{\prime} \theta\right\}=k^{2} \operatorname{sn}^{2} u_{1}-k^{2} \operatorname{sn}^{2}\left(u-u_{1}\right),
$$

the differentiation being, in fact, that which occurs in establishing the fundamental property of the elliptic integral of the second kind

$$
Z u=u\left(1-\frac{E}{K}\right)+k^{2} \int_{0} \operatorname{sn}^{2} u d u:
$$

viz. we have

$$
Z u-Z u_{1}-Z\left(u-u_{1}\right)=-k^{2} \operatorname{sn} u \operatorname{sn} u_{1} \operatorname{sn}\left(u-u_{1}\right) \text {, }
$$

and thence

$$
\partial_{1}\left[-k^{2} \operatorname{sn} u \operatorname{sn} u_{1} \operatorname{sn}\left(u-u_{1}\right)\right]=-Z^{\prime} u_{1}+Z^{\prime}\left(u-u_{1}\right),=k^{2} \operatorname{sn}^{2} u_{1}-k^{2} \operatorname{sn}^{2}\left(u-u_{1}\right) .
$$

Observe that, $1^{\prime}$ referring to $u_{1}+i k^{\prime}$, the subscript 1 might be written $1^{\prime}$.

The same result should of course be obtainable by the differentiation of the expression of $\left\{01^{\prime} \theta\right\}$ in terms of $x, y, x_{1}, y_{1}$. We have

$$
\partial_{1} x_{1}=2 y_{1}, \partial_{1} y_{1}=1-2\left(1+k^{2}\right) x_{1}+3 k^{2} x_{1}^{2},=\Omega_{1}
$$

and we thence obtain

$$
\partial_{1}\left\{01^{\prime} \theta\right\}=\frac{k^{2}}{\left(x y_{1}+x_{1} y\right)^{2}}\left[-2\left(x y_{1}+x_{1} y\right)\left(x-2 x_{1}\right) x y_{1}+x x_{1}\left(\bar{x}-x_{1}\right)\left(x \Omega_{1}+2 y y_{1}\right)\right],
$$

where the term in [ ] is found to be $=x\left(x y_{1}+x_{1} y\right)^{2}-x x_{1}\left(x-x_{1}\right)^{2}$; whence the equation is

giving the foregoing result.

$$
\partial_{1}\left\{01^{\prime} \theta\right\}=k^{2} x-\frac{k^{2} x x_{1}\left(x-x_{1}\right)^{2}}{\left(x y_{1}+x_{1} y\right)^{2}},
$$


140. To introduce into the formulæ 1 instead of $1^{\prime}$, we have only to write $u_{1}-i K^{\prime}$ instead of $u_{1}$; putting also for shortness $s, c, d, s_{1}, c_{1}, d_{1}$ for the functions of $u$ and $u_{1}$ respectively, we thus obtain

$$
\{01 \theta\}=-\frac{c d}{s}+\frac{s}{s_{1} \operatorname{sn}\left(u-u_{1}\right)},
$$

where observe that, interchanging $u$ and $u_{1}$, we have

$$
\{10 \theta\}=-\frac{c_{1} d_{1}}{s_{1}}-\frac{s_{1}}{s \operatorname{sn}\left(u-u_{1}\right)},
$$

that is, $\{10 \theta\}=-\{01 \theta\}$, as it should be. The formulæ may be written

and

$$
\{01 \theta\},=-\{10 \theta\},=\frac{s^{3} c_{1} d_{1}+s_{1}^{3} c d}{s c_{1} d_{1}+s_{1} c d} \frac{1}{s s_{1} \operatorname{sn}\left(u-u_{1}\right)}:
$$

$$
\partial_{1}\{01 \theta\}=\frac{1}{s_{1}^{2}}-\frac{1}{\operatorname{sn}^{2}\left(u-u_{1}\right)}
$$

whence

$$
\partial_{0}\{01 \theta\}=-\frac{1}{s^{2}}+\frac{1}{\operatorname{sn}^{2}\left(u-u_{1}\right)}
$$

we have, moreover,

$$
\{012\}=\{12 \theta\}+\frac{s}{s_{1} \operatorname{sn}\left(u-u_{1}\right)}-\frac{s}{s_{2} \operatorname{sn}\left(u-u_{1}\right)}
$$

and

$$
\partial_{0}\{012\}=\frac{1}{\operatorname{sn}^{2}\left(u-u_{1}\right)}-\frac{1}{\operatorname{sn}^{2}\left(u-u_{2}\right)},
$$

which last equation gives $\left(\partial_{0}+\partial_{1}+\partial_{2}\right)\{012\}=0$, as it should do.

141. Supposing that the differential $d \Pi_{12}$ is defined by the equation

we have

$$
d \Pi_{12}=d u\{012\}+d u\left[\int_{2}^{1} d u \partial_{3}\{036\}-\{123\}\right],
$$

$$
\int_{5}^{4} d \Pi_{12}=\int_{5}^{4} d u\{012\}+\int_{5}^{4} d u\left[\int_{2}^{1} d u \partial_{3}\{036\}-\{123\}\right]
$$

and thence

$$
\begin{aligned}
\partial_{1} \partial_{4} \int_{5}^{4} d \Pi_{12} & =\partial_{1}\{124\}+\partial_{3}\{136\}-\partial_{1}\{123\} \\
& =\partial_{1}\{134\}-\partial_{3}\{316\} \\
& =\left[\frac{1}{\operatorname{sn}^{2}\left(u_{1}-u_{8}\right)}-\frac{1}{\operatorname{sn}^{2}\left(u_{1}-u_{4}\right)}\right]-\left[\frac{1}{\operatorname{sn}^{2}\left(u_{3}-u_{1}\right)}-\frac{1}{\operatorname{sn}^{2}\left(u_{3}-u_{6}\right)}\right], \\
& =\frac{1}{\operatorname{sn}^{2}\left(u_{3}-u_{6}\right)}-\frac{1}{\operatorname{sn}^{2}\left(u_{1}-u_{4}\right)},
\end{aligned}
$$

C. XII. 
or establishing between the constants $u_{3}, u_{6}$ the relation $\operatorname{sn}^{2}\left(u_{3}-u_{6}\right)=\frac{K}{K-E}$, this becomes

$$
=1-\frac{E}{K}-k^{2} \mathrm{sn}^{2}\left(u_{4}-u_{1}+i K^{\prime}\right)
$$

which is

$$
=\partial_{2} \partial_{4} \log \frac{\Theta\left(u_{4}-u_{2}+i K^{\prime}\right) \Theta\left(u_{5}-u_{1}+i K^{\prime}\right)}{\Theta\left(u_{4}-u_{1}+i K^{\prime}\right) \Theta\left(u_{5}-u_{2}+i K^{\prime}\right)}
$$

where $\Theta$ is Jacobi's theta-function, see my Elliptic Functions, p. 144. The expression is, in fact, $=-\partial_{1} \partial_{4} \log \theta\left(u_{4}-u_{1}+i K^{\prime}\right),=\phi\left(u_{4}-u_{1}+i K^{\prime}\right)$, if for a moment $\phi v=\partial_{v}{ }^{2} \log \Theta v$. But we have

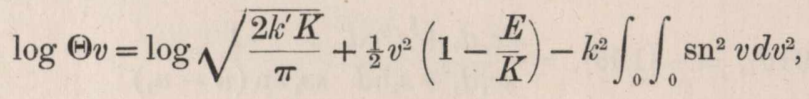

that is, $\phi v=1-\frac{E}{K}-k^{2} \operatorname{sn}^{2} v$, and consequently we have $\phi\left(u_{4}-u_{1}+i K^{\prime}\right)$

$$
=1-\frac{E}{K}-k^{2} \operatorname{sn}^{2}\left(u_{4}-u_{1}+i K^{\prime}\right) .
$$

142. In connexion with the same curve $y^{2}=x(1-x)\left(1-k^{2} x\right)$, we may establish the identity

$$
\frac{d}{d u_{1}} \frac{y_{1}}{x_{1}-x}+\frac{d}{d u} \frac{y}{x_{1}-x}=k^{2}\left(x_{1}-x\right),
$$

where as before $x, y=s^{2}, s c d$, and $x_{1}, y_{1}=s_{1}^{2}, s_{1} c_{1} d_{1}$. We have

$$
\begin{aligned}
\left(x_{1}-x\right) \frac{d y_{1}}{d u_{1}}-y_{1} \frac{d x_{1}}{d u_{1}} & =\left(s_{1}{ }^{2}-s^{2}\right)\left\{1-2\left(1+k^{2}\right) s_{1}{ }^{2}+3 k^{2} s_{1}{ }^{4}\right\}-2 s_{1}{ }^{2}\left(1-s_{1}{ }^{2}\right)\left(1-k^{2} s_{1}{ }^{2}\right) \\
& =-s^{2}-s_{1}{ }^{2}+2\left(1+k^{2}\right) s^{2} s_{1}{ }^{2}-3 k^{2} s^{2} s_{1}{ }^{4}+k^{2} s_{1}{ }^{6}
\end{aligned}
$$

and similarly

$$
\left(x-x_{1}\right) \frac{d y}{d u}-y \frac{d x}{d u}=-s^{2}-s_{1}^{2}+2\left(1+k^{2}\right) s^{2} s_{1}{ }^{2}-3 k^{2} s^{4} s_{1}{ }^{2}+k^{2} s^{6} .
$$

The difference of the two functions on the right-hand side is $=k^{2}\left(s_{1}{ }^{2}-s^{2}\right)^{3}$; which is $=k^{2}\left(x_{1}-x\right)^{3}$, and this divided by $\left(x_{1}-x\right)^{2}$ is $=k^{2}\left(x_{1}-x\right)$; the identity is thus verified.

Fixed Curve the Quartic $y^{2}=\left(1-x^{2}\right)\left(1-k^{2} x^{2}\right)$. Art. Nos. 143 to 145 .

143. This is a curve having a tacnode at infinity on the line $x=0$, as may be seen by writing the equation in the homogeneous form $y^{2} z^{2}=\left(z^{2}-x^{2}\right)\left(z^{2}-k^{2} x^{2}\right)$; we have as it were two branches having the line infinity for a common tangent at the point in question. The equation is satisfied by $x=\operatorname{sn} u, y=\mathrm{cn} u \mathrm{dn} u$, values which are unaltered by the change of $u$ into $u+4 m K+2 m^{\prime} i K^{\prime}, m$ and $m^{\prime}$ any positive or negative integers; in regard to this curve, the sign $\equiv$ is to be understood accordingly. I consider with 
reference to this curve only the affected theorem, in the particular form in which it most readily connects itself with the ordinary theory of the integral of the third kind.

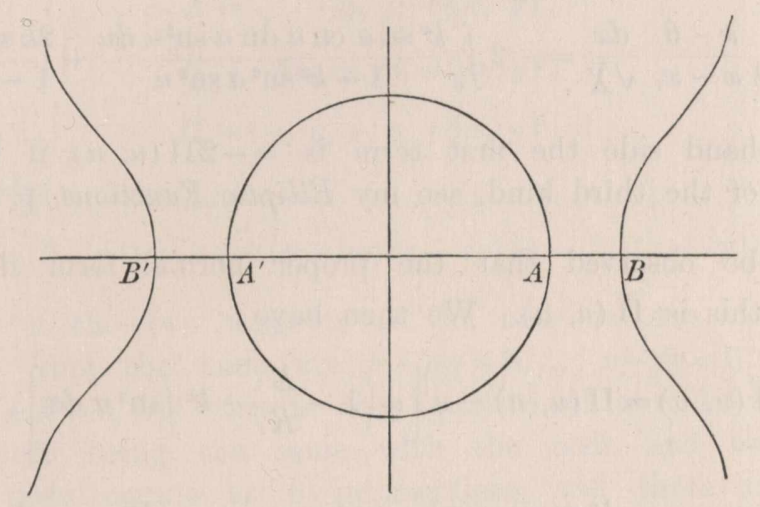

144. I consider the differential $\frac{(x, y, z)^{2}{ }_{21} d \omega}{012}$ in the particular case where the line 012 is a line parallel to the axis of $y$ : taking its equation to be $x-x_{1}=0$, and putting for shortness $X=\sqrt{1-x^{2} \cdot 1-k^{2} x^{2}}, X_{1}=\sqrt{1-x_{1}{ }^{2} \cdot 1-k^{2} x_{1}^{2}}$, the parametric points are taken to be $\left(x_{1}, \sqrt{X_{1}}\right),\left(x_{1},-\sqrt{X_{1}}\right)$, and the residues are the intersections with the two branches at the tacnode. The conic $(x, y, z)^{2}{ }_{12}=0$ is to satisfy the conditions of passing through the two nodes of the tacnode, and through the two residues, that is, again through the tacnode twice-in all, four conditions; and we have thus the form $z(x-\theta z)=0$, containing the arbitrary constant $\theta$. The major function itself is then easily determined, and putting again $z=1$ we arrive at the form

$$
\frac{\sqrt{X_{1}}}{x_{1}-\theta} \frac{x-\theta}{x-x_{1}} \frac{d x}{\sqrt{X}}
$$

If the limits are taken to be two points on a line parallel to the axis of $x$, or what is the same thing, if the limits in regard to $x$ are $x,-x$, we have the integral

$$
\begin{aligned}
\int_{-x}^{x} \frac{\sqrt{X_{1}}}{x_{1}-\theta} \frac{x-\theta}{x-x_{1}} \frac{d x}{\sqrt{X}}, & =\int_{0}^{x} \frac{\sqrt{X_{1}}}{x_{1}-\theta}\left(\frac{x-\theta}{x-x_{1}}+\frac{x+\theta}{x+x_{1}}\right) \frac{d x}{\sqrt{X}}, \\
& =2 \int_{0}^{x} \frac{\sqrt{X_{1}}}{x_{1}-\theta} \cdot \frac{x^{2}-x_{1} \theta}{x^{2}-x_{1}{ }^{2}} \frac{d x}{\sqrt{X}} .
\end{aligned}
$$

We have

$$
\frac{1}{x_{1}}\left(\frac{x^{2}}{x_{1}^{2}-x^{2}}-\frac{\theta}{x_{1}-\theta}\right)=\frac{x^{2}-x_{1} \theta}{x_{1}^{2}-x^{2} \cdot x_{1}-\theta},
$$

and the integral thus becomes

$$
=-2 \int_{0}^{x} \frac{\sqrt{X_{1}} x^{2} d x}{x_{1}\left(x_{1}^{2}-x^{2}\right) \sqrt{X}}+2 \frac{\theta \sqrt{X_{1}}}{x_{1}\left(x_{1}-\theta\right)} \int_{0}^{x} \frac{d x}{\sqrt{X}} .
$$


Taking here $x=\operatorname{sn} u, x_{1}=\operatorname{sn}\left(a+i K^{\prime}\right),=\frac{1}{k \operatorname{sn} a}$, we have $d x=\operatorname{cn} u \operatorname{dn} u d u=\sqrt{X} d u$, and the result is

$$
\int_{-x}^{x} \frac{\sqrt{X_{1}}}{x_{1}-\theta} \frac{x-\theta}{x-x_{1}} \frac{d x}{\sqrt{X}}=-2 \int_{0}^{k^{2} \operatorname{sn} a \operatorname{cn} a \operatorname{dn} a \operatorname{sn}^{2} u d u} \frac{2 k \operatorname{sn} a \operatorname{cn} a}{1-k^{2} \operatorname{sn}^{2} a \operatorname{sn}^{2} u}+\frac{1-k \theta \operatorname{sn} a}{1-k}
$$

where on the right-hand side the first term is $=-2 \Pi(u, a)$, if $\Pi(u, a)$ be Jacobi's form of the integral of the third kind, see my Elliptic Functions, p. 143.

145. It is to be observed that the proper normal form is not $\Pi(u, a)$, but $\Pi(u, a)-u Z a$; say this is $\bar{\Pi}(u, a)$. We then have

and thence

$$
\bar{\Pi}(u, a)=\Pi(u, a)-u\left[a\left(1-\frac{E}{K}\right)-k^{2} \int \operatorname{sn}^{2} a d a\right],
$$

$$
\begin{gathered}
\partial_{u} \bar{\Pi}(u, a)=\frac{k^{2} \operatorname{sn} a \operatorname{cn} a \operatorname{dn} a \operatorname{sn}^{2} u}{1-k^{2} \operatorname{sn}^{2} a \operatorname{sn}^{2} u}-a\left(1-\frac{E}{K}\right)+k^{2} \int \operatorname{sn}^{2} a d a \\
\partial_{a} \partial_{u} \bar{\Pi}(u, a)=k^{2} \operatorname{sn}^{2} u \partial_{a} \frac{\operatorname{sn} a \operatorname{cn} a \operatorname{dn} a}{1-k^{2} \operatorname{sn}^{2} a \operatorname{sn}^{2} u}-\left(1-\frac{E}{K}\right)+k^{2} \operatorname{sn}^{2} a
\end{gathered}
$$

or, if for shortness we write $\operatorname{sn} u, \operatorname{sn} a=s, \sigma$, this is

$$
\partial_{a} \partial_{u} \bar{\Pi}(u, a)=\frac{k^{2} s^{2}\left[1-2\left(1+k^{2}\right) \sigma^{2}+3 k^{2} \sigma^{4}\right]+k^{4} s^{4} \sigma^{2}}{\left(1-k^{2} s^{2} \sigma^{2}\right)^{2}}-\left(1-\frac{E}{K}\right)+k^{2} \sigma^{2},
$$

which is

$$
=\frac{k^{2}\left\{\left(s^{2}+\sigma^{2}\right)\left(1+k^{2} s^{2} \sigma^{2}\right)-2\left(1+k^{2}\right) s^{2} \sigma^{2}\right\}}{\left(1-k^{2} s^{2} \sigma^{2}\right)^{2}}-\left(1-\frac{E}{K}\right)
$$

or, this being symmetrical in regard to $s, \sigma$, we have

$$
\partial_{a} \partial_{u} \bar{\Pi}(u, a)=\partial_{a} \partial_{u} \bar{\Pi}(a, u),
$$

and thence by integration, and a proper determination of the constants,

$$
\bar{\Pi}(u, a)=\bar{\Pi}(a, u) .
$$

\section{Chapter VI. The Nodal Quartic.}

Nodal Quartic; the General and Fleflecnodal Forms. Art. Nos. 146 to 148.

146. For a cubic, or other curve of deficiency 1, we are concerned with single points on the curve, and corresponding thereto with functions of a single argument (elliptic functions): but for a curve of deficiency 2, we have to consider pairs of points on the curve, and functions of two arguments: there is thus a marked change in the form of the results. 
The most simple curve of deficiency 2 is the nodal quartic, $n=4, p=2$. Using homogeneous coordinates, the general form is $A z^{2}+2 B z+C=0$, where

$$
\begin{aligned}
& A=(i, j, k \gamma x, y)^{2}, \\
& B=(l, m, n, o \gamma x, y)^{3}, \\
& C=(p, q, r, s, t \gamma x, y)^{4},
\end{aligned}
$$

and where we write also

$$
B^{2}-A C=\left(l^{2}-i p\right)(x-a y)(x-b y)(x-c y)(x-d y)(x-e y)(x-f y) .
$$

Clearly the equation of the two tangents at the node is $A=0$; and the equations of the six tangents from the node are $x-a y=0, \ldots, x-f y=0$ : at the points of contact we have $A z+B=0$, viz. this is the equation of a nodal cubic, the node and the two tangents there being the same with the node and two tangents of the quartic. Hence the node counts as 6 intersections, and there are besides 6 intersections which are the points of contact of the 6 tangents respectively: say these are the points $a, b, c, d, e, f$ : the coordinates of the point $a$ are given by the equations

$$
x: y: z=a: 1:-\frac{B_{a}}{A_{a}}\left(=-\frac{C_{a}}{B_{a}}\right),
$$

where $A_{a}, B_{a}, C_{a}$ are what $A, B, C$ become on writing therein $a, 1$ for $x, y$ : and similarly for the other points.

147. An important special case occurs when $B=0$; say we have here

$$
\begin{aligned}
& A=i(x-e y)(x-f y) \\
& B=0 \\
& C=p(x-a y)(x-b y)(x-c y)(x-d y)
\end{aligned}
$$

or, omitting the factors $i$ and $p$,

$$
(x-e y)(x-f y) z^{2}=(x-a y)(x-b y)(x-c y)(x-d y) .
$$

The origin is here a fleflecnode; the tangents $x-e y=0, x-f y=0$ count as two of the six tangents from the node, and there remain the four tangents

$$
x-c y=0, \quad x-d y=0, \quad x-a y=0, \quad x-b y=0 ;
$$

the four points of contact are the intersections of the curve with the line $z=0$.

148. The general nodal form depends on 11 constants, but by writing $\alpha x+\beta y$, $\gamma x+\delta y, \epsilon z$ in place of $x, y, z$, we introduce 5 apoclastic constants, and so reduce the number to $11+1-5,=7$. Similarly the fleflecnodal form depends on 7 constants, but we reduce the number in like manner to $7+1-5,=3$ : the final form might here be taken to be

$$
z^{2} x y=(x-y)(x-b y)(x-c y)(x-d y)
$$


but it is more convenient to retain the original form

$$
z^{2}(x-e y)(x-f y)=(x-a y)(x-b y)(x-c y)(x-d y),
$$

bearing in mind that this is reducible to the form just referred to, and thus depends virtually upon only 3 constants.

It is a general property that a curve of deficiency $p$ greater than 1 can be by a rational transformation reduced to a curve of that deficiency depending upon $3 p-3$ parameters: in particular, if $p=2$, then the form depending upon 3 parameters may be taken to be the fleflecnodal quartic as above: and I proceed to show how the general nodal quartic can, in fact, be reduced to this fleflecnodal form.

\section{Reduction to the Fleflecnodal Form. Art. Nos. 149 to 152.}

149. Consider the general nodal quartic $A z^{2}+2 B z+C=0$ : take $\zeta=0$ for the equation of the line joining the points of contact of the tangents $x-e y=0, x-f y=0$; and then writing $x=\xi, y=\eta$, let the curve be transformed in the first instance from $x, y, z$ to the new coordinates $\xi, \eta, \zeta$.

Writing $A_{e}$ for the value $\left(i, j, k \gamma_{\ell}, 1\right)^{2}$, which $A$ assumes on putting therein $(e, 1)$ for $(x, y)$ respectively, and similarly $A_{f}, B_{e}, B_{f}$ for the other like values, we may take

$$
\begin{aligned}
& A_{e} A_{f}(e-f) \zeta=\left|\begin{array}{ccc}
x & y, & z \\
e A_{e}, & A_{e}, & -B_{e} \\
f A_{f}, & A_{f}, & -B_{f}
\end{array}\right|, \\
& =-x\left(A_{e} B_{f}-A_{f} B_{e}\right)+y\left(e A_{e} B_{f}-f B_{e} A_{f}\right)+z(e-f) A_{e} A_{f},
\end{aligned}
$$

say this equation is $\zeta=-\lambda x-\mu y+z$, the values of $\lambda$, $\mu$ being

and therefore

$$
\lambda=\frac{A_{e} B_{f}-A_{f} B_{e}}{(e-f) A_{e} A_{f}}, \quad \mu=\frac{-e A_{e} B_{f}+f B_{e} A_{f}}{(e-f) A_{e} A_{f}},
$$

$$
\lambda e+\mu=-\frac{B_{e}}{A_{e}}, \quad \lambda f+\mu=-\frac{B_{f}}{A_{f}} .
$$

150. From the values $\zeta, \xi, \eta=-\lambda x-\mu y+z, x, y$, we obtain $z, x, y=\zeta+\lambda \xi+\mu \eta, \xi, \eta$; and the transformed equation is

where

$$
A^{\prime}(\zeta+\lambda \xi+\mu \eta)^{2}+2 B^{\prime}(\zeta+\lambda \xi+\mu \eta)+C^{\prime}=0
$$

$$
\begin{aligned}
& A^{\prime}=(i, j, k \gamma \xi, \eta)^{2}, \\
& B^{\prime}=\left(l, m, n, o_{\gamma \xi} \xi \eta\right)^{3}, \\
& C^{\prime}=(p, q, r, s, t \gamma \xi, \eta)^{4},
\end{aligned}
$$

say this equation is $\mathfrak{2} \zeta^{2}+2 \mathfrak{B} \zeta+\mathfrak{S}=0$, where

$$
\begin{aligned}
& \mathfrak{A}=A^{\prime}, \\
& \mathfrak{B}=A^{\prime}(\lambda \xi+\mu \eta)+B^{\prime}, \\
& \mathfrak{C}=A^{\prime}(\lambda \xi+\mu \eta)^{2}+2 B^{\prime}(\lambda \xi+\mu \eta)+C^{\prime},
\end{aligned}
$$


and thence

$$
\mathfrak{B}^{2}-\Re\left(\zeta=B^{\prime 2}-A^{\prime} C^{\prime},=\left(l^{2}-i p\right)(\xi-a \eta)(\xi-b \eta)(\xi-c \eta)(\xi-d \eta)(\xi-e \eta)(\xi-f \eta) .\right.
$$

We have here $\mathfrak{B},=A^{\prime}(\lambda \xi+\mu \eta)+B^{\prime}$, a cubic function $(\xi, \eta)^{3}$ containing the factors $\xi-e \eta$ and $\xi-f \eta$ : in fact, writing $\xi, \eta=e, 1$, it becomes $A_{e}(\lambda e+\mu)+B_{e}$, which is $=0$; and similarly writing $\xi, \eta=f, 1$, it becomes $A_{f}(\lambda f+\mu)+B_{f}$, which is $=0$. Calling the other factor $L \xi+M \eta$, we have thus

and thence

$$
\mathfrak{B}=(\xi-e \eta)(\xi-f \eta)(L \xi+M \eta)
$$

$$
\begin{aligned}
\mathfrak{A}(\zeta & =(\xi-e \eta)^{2}(\xi-f \eta)^{2}(L \xi+M \eta)^{2}-\left(l^{2}-i p\right)(\xi-a \eta)(\xi-b \eta)(\xi-c \eta)(\xi-d \eta)(\xi-e \eta)(\xi-f \eta), \\
& =(\xi-e \eta)(\xi-f \eta)\left[(\xi-e \eta)(\xi-f \eta)(L \xi+M \eta)^{2}-\left(l^{2}-i p\right)(\xi-c \eta)(\xi-d \eta)(\xi-e \eta)(\xi-f \eta)\right] .
\end{aligned}
$$

Hence $(5$ contains the factor $(\xi-e \eta)(\xi-f \eta)$, say we have

$$
(\widetilde{\xi}=\theta(\xi-e \eta)(\xi-f \eta)(\xi-\epsilon \eta)(\xi-\phi \eta) .
$$

151. In the equation $\mathfrak{A} \zeta^{2}+2 \mathfrak{B} \zeta+(\overleftarrow{\zeta}=0$ of the quartic curve, writing $\zeta=0$, we find $\delta=0$, that is, $(\xi-e \eta)(\xi-f \eta)(\xi-\epsilon \eta)(\xi-\phi \eta)=0$ : but $\zeta=0$ is the equation of the line joining the points of contact of the tangents $\xi-e \eta=0, \xi-f \eta=0$; hence $\xi-\epsilon \eta=0, \xi-\phi \eta=0$ are the lines drawn from the node to the two points $\epsilon, \phi$ which are the residues of thése two points of contact. We now have

$$
\theta \mathfrak{A}(\xi-\epsilon \eta)(\xi-\phi \eta)=(\xi-e \eta)(\xi-f \eta)(L \xi+M \eta)^{2}-\left(l^{2}-i p\right)(\xi-a \eta)(\xi-b \eta)(\xi-c \eta)(\xi-d \eta),
$$

and thence

$$
\begin{aligned}
& 0=(\epsilon-e)(\epsilon-f)(L \epsilon+M)^{2}-\left(l^{2}-i p\right)(\epsilon-a)(\epsilon-b)(\epsilon-c)(\epsilon-d) \\
& 0=(\phi-e)(\phi-f)(L \phi+M)^{2}-\left(l^{2}-i p\right)(\phi-a)(\phi-b)(\phi-c)(\phi-d)
\end{aligned}
$$

which equations determine $L$ and $M$; and then with these values of $L, M$, and for $\mathfrak{A}$ substituting its value $(i, j, k \gamma \xi, \eta)^{2}$, the equation must become an identity.

We have in what precedes, by the transformation $z=\zeta+\lambda \xi+\mu \eta, x=\xi, y=\eta$, passed from the form $A z^{2}+2 B z+C=0$ to the form

where

$$
\mathfrak{A} \zeta^{2}+2 \mathfrak{B} \zeta+\mathfrak{C}=0
$$

$$
\begin{aligned}
& \mathfrak{A}=(i, j, k)(\xi, \eta)^{2}, \\
& \mathfrak{B}=(\xi-e \eta)(\xi-f \eta)(L \xi+M \eta), \\
& \mathfrak{E}=\theta(\xi-e \eta)(\xi-f \eta)(\xi-\epsilon \eta)(\xi-\phi \eta),
\end{aligned}
$$

viz. $\mathfrak{B}$ and $(5$ have here the common factor $(\xi-e \eta)(\xi-f \eta)$.

152. Assume now

$$
\xi, \eta, \zeta=X, Y, \theta \frac{(X-\epsilon Y)(X-\phi Y)}{Z-L X-M Y},
$$

and therefore conversely

$$
X, Y, Z=\xi, \eta, L \xi+M \eta+\theta \frac{(\xi-\epsilon \eta)(\xi-\phi \eta)}{\zeta}
$$


then in the new coordinates $(X, Y, Z)$ we have the equation

$$
\begin{gathered}
(i, j, k)(X, Y)^{2} \theta^{2}\left\{\frac{(X-\epsilon Y)(X-\phi Y)}{Z-L X-M Y}\right\}^{2} \\
+2(X-e Y)(X-f Y)(L X+M Y) \theta \frac{(X-\epsilon Y)(X-\phi Y)}{Z-L X-M Y} \\
+\theta(X-e Y)(X-f Y)(X-\epsilon Y)(X-\phi Y)=0,
\end{gathered}
$$

that is,

$$
\begin{aligned}
& (i, j, k \gamma X, Y)^{2} \theta(X-\epsilon Y)(X-\phi Y) \\
+ & 2(X-e Y)(X-f Y)(L X+M Y)(Z-L X-M Y) \\
+ & (X-e Y)(X-f Y)(Z-L X-M Y)^{2}=0
\end{aligned}
$$

where the second and third lines together are

$$
=(X-e Y)(X-f Y)\left\{Z^{2}-(L X+M Y)^{2}\right\},
$$

and the equation thus is

$$
\begin{aligned}
(X-e Y)(X-f Y) Z^{2}+\left\{\theta(i, j, k \gamma X, Y)^{2}(X-\epsilon Y)(X-\phi Y)\right. \\
\left.-(X-e Y)(X-f Y)(L X+M Y)^{2}\right\}=0 .
\end{aligned}
$$

But the term in \{\} is identically

$$
=-\left(l^{2}-i p\right)(X-a Y)(X-b Y)(X-c Y)(X-d Y),
$$

and the equation thus becomes

$$
(X-e Y)(X-f Y) Z^{2}-\left(l^{2}-i p\right)(X-a Y)(X-b Y)(X-c Y)(X-d Y)=0 ;
$$

viz. the original equation $A z^{2}+2 B z+C=0$ of the general nodal quartic is, by the equations

$$
x, y, z=X, Y, \lambda X+\mu Y+\theta \frac{(X-\epsilon Y)(X-\phi Y)}{Z-L X-M Y},
$$

or conversely

$$
X, Y, Z=x, y, L x+M y+\frac{\theta(x-\epsilon y)(x-\phi y)}{z-\lambda x-\mu y}
$$

transformed into the fleflecnodal form as above.

It originally appeared to me that the fleflecnodal form was more easily dealt with than the general form; and I effected the transformation for this reason: there is, however, the disadvantage that the six points $a, b, c, d, e, f$ enter into the equation unsymmetrically; and I afterwards found that the general form could be dealt with nearly as easily, and in what follows I use therefore the general form. The transformation is given as interesting for its own sake, and as an illustration of the theorem in regard to the number of constants in a curve of deficiency $p$. 
Application of Abel's Theorem. Art. Nos. 153 to 157.

153. Taking the fixed curve to be $f,=\frac{1}{2}\left(A z^{2}+2 B z+C\right)$, $=0$, we have

$$
\frac{d f}{d z}=A z+B=\sqrt{(x, y)^{6}}
$$

if for shortness we write

$$
(x, y)^{6}=B^{2}-A C,=\left(l^{2}-i p\right)(x-a y)(x-b y)(x-c y)(x-d y)(x-e y)(x-f y),
$$

and we thence have

$$
d \omega=\frac{x d y-y d x}{\sqrt{(x, y)^{6}}}
$$

The minor curve $(x, y, z)^{n-3}=0$ is an arbitrary line passing through the node, that is, the point $x=0, y=0$; and the pure theorem thus gives the two relations $\Sigma x d \omega=0, \Sigma y d \omega=0$; where the summation extends to the intersections of the fixed curve $A z^{2}+2 B z+C=0$ with the variable curve $\phi$.

The variable curve is taken to be a cubic $A z+B=\left(\alpha, \beta, \gamma, \delta \gamma(x, y)^{3}\right.$, or say $A z+B=\Omega$, where $\Omega$ is a given cubic function $(x, y)^{3}$ : viz. this is a nodal cubic, the node and the two tangents there being the same with the node and the two tangents of the quartic: hence it meets the quartic in the node counting 6 times, and in 6 other points, say these are the points $1,2,3,4,5,6$ : hence the differential relations are

$$
\begin{aligned}
& x_{1} d \omega_{1}+x_{2} d \omega_{2}+x_{3} d \omega_{3}+x_{4} d \omega_{4}+x_{5} d \omega_{5}+x_{6} d \omega_{6}=0 \\
& y_{1} d \omega_{1}+y_{2} d \omega_{2}+y_{3} d \omega_{3}+y_{4} d \omega_{4}+y_{5} d \omega_{5}+y_{6} d \omega_{6}=0 .
\end{aligned}
$$

154. Observe that the intersections of the cubic with the fixed curve are given by the equation $\Omega^{2}=B^{2}-A C$, or say $\Omega^{2}=(x, y)^{6}$, an equation which determines the ratio $x: y$ for the six points respectively; and the ratio $z: x$ is then determined rationally by the original equation $A z+B=\Omega$. Instead of regarding $\Omega$ as a given function, we may, if we please, take $1,2,3,4$ given points on the quartic: we then have four equations for the determination of the coefficients $(\alpha, \beta, \gamma, \delta)$ of the function $\Omega$; viz. these equations may be taken to be

$$
\begin{aligned}
& (\alpha, \beta, \gamma, \delta)\left(x_{1}, y_{1}\right)^{3}=\sqrt{\left(x_{1}, y_{1}\right)^{6}}, \\
& \text { ) }\left(x_{2}, y_{2}\right)^{3}=\sqrt{\left(x_{2}, y_{2}\right)^{6}} \text {, } \\
& \text { ) }\left(x_{3}, y_{3}\right)^{3}=\sqrt{\left(x_{3}, y_{3}\right)^{6}}, \\
& \text { ) }\left(x_{4}, y_{4}\right)^{3}=\sqrt{\left(x_{4}, y_{4}\right)^{6}} \text {. }
\end{aligned}
$$

$\Omega$ is hereby completely determined: and this being so, the remaining intersections 5 and 6 are also completely determined: there are thus between the six points 2 integral relations, which agrees with the number, $=2$, of the differential relations obtained above. 
155. If we now assume

or say

$$
\begin{array}{lll}
d u=x_{1} d \omega_{1}+x_{2} d \omega_{2}, & d u^{\prime}=x_{3} d \omega_{3}+x_{4} d \omega_{4}, & d u^{\prime \prime}=x_{5} d \omega_{5}+x_{6} d \omega_{6}, \\
d v=y_{1} d \omega_{1}+y_{2} d \omega_{2}, & d v^{\prime}=y_{3} d \omega_{3}+y_{4} d \omega_{4}, & d v^{\prime \prime}=y_{5} d \omega_{5}+y_{6} d \omega_{6},
\end{array}
$$

that is,

$$
\begin{aligned}
& u, u^{\prime}, u^{\prime \prime}=\int_{\alpha \beta}^{12}, \int_{\alpha \beta}^{34}, \int_{\alpha \beta}^{56} \frac{x(x d y-y d x)}{\sqrt{(x, y)^{6}}}, \\
& v, v^{\prime}, v^{\prime \prime}=\int_{\alpha \beta}^{12}, \int_{\alpha \beta}^{34}, \int_{\alpha \beta}^{56} \frac{y(x d y-y d x)}{\sqrt{(x, y)^{6}}} ;
\end{aligned}
$$

$$
u=\left(\int_{a}^{1}+\int_{\beta}^{2}\right) \frac{x(x d y-y d x)}{\sqrt{(x, y)^{6}}}, \quad v=\left(\int_{a}^{1}+\int_{\beta}^{2}\right) \frac{y(x d y-y d x)}{\sqrt{(x, y)^{6}}},
$$

where $\alpha, \beta$ are points assumed at pleasure on the quartic: and similarly for $u^{\prime}, v^{\prime}: u^{\prime \prime}, v^{\prime \prime}$ : then $u, v$ are hereby determined as functions of the points 1,2: and we may conversely regard the points 1,2 as determined in terms of the two arguments $u, v$. We might, selecting any two symmetrical functions of the degree zero, for instance, $\frac{x_{1}}{y_{1}}+\frac{x_{2}}{y_{2}}, \frac{x_{1} x_{2}}{y_{1} y_{2}}$, represent them as functions $\phi(u, v), \psi(u, v)$; and then $\frac{x_{1}}{y_{1}}$ and $\frac{x_{2}}{y_{2}}$ will be functions of $\phi(u, v), \psi(u, v)$. But instead of this selection, it is proper to consider the ratios of six functions depending on the points $a, b, c, d, e, f$ respectively: viz. we assume

$$
\begin{aligned}
& \left.\sqrt{\left(x_{1}-a y_{1}\right)\left(x_{2}-a y_{2}\right)}: \sqrt{\left(x_{1}-b y_{1}\right)\left(x_{2}-b y_{2}\right)}\right) \ldots: \sqrt{\left(x_{1}-f y_{1}\right)\left(x_{2}-f y_{2}\right)} \\
& =A(u, v) \quad: \quad B(u, v) \quad \therefore: \quad F(u, v) \text {, }
\end{aligned}
$$

and of course 3,4 will be in like manner determined by means of the corresponding functions of $u^{\prime}, v^{\prime}$, and 5,6 by means of the corresponding functions of $u^{\prime \prime}, v^{\prime \prime}$. The squared functions $A^{2}, B^{2}, C^{2}, D^{2}, E^{2}, F^{2}$ are proportional to given linear functions of $x_{1} x_{2}, x_{1} y_{2}+x_{2} y_{1}, y_{1} y_{2}$, and are thus connected by three independent linear relations.

156. The differential relations then become

$$
d u+d u^{\prime}+d u^{\prime \prime}=0, \quad d v+d v^{\prime}+d v^{\prime \prime}=0,
$$

and we have consequently

$$
u+u^{\prime}+u^{\prime \prime}=I, \quad v+v^{\prime}+v^{\prime \prime}=J,
$$

where $I, J$ are constants which are determinable as definite integrals by the consideration that, when the cubic is taken to be $A z+B=0$, the six points $1,2,3,4,5,6$ coincide with the points of contact $a, b, c, d, e, f$. I do not at present see my way to a proper development of this point of the theory: but in explanation of the nature of the result, I assume for the moment that by a proper determination of the inferior limits $\alpha, \beta$, or otherwise, we may take $I=0, J=0$. We then have $u^{\prime \prime}=-u-u^{\prime}, v^{\prime \prime}=-v-v^{\prime}$; and the integral equations, which determine the points 5,6 in terms of the points 1,2 and the points 3,4 , then in effect determine the functions $A, B$, \&c., of $-u-u^{\prime},-v-v^{\prime}$, or say those of $u+u^{\prime}, v+v^{\prime}$ in terms of the like functions of $(u, v)$ and of $\left(u^{\prime}, v^{\prime}\right)$ : viz. these equations give the addition-theory of the functions $A(u, v)$, \&c. 
157. We may, in the first instance, disregarding altogether the consideration of the arguments $u, v, \& c$., attend only to the algebraic functions such as $\sqrt{\left(x_{1}-a y_{1}\right)\left(x_{2}-\alpha y_{2}\right)}$, \&c., of the coordinates of the pairs of points 1,$2 ; 3,4$, and 5,6 ; and we can in regard to these develope a proper theory. This depends only on the equation $\Omega=\sqrt{(x, y)^{6}}$; it will be convenient to assume herein $y=1$, and slightly modifying the form, to write it

$$
(\alpha, \beta, \gamma, \delta)(x, 1)^{3}=\sqrt{a-x . b-x . c-x . d-x . e-x . f-x} ;
$$

and accordingly to consider the functions $\sqrt{a-x_{1} \cdot a-x_{2}}$, \&c. These are called the single-letter functions $A$, \&c., but there are certain double-letter functions $A B$, \&c., which have also to be considered; and I will, in the first instance, show how these present themselves in connexion with the cubic curve.

Origin of the Double-Letter Functions. Art. Nos. 158 and 159.

158. The cubic curve $A z+B=\Omega$ may be taken to be a curve through two of the points of contact, say the points $a, b$; these will then be two out of the six points, and taking the remaining four points to be the pairs 1,2 and 3, 4, we have single-letter functions of 3,4 presenting themselves as double-letter functions of $1,2$. In fact, the equation of the curve is

$$
A z+B=\lambda(x-a y)(x-b y)(x-k y) ;
$$

for the intersections with the quartic we have $\lambda^{2}(x-a y)^{2}(x-b y)^{2}(x-k y)^{2}=\Omega^{2}$, or throwing out the factor $(x-a y)(x-b y)$ and changing the constant $\lambda$, this is

$$
(x-a y)(x-b y)(x-k y)^{2}-\lambda(x-c y)(x-d y)(x-e y)(x-f y)=0 ;
$$

and the quartic function must be a multiple of

$$
\left(x y_{1}-x_{1} y\right)\left(x y_{2}-x_{2} y\right)\left(x y_{3}-x_{3} y\right)\left(x y_{4}-x_{4} y\right) .
$$

Putting each of the $y$ 's equal 1 , we have the identity

$$
(a-x)(b-x)(k-x)^{2}-\lambda(c-x)(d-x)(e-x)(f-x)=\mu\left(x_{1}-x\right)\left(x_{2}-x\right)\left(x_{3}-x\right)\left(x_{4}-x\right) ;
$$

and hence, introducing a notation which will be convenient, $a-x=\mathrm{a}, a-x_{1}=\mathrm{a}_{1}$, and so in other cases, we have by giving different values to $x$ the equations

$$
\begin{array}{lr}
\mathrm{a}_{1} \mathrm{~b}_{1} \mathrm{k}_{1}{ }^{2}=\lambda \mathrm{c}_{1} \mathrm{~d}_{1} \mathrm{e}_{1} \mathrm{f}_{1}, & (a-c)(b-c)(k-c)^{2}=\mu \mathrm{c}_{1} \mathrm{c}_{2} \mathrm{c}_{3} \mathrm{e}_{4}, \\
\mathrm{a}_{2} \mathrm{~b}_{2} \mathrm{k}_{2}{ }^{2}=\lambda \mathrm{c}_{2} \mathrm{~d}_{2} \mathrm{e}_{2} \mathrm{f}_{2}, & (a-d)(b-d)(k-d)^{2}=\mu \mathrm{d}_{1} \mathrm{~d}_{2} \mathrm{~d}_{3} \mathrm{~d}_{4}, \\
\mathrm{a}_{3} \mathrm{~b}_{3} \mathrm{k}_{3}{ }^{2}=\lambda \mathrm{c}_{3} \mathrm{~d}_{3} \mathrm{e}_{3} \mathrm{f}_{3}, & (a-e)(b-e)(k-e)^{2}=\mu \mathrm{e}_{1} \mathrm{e}_{2} \mathrm{e}_{3} \mathrm{e}_{4}, \\
\mathrm{a}_{4} \mathrm{~b}_{4} \mathrm{k}_{4}{ }^{2}=\lambda \mathrm{c}_{4} \mathrm{~d}_{4} \mathrm{e}_{4} \mathrm{f}_{4}, & (a-f)(b-f)(k-f)^{2}=\mu \mathrm{f}_{1} \mathrm{f}_{2} \mathrm{f}_{3} \mathrm{f}_{4}, \\
& -\lambda(c-a)(d-a)(e-a)(f-a)=\mu \mathrm{a}_{1} \mathrm{a}_{2} \mathrm{a}_{3} \mathrm{a}_{4}, \\
-\lambda(c-b)(d-b)(e-b)(f-b)=\mu \mathrm{b}_{1} \mathrm{~b}_{2} \mathrm{~b}_{3} \mathrm{~b}_{4} .
\end{array}
$$


We have thus

and

$$
\frac{(a-c)(b-c)}{(a-d)(b-d)}\left(\frac{c-k}{d-k}\right)^{2}=\frac{\mathrm{c}_{1} \mathrm{c}_{2} \mathrm{c}_{3} \mathrm{c}_{4}}{\mathrm{~d}_{1} \mathrm{~d}_{2} \mathrm{~d}_{3} \mathrm{~d}_{4}}
$$

$$
\frac{\mathrm{k}_{1}^{2}}{\mathrm{k}_{2}^{2}}=\frac{\mathrm{a}_{2} \mathrm{~b}_{2} \mathrm{c}_{1} \mathrm{~d}_{1} \mathrm{e}_{1} \mathrm{f}_{1}}{\mathrm{a}_{1} \mathrm{~b}_{1} \mathrm{c}_{2} \mathrm{~d}_{2} \mathrm{e}_{2} \mathrm{f}_{2}}
$$

which last equation, writing for a moment $\gamma, \delta=\sqrt{a_{2} b_{2} c_{1} d_{1} e_{1} f_{1}}, \sqrt{a_{1} b_{1} c_{2} d_{2} e_{2} f_{2}}$, gives $\frac{k-x_{1}}{k-x_{2}}=\frac{\gamma}{\delta}$, whence $k(\gamma-\delta)=x_{2} \gamma-x_{1} \delta$, and thence

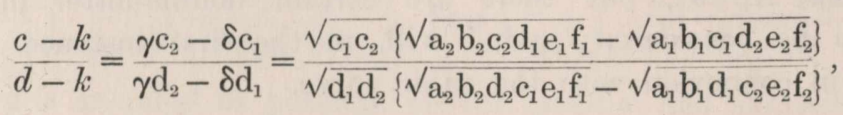

or, substituting in the first equation, we have

$$
\frac{\sqrt{(a-c)(b-c)}}{\sqrt{(a-d)(b-d)}} \cdot \frac{\sqrt{\mathrm{a}_{2} \mathrm{~b}_{2} \mathrm{c}_{2} \mathrm{~d}_{1} \mathrm{e}_{1} \mathrm{f}_{1}}-\sqrt{\mathrm{a}_{1} \mathrm{~b}_{1} \mathrm{c}_{1} \mathrm{~d}_{2} \mathrm{e}_{2} \mathrm{f}_{2}}}{\sqrt{\mathrm{a}_{2} \mathrm{~b}_{2} \mathrm{~d}_{2} \mathrm{c}_{1} \mathrm{e}_{1} \mathrm{f}_{1}}-\sqrt{\mathrm{a}_{1} \mathrm{~b}_{1} \mathrm{~d}_{1} \mathrm{c}_{2} \mathrm{e}_{2} \mathrm{f}_{2}}}=\frac{\sqrt{\mathrm{c}_{3} \mathrm{c}_{4}}}{\sqrt{\mathrm{d}_{3} \mathrm{~d}_{4}}} .
$$

159. Considering the duad $D E$ as an abbreviation for the double triad $A B C . D E F$, the expressed duad being always accompanied by the letter $F$, we are thus led to the consideration of the double-letter functions

$$
A B_{12}=\frac{1}{x_{1}-x_{2}}\left\{\sqrt{\mathrm{a}_{1} \mathrm{~b}_{1} \mathrm{f}_{1} \mathrm{c}_{2} \mathrm{~d}_{2} \mathrm{e}_{2}}-\sqrt{\mathrm{a}_{2} \mathrm{~b}_{2} \mathrm{f}_{2} \mathrm{c}_{1} \mathrm{~d}_{1} \mathrm{e}_{1}}\right\}, \text { \&c. }
$$

in connexion with the already mentioned single-letter functions $A_{12}=\sqrt{\mathrm{a}_{1} \mathrm{a}_{2}}$, \&c., viz. in this notation the equation just obtained is

$$
\frac{C_{34}}{D_{34}}=\sqrt{\frac{(a-c)(b-c)}{(a-d)(b-d)}} \frac{D E_{12}}{C E_{12}}
$$

and it thus appears that, the points 3, 4 being obtained as above from the given points 1, 2, then the quotient of two of the single-letter functions of 3,4 is a constant multiple of the quotient of two of tine double-letter functions of 1, 2. Observe that the points 3,4 are derived from 1,2 by means of the two points $a, b$ : we have $D E$ standing for $A B C . D E F, C E$ for $A B D . C E F$, and if the two functions were represented by $A B C, A B D$ respectively, then the form would have been

$$
\frac{C_{34}}{D_{34}}=\sqrt{\frac{(a-c)(b-c)}{(a-d)(b-d)}} \cdot \frac{A B C_{12}}{A B D_{12}},
$$

which is a clearer expression of the theorem; the apparent want of symmetry of the first form arises only from the arbitrary selection of the letter $F$ to accompany the expressed duad, and is at once removed by substituting for a duad $D E$ the triad $A B C . D E F$ which is thereby signified. The denominator factor $x_{1}-x_{2}$ is introduced in order to make the degree in $x_{1}$ or $x_{2}$ equal to that of the single-letter functions. 


\section{The Addition Theory. Art. Nos. 160 to 163.}

160. We have the six single-letter symbols $A, B, C, D, E, F$; viz. $A_{12}=\sqrt{\mathrm{a}_{1} \mathrm{a}_{2}}$, \&c. : and the ten double-letter symbols $A B, A C, A D, A E, B C, B D, B E, C D, C E, D E$, viz.

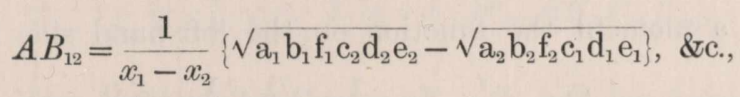

these 16 functions being connected by algebraical relations which are immediately deducible from these expressions of the functions in terms of $x_{1}, x_{2}$. The problem is to express the functions of 5,6 in terms of those of 1,2 and of those of 3,4. The relation between the variables $x_{1}, x_{2}, x_{3}, x_{4}, x_{5}, x_{6}$ consists herein that we have $x_{1}, x_{2}, x_{3}, x_{4}, x_{5}, x_{6}$ as the roots of the equation

$$
\left(\alpha x^{3}+\beta x^{2}+\gamma x+\delta\right)^{2}-\lambda(a-x)(b-x)(c-x)(d-x)(e-x)(f-x)=0 ;
$$

or, what is the same thing, it consists in the identity

$$
\begin{aligned}
\left(\alpha x^{3}+\beta x^{2}+\gamma x+\delta\right)^{2}-\lambda(a-x) & (b-x)(c-x)(d-x)(e-x)(f-x) \\
& -\mu\left(x_{1}-x\right)\left(x_{2}-x\right)\left(x_{3}-x\right)\left(x_{4}-x\right)\left(x_{5}-x\right)\left(x_{6}-x\right)=0 .
\end{aligned}
$$

Again, it may be expressed by the plexus of equations

$$
\left\|\begin{array}{cccccc}
1, & 1, & 1, & 1, & 1, & 1 \\
x_{1}, & x_{2}, & x_{3}, & x_{4}, & x_{5}, & x_{6} \\
x_{1}{ }^{2}, & x_{2}{ }^{2}, & x_{3}{ }^{2}, & x_{4}{ }^{2}, & x_{5}{ }^{2}, & x_{6}{ }^{2} \\
x_{1}{ }^{3}, & x_{2}{ }^{3}, & x_{3}{ }^{3}, & x_{4}{ }^{3}, & x_{5}{ }^{3}, & x_{6}{ }^{3} \\
\sqrt{ } X_{1}, & \sqrt{ } X_{2}, & \sqrt{ } X_{3}, & \sqrt{ } X_{4}, & \sqrt{ } X_{5}, & \sqrt{ } X_{6}
\end{array}\right\|=0
$$

where $X_{1}=\left(a-x_{1}\right)\left(b-x_{1}\right) \ldots\left(f-x_{1}\right)$, \&c.; these are equivalent of course to two equations, and serve to determine $x_{5}, x_{6}$ in terms of $x_{1}, x_{2}, x_{3}, x_{4}$.

161. The solution is, in fact, as is given in my paper "On the addition of the double 9-functions," Crelle, t. LXxxvIII. (1880), pp. 74-81, [703]. Writing successively $x=x_{1}, x_{2}, x_{3}, x_{4}$, we have

$$
\begin{aligned}
& \alpha x_{1}^{3}+\beta x_{1}^{2}+\gamma x_{1}+\delta=\sqrt{ } \lambda \sqrt{ } X_{1}, \\
& \alpha x_{2}^{3}+\beta x_{2}^{2}+\gamma x_{2}+\delta=\sqrt{ } \lambda \sqrt{ } X_{2}, \\
& \alpha x_{3}^{3}+\beta x_{3}{ }^{2}+\gamma x_{3}+\delta=\sqrt{ } \lambda \sqrt{ } X_{3}, \\
& \alpha x_{4}^{3}+\beta x_{4}{ }^{2}+\gamma x_{4}+\delta=\sqrt{ } \lambda \sqrt{ } X_{4},
\end{aligned}
$$

which equations serve to determine the ratios $\alpha: \beta: \gamma: \delta$ in terms of $x_{1}, x_{2}, x_{3}, x_{4}$; and we have then the two like equations

$$
\begin{aligned}
& \alpha x_{5}{ }^{3}+\beta x_{5}{ }^{2}+\gamma x_{5}+\delta=\sqrt{ } \lambda \sqrt{ } X_{5}, \\
& \alpha x_{6}{ }^{3}+\beta x_{6}{ }^{2}+\gamma x_{6}+\delta=\sqrt{ } \lambda \sqrt{ } X_{6},
\end{aligned}
$$

which determine the symmetric functions of $x_{5}, x_{6}$. 
If, reverting to the identity, we write therein for instance $x=a$, we find

$$
\alpha a^{3}+\beta a^{2}+\gamma \alpha+\delta=\sqrt{\mu} A_{12} A_{34} A_{56},
$$

which equation when properly reduced gives the proportional value of $A_{56}$.

162. Calling for a moment the function on the left-hand side $\Omega$, we have

$$
\left|\begin{array}{ccccc}
x_{1}^{3}, & x_{1}^{2}, & x_{1}, & 1, & \sqrt{ } \lambda \sqrt{ } X_{1} \\
x_{2}^{3}, & x_{2}^{2}, & x_{2}, & 1, & \sqrt{ } \lambda \sqrt{ } X_{2} \\
x_{3}^{3}, & x_{3}^{2}, & x_{3}, & 1, & \sqrt{ } \lambda \sqrt{ } X_{3} \\
x_{4}^{3}, & x_{4}^{2}, & x_{4}, & 1, & \sqrt{ } \lambda \sqrt{ } X_{4} \\
a^{3}, & a^{2}, & a, & 1, & \Omega
\end{array}\right|
$$

that is,

$$
\Omega\left|\begin{array}{cccc}
x_{1}{ }^{3}, & x_{1}{ }^{2}, & x_{1}, & 1 \\
x_{2}{ }^{3}, & x_{2}{ }^{2}, & x_{2}, & 1 \\
x_{3}{ }^{3}, & x_{3}{ }^{2}, & x_{3}, & 1 \\
x_{4}{ }^{3}, & x_{4}{ }^{2}, & x_{4}, & 1
\end{array}\right|+\sqrt{ } \lambda\left|\begin{array}{ccccc}
x_{1}^{3}, & x_{1}{ }^{2}, & x_{1}, & 1, & \sqrt{ } X_{1} \\
x_{2}{ }^{3}, & x_{2}{ }^{2}, & x_{2}, & 1, & \sqrt{ } X_{2} \\
x_{3}{ }^{3}, & x_{3}{ }^{2}, & x_{3}, & 1, & \sqrt{ } X_{3} \\
x_{4}^{3}, & x_{4}{ }^{2}, & x_{4}, & 1, & \sqrt{ } X_{4} \\
a^{3}, & a^{2}, & a, & 1, & 0
\end{array}\right|=0,
$$

viz. this is

$$
\begin{aligned}
& \Omega\left(x_{1}-x_{2}\right)\left(x_{1}-x_{3}\right)\left(x_{1}-x_{4}\right)\left(x_{2}-x_{3}\right)\left(x_{2}-x_{4}\right)\left(x_{3}-x_{4}\right) \\
&=-\sqrt{\lambda}\left\{\sqrt{X_{1}} \cdot x_{2}-x_{3} \cdot x_{2}-x_{4} \cdot x_{2}-a \cdot x_{3}-x_{4} \cdot x_{3}-a \cdot x_{4}-a\right. \\
&+\sqrt{X_{2}} \cdot x_{3}-x_{4} \cdot x_{3}-a \cdot x_{3}-x_{1} \cdot x_{4}-a \cdot x_{4}-x_{1} \cdot a-x_{1} \\
&+\sqrt{X_{3}} \cdot x_{4}-a \cdot x_{4}-x_{1} \cdot x_{4}-x_{2} \cdot a-x_{1} \cdot a-x_{2} \cdot x_{1}-x_{2} \\
&\left.+\sqrt{X_{4}} \cdot a-x_{1} \cdot a-x_{2} \cdot a-x_{3} \cdot x_{2}-x_{3} \cdot x_{2}-x_{4} \cdot x_{3}-x_{4}\right\},
\end{aligned}
$$

or, as this may be written,

$$
\begin{aligned}
\Omega \cdot x_{1}- & x_{3} \cdot x_{1}-x_{4} \cdot x_{2}-x_{3} \cdot x_{2}-x_{4} \\
= & \frac{\sqrt{\lambda} \cdot a-x_{3} \cdot a-x_{4}}{x_{1}-x_{2}}\left\{x_{2}-x_{3} \cdot x_{2}-x_{4} \cdot a-x_{2} \cdot \sqrt{X_{1}}-\left(x_{1}-x_{3} \cdot x_{1}-x_{4} \cdot a-x_{1} \cdot \sqrt{X_{2}}\right)\right\} \\
& +\frac{\sqrt{\lambda} \cdot a-x_{1} \cdot a-x_{2}}{x_{3}-x_{4}}\left\{x_{4}-x_{1} \cdot x_{4}-x_{2} \cdot a-x_{4} \cdot \sqrt{X_{3}}-\left(x_{3}-x_{1} \cdot x_{3}-x_{2} \cdot a-x_{3} \cdot \sqrt{X_{4}}\right)\right\} .
\end{aligned}
$$

We have here $\sqrt{\lambda}, a-x_{3}, a-x_{4}=\sqrt{\lambda} A^{2}{ }_{34}$, and the function

$$
\frac{1}{x_{1}-x_{2}}\left\{x_{2}-x_{3} \cdot x_{2}-x_{4} \cdot a_{2} \sqrt{X_{1}}-\left(x_{1}-x_{3} \cdot x_{1}-x_{4} \cdot a_{1} \sqrt{X_{2}}\right)\right\},
$$

which multiplies this, is without difficulty found to be

$$
=\frac{-A_{12}}{c-d \cdot d-b \cdot b-c} \Sigma\left\{c-d \cdot B_{34}^{2} B E_{12} \cdot C_{12} \cdot D_{12}\right\},
$$


where the summation extends to the three terms obtained by the cyclical interchange of the letters $b, c, d$ : these being a set of three out of the five letters other than $a$. Similarly $\sqrt{\lambda} \cdot a-x_{1} \cdot a-x_{2}$ is $=\sqrt{\lambda} A^{2}{ }_{12}$, and the function which multiplies this is

$$
=\frac{-A_{34}}{c-d \cdot d-b \cdot b-c} \Sigma\left\{c-d \cdot B_{12}^{2} \cdot B E_{34} C_{14} D_{14}\right\}
$$

The expression for $\Omega$ thus contains the factor $A_{12} A_{34}$ : but we have

$$
\Omega,=a \alpha^{3}+b \alpha^{2}+c \alpha+d,=\sqrt{\mu} A_{12} A_{34} A_{56} ;
$$

this equation contains therefore the factor $A_{12} A_{34}$, and omitting it we find

$$
\begin{aligned}
-\frac{\sqrt{\mu}}{\sqrt{\lambda}}\left(x_{1}-x_{3} \cdot x_{1}-x_{4} \cdot x_{2}-x_{3} \cdot x_{2}-x_{4}\right)(c-d . d-b . b-c) A_{56} \\
=A_{34} \Sigma\left\{c-d . B_{34}^{2} B E_{12} C_{12} D_{12}\right\}+A_{12} \Sigma\left\{c-d . B^{2}{ }_{12} B E_{34} C_{34} D_{14}\right\},
\end{aligned}
$$

where, as before, the summations refer each to the three terms obtained by the cyclical interchange of the letters $b, c, d$; these being any three of the five letters other than $a$ : and the remaining two letters $e, f$ enter into the formulæ symmetrically. The formula thus gives for $A_{56}$ ten values which are of course equal to each other.

By reason of the undetermined factor $\frac{\sqrt{\mu}}{\sqrt{\lambda}}$, the formula gives only the proportional value of $A_{56}$; viz. combining it with the like formulæ for $B_{56}$, \&c., we have determinate values of the ratios $A_{56}: B_{56}: \ldots: F_{56}$. But this being understood, we regard the formula as a formula for each single-letter function of $x_{5}, x_{6}$ in terms of the single and double-letter functions of $x_{1}, x_{2}$ and of $x_{3}, x_{4}$ respectively.

163. We require further the expressions for the double-letter functions of $x_{5}, x_{6}$. Consider for example the function $D E_{56}$, which is

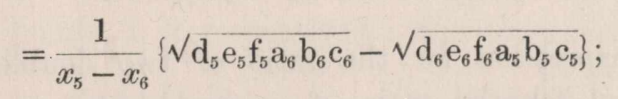

then multiplying by $A_{56} B_{56} C_{56},=\sqrt{\mathrm{a}_{5} \mathrm{~b}_{5} \mathrm{c}_{5} \mathrm{a}_{6} \mathrm{~b}_{6} \mathrm{c}_{6}}$, we have

$$
D E_{56} A_{56} B_{56} C_{56}=\frac{1}{x_{5}-x_{\mathrm{c}}}\left\{\mathrm{a}_{6} \mathrm{~b}_{6} \mathrm{c}_{6} \sqrt{X_{5}}-\mathrm{a}_{5} \mathrm{~b}_{5} \mathrm{c}_{5} \sqrt{X_{6}}\right\}
$$

or recollecting that $\sqrt{ } \lambda \sqrt{ } X_{5}$ and $\sqrt{ } \lambda \sqrt{ } X_{6}$ are $=\alpha x_{5}{ }^{3}+\beta x_{5}{ }^{2}+\gamma x_{5}+\delta$ and $\alpha x_{6}{ }^{3}+\beta x_{6}{ }^{2}+\gamma x_{6}+\delta$ respectively, this may be written

Using the well-known identity

$$
\begin{array}{r}
\sqrt{\lambda} D E_{56} A_{56} B_{56} C_{56}=\frac{1}{x_{5}-x_{6}}\left\{a-x_{6} . b-x_{6} . c-x_{6} .\left(\alpha x_{5}^{3}+\beta x_{5}^{2}+\gamma x_{5}+\delta\right)\right. \\
\left.-\left(a-x_{5} \cdot b-x_{5} \cdot c-x_{5}\right)\left(\alpha x_{e}{ }^{3}+\beta x_{6}{ }^{2}+\gamma x_{6}+\delta\right)\right\} .
\end{array}
$$

$$
\alpha x_{5}^{3}+\beta x_{5}{ }^{2}+\gamma x_{5}+\delta=\Sigma \cdot \alpha a^{3}+\beta a^{2}+\gamma a+\delta \cdot \frac{b-x_{5} \cdot c-x_{5} \cdot d-x_{5}}{b-a \cdot c-a \cdot d-a},
$$


where the summation extends to the four terms obtained by the cyclical interchanges of the letters $a, b, c, d$ : and the like identity for $\alpha x_{6}{ }^{3}+\beta x_{6}{ }^{2}+\gamma x_{6}+\delta$ : there will be terms in $\alpha a^{3}+\beta a^{2}+\gamma a+\delta, \quad \alpha b^{3}+\beta b^{2}+\gamma b+\delta, \quad \alpha c^{3}+\beta c^{2}+\gamma c+\delta$, but the term in $\alpha d^{3}+\beta d^{2}+\gamma d+\delta$ will disappear of itself. After some easy reductions, the result is

$$
\sqrt{\bar{\lambda}} D E_{56} A_{56} B_{56} C_{56}=\Sigma \frac{\alpha a^{3}+\beta a^{2}+\gamma \alpha+\delta}{b-a \cdot c-a} B_{56}^{2} C_{56}^{2}
$$

where the summation extends to the three terms obtained by the cyclical interchanges of the letters $a, b, c$. We have $\alpha a^{3}+\beta a^{2}+\gamma a+\delta=\sqrt{\mu} . A_{4_{2}} A_{34} A_{56}$, and similarly for the other two terms; the whole equation thus divides by $A_{56} B_{56} C_{56}$, and we find

$$
-\frac{\sqrt{\mu}}{\sqrt{\lambda}} D E_{56}=\frac{1}{b-c \cdot c-a \cdot a-b}\left(\frac{\sqrt{\mu}}{\sqrt{\lambda}}\right)^{2} \cdot \Sigma\left(b-c \cdot A_{12} A_{34} B_{56} C_{56}\right)
$$

in which equation, if we imagine $\frac{\sqrt{\mu}}{\sqrt{\lambda}} A_{56}, \frac{\sqrt{\mu}}{\sqrt{\lambda}} B_{56}, \frac{\sqrt{\mu}}{\sqrt{\lambda}} C_{56}$, each replaced by its value in terms of the single and double-letter functions of $x_{1}, x_{2}$ and $x_{3}, x_{4}$, we have an equation of the form

$$
-\frac{\sqrt{\mu}}{\sqrt{\lambda}}\left(x_{1}-x_{3} \cdot x_{1}-x_{4} \cdot x_{2}-x_{3} . x_{2}-x_{4}\right) D E_{56}=\frac{1}{x_{1}-x_{3} \cdot x_{1}-x_{4} \cdot x_{2}-x_{3} \cdot x_{2}-x_{4}} M
$$

where $M$ is a given rational and integral function of the single and double-letter functions of $x_{1}, x_{2}$ and $x_{3}, x_{4}$. The factor on the left-hand side has been made the same as in the formula for the single-letter functions $A_{56}$, \&c., and to do this it was necessary to bring in on the right-hand side the factor

$$
\frac{1}{x_{1}-x_{3} \cdot x_{1}-x_{4} \cdot x_{2}-x_{3} \cdot x_{2}-x_{4}}
$$

this disappears in the expression for the ratio of two double-letter functions; but it enters into the expression for the ratio of a single-letter to a double-letter function, and it then requires to be itself expressed in terms of the functions of $x_{1}, x_{2}$ and $x_{3}, x_{4}$ : it is easy to see that we have

$$
x_{1}-x_{3} . x_{1}-x_{4} \cdot x_{2}-x_{3} . x_{2}-x_{4}=\Sigma \frac{\left(A^{2}{ }_{34} B_{12}^{2}-A^{2}{ }_{12} B_{34}^{2}\right)\left(A^{2}{ }_{34} C^{2}{ }_{12}-A^{2}{ }_{12} C_{34}^{2}\right)}{(a-b)^{2}(a-c)^{2}},
$$

where the summation extends to the three terms obtained by the cyclical interchanges of the letters $a, b, c$ : these being a set of any three out of the six letters.

We have, in what precedes, obtained the expressions for the ratios of the 16 functions $A_{56}, \ldots, F_{56}, A B_{56}, \ldots, D E_{56}$ in terms of the ratios of the like functions of $x_{1}, x_{2}$ and $x_{3}, x_{4}$. 
Chapter VII. The Functions $T, U, V, \Theta$.

The present chapter is substantially a reproduction of C. and G.'s seventh section, "Die Function $T_{\xi \eta}$ ", borrowing only from the next section the definition of the theta-function; but for greater simplicity I consider for the most part, the case, fixed curve a quartic, $n=4, p=3$.

\section{Integral Form of the Affected Theorem. Art. Nos. 164 to 169.}

164. Writing for shortness $\frac{(x, y, z)_{12}{ }^{n-2} d \omega}{012}=d \Pi_{12}$, we are concerned with the integrals $\int_{a^{\prime}}^{a} d \Pi_{12}$ which present themselves in connexion with the affected theorem: the notation is explained, Chap. V.; $a, a^{\prime}$ are points on the curve, $f$; the variable may be any parameter serving for the determination of the current point, and the integral, taken from the value which belongs to the point $a^{\prime}$ to the value which belongs to the point $a$, is represented as above by means of the two points $a$, $a^{\prime}$ as limits of the integral. It is assumed that the integral is a canonical integral having the limits and the parametric points interchangeable, $\int_{a^{\prime}}^{a} d \Pi_{12}=\int_{2}^{1} d \Pi_{a a^{\prime}}$ : see Chapter IV.

165. Writing for shortness

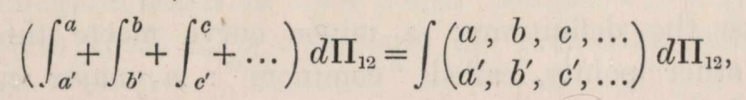

then if $\phi, \psi$ are curves each of the order $m$, the former of them intersecting the fixed curve $f$ in the points $a, b, c, \ldots$, and the latter of them intersecting the same curve in the points $a^{\prime}, b^{\prime}, c^{\prime}, \ldots$, and if $\phi_{1}, \psi_{1}, \phi_{2}, \psi_{2}$ are what the functions $\phi, \psi$ become on substituting therein in place of the current coordinates the values which belong to the parametric points 1, 2 respectively; the theorem becomes

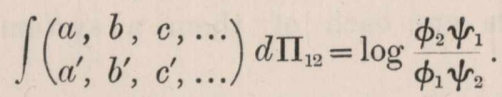

The superior limits may be interchanged in any manner, and so also the inferior limits may be interchanged in any manner. If a superior limit coincide with an inferior limit, the two may thus be considered as belonging to an integral which will then have the value 0 , and the coincident points may therefore be omitted from the expression on the left-hand side: and so in the case of any number of coincidences.

166. If the intersections of the curves $\phi, \psi$ and the parametric points are situate on a curve of the order $m$; then taking the equation of this curve to be $\phi+\lambda \psi=0$, we have simultaneously $\phi_{1}+\lambda \psi_{1}=0, \phi_{2}+\lambda \psi_{2}=0$; whence $\phi_{2} \psi_{1}=\psi_{1} \phi_{2}$, and the logarithmic term disappears: viz. the theorem becomes

$$
\int\left(\begin{array}{cc}
a, b, c, \ldots \\
a^{\prime}, b^{\prime}, & c^{\prime}, \ldots
\end{array}\right) d \Pi_{12}=0
$$

C. XII. 
167. Suppose that the curves $\phi, \psi$ are each of them a major curve, that is, a curve of the order $n-2$ passing through the $\delta \mathrm{dps}$, and consequently besides meeting the curve $f$ in $n(n-2)-2 \delta,=2 p+n-2$ points: the theorem is

$$
\int\left(\begin{array}{lll}
a, b, c, \ldots \\
a^{\prime}, & b^{\prime}, & c^{\prime}, \ldots
\end{array}\right) d \Pi_{12}=\log \frac{\phi_{2} \psi_{1}}{\phi_{1} \psi_{2}},
$$

where the numbers of the superior and of the inferior points are each $=2 p+n-2$.

168. Suppose further that the curves $\phi, \psi$, being major curves as above, pass each of them through the $n-2$ residues of 1,2 ; they besides meet in $(n-2)(n-3)$ points, viz. these are the $\delta \mathrm{dps}$ and $(n-2)(n-3)-\delta$ variable points: these $(n-2)(n-3)$ points lie on a minor curve, that is, a curve of the order $n-3$ passing through the dps; and the minor curve together with the parametric line 12 make together a major curve, passing through the intersections of $\phi, \psi$ and also through the parametric points 1,2: viz. these points and the intersections of $\phi, \psi$ are situate on a curve of the order $n-2$; the logarithmic term thus vanishes. The intersections of $\phi$ with the fixed curve are the $\delta \mathrm{dps}$, the $n-2$ residues and $2 p$ other points, say these are $a, b, c, \ldots, a^{\times}, b^{\times}, c^{\times}, \ldots ;$ similarly the curve $\psi$ meets the fixed curve in the $\delta \mathrm{dps}$, the $n-2$ residues, and in $2 p$ other points, say these are $d, e, f, \ldots, d^{\times}, e^{\times}, f^{\times}, \ldots$ : the theorem is

$$
\int\left(\begin{array}{l}
a, b, c, \ldots a^{\times}, b^{\times}, c^{\times}, \ldots \\
d, e, f, \ldots d^{\times}, e^{\times}, f^{\times}, \ldots
\end{array}\right) d \Pi_{12}=0,
$$

where there are $2 p$ superior and inferior points respectively.

169. I introduce the definitions: a minor curve meets the fixed curve in the dps and in $2 p-2$ other points, called "cominors": a major curve passing through the $n-2$ residues of the points 1,2 , meets the fixed curve in the $\delta$ dps, the $n-2$ residues and in $2 p$ other points, called "comajors in regard to the points 1, 2." Observe that $p-1$ of the cominors determine uniquely the remaining $p-1$ cominors; and similarly $p$ of the comajors determine uniquely the remaining $p$ comajors.

The foregoing theorem thus is that the sum $\int() d \Pi_{12}$ is $=0$, when the superior points and the inferior points are each of them a system of comajors in regard to the parametric points $1,2$.

Fixed Curve a Quartic. Art. No. 170.

170. It would be easy to go on with the general form; but as already mentioned, I prefer to consider the case, fixed curve a quartic, $n=4, p=3$. A minor curve is here a line meeting the quartic in 4 points, which are "cominors"; the major curve is a conic, and if this passes through the residues of 1,2 it besides meets the quartic in 6 points, which are "comajors in regard to the points 1, 2." Two points and their residues are cominors, but this is only by reason that $n-3=1$.

The Function T. Art. No. 171.

171. In conformity with C. and G., I introduce the functional symbol

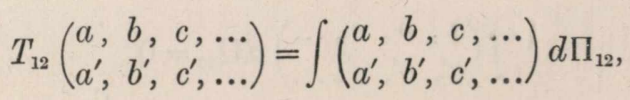


so that $T$ denotes a function of the parametric points, and of the sets of superior and inferior points respectively. The foregoing theorem for the quartic thus is

Observing that

$$
T_{12}\left(\begin{array}{lll}
a, b, c, & a^{\times}, b^{\times}, & c^{\times} \\
d, e, f, & d^{\times}, e^{\times}, f^{\times}
\end{array}\right)=0 .
$$

$$
\int_{d}^{a}+\int_{d \times}^{a \times}=2 \int_{d}^{a}-\int_{a \times}^{a}+\int_{d^{\times}}^{d}
$$

and so in other cases, this may be written

$$
2 T_{12}\left(\begin{array}{ll}
a, b, c \\
d, e, f
\end{array}\right)=T_{12}\left(\begin{array}{lll}
a, b, c & c \\
a^{\times}, b^{\times}, & c^{\times}
\end{array}\right)-T_{12}\left(\begin{array}{l}
d, e, f \\
d^{\times}, e^{\times}, f^{\times}
\end{array}\right) ;
$$

and if, as a definition of $T_{12}(a, b, c)$, we write

$$
T_{12}(a, b, c)=T_{12}\left(\begin{array}{lll}
a, & b, c \\
a^{\times}, & b^{\times}, & c^{\times}
\end{array}\right),
$$

where $a^{\times}, b^{\times}, c^{\times}$are the comajors of $a, b, c$ in regard to 1,2 , then the equation is

$$
2 T_{12}\left(\begin{array}{ll}
a, b, c \\
d, e, f
\end{array}\right)=T_{12}(a, b, c)-T_{12}(d, e, f),
$$

viz. the function of the $(2 p+2=) 8$ points $1,2, a, b, c, d, e, f$ is here expressed as a difference of two functions each of $(p+2=) 5$ points: $T_{12}(a, b, c)$ is regarded as a function of the 5 points $1,2, a, b, c$, because the remaining points $a^{\times}, b^{\times}, c^{\times}$depend only on these 5 points.

The Function U. Art. Nos. 172 to 175 .

172. We consider on the quartic the points $\xi, \mu ; 1,2,3$; and take $f, f^{\prime}$ for the cominors of 2,$3 ; g, g^{\prime}$ for the cominors of 3,1 ; and $h, h^{\prime}$ for the cominors of 1, 2. We write

$$
\begin{aligned}
& T=T_{\xi \mu}(1,2,3), \\
& T_{1}=T_{1 \mu}\left(\xi, f, f^{\prime}\right), \quad T_{2}=T_{2 \mu}\left(\xi, g, g^{\prime}\right), T_{3}=T_{3 \mu}\left(\xi, h, h^{\prime}\right)
\end{aligned}
$$

it is to be shown that there exists a function $U(1,2,3 ; \xi)$, such that

$$
\delta U=\frac{1}{2}\left\{\delta_{\xi} T+\delta_{1} T_{1}+\delta_{2} T_{2}+\delta_{3} T_{3}\right\}
$$

viz. considering $\xi, 1,2,3$ as variable points on the quartic, the whole infinitesimal variation of $U$ is the sum of these parts, where $\delta_{\xi} T$ is the variation of $T$ when only $\xi$ is varied, $\delta_{1} T_{1}$ the variation of $T_{1}$ when only 1 is varied, and similarly for $\delta_{2} T_{2}$ and $\delta_{3} T_{3}$. We consider in the proof three other points $4,5,6$ on the quartic; and taking $l, l^{\prime}$ for the cominors of 5,$6 ; m, m^{\prime}$ for those of 6,4 ; and $n, n^{\prime}$ for those of 4,5 , we write further

$$
X_{1}=T_{1 \mu}\left(4, l, l^{\prime}\right), \quad X_{2}=T_{2 \mu}\left(5, m, m^{\prime}\right), \quad X_{3}=T_{3 \mu}\left(6, n, n^{\prime}\right) ;
$$


and it then requires to be shown that

$$
\begin{aligned}
& \frac{1}{2} T \quad=\int\left(\begin{array}{l}
123 \\
456
\end{array}\right) d \Pi_{\xi \mu}+\frac{1}{2} T_{\xi \mu}(4,5,6), \\
& \frac{1}{2}\left(T_{1}-X_{1}\right)=\int\left(\begin{array}{l}
\xi 56 \\
423
\end{array}\right) d \Pi_{1 \mu}+\log \frac{\mu 23.156}{\mu 56.123}, \\
& \frac{1}{2}\left(T_{2}-X_{2}\right)=\int\left(\begin{array}{l}
\xi 64 \\
531
\end{array}\right) d \Pi_{2 \mu}+\log \frac{\mu 31.264}{\mu 64.123} \\
& \frac{1}{2}\left(T_{3}-X_{3}\right)=\int\left(\begin{array}{l}
\xi 45 \\
612
\end{array}\right) d \Pi_{3 \mu}+\log \frac{\mu 12.345}{\mu 45.123}
\end{aligned}
$$

where $\mu 23$ is the determinant formed with the coordinates of the points $\mu, 2,3$ respectively: and so in other cases.

173. We have

that is,

$$
T_{\xi \mu}\left(\begin{array}{ll}
1, & 2,3 \\
4, & 5,6
\end{array}\right)=\frac{1}{2} T_{\xi \mu}(1,2,3)-\frac{1}{2} T_{\xi \mu}(4,5,6)
$$

and thence the above value of $\frac{1}{2} T$.

$$
=\frac{1}{2} T \quad-\frac{1}{2} T_{\xi \mu}(4,5,6)
$$

The affected theorem gives

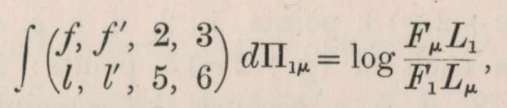

where $F=0$ is the equation of the line through $f, f^{\prime}, 2,3$; and $F_{1}, F_{\mu}$ are what the function $F$ becomes, on substituting therein for the current coordinates the coordinates of the points $1, \mu$ respectively. And similarly $L=0$ is the equation of the line through $l, l^{\prime}, 5,6$; and $L_{1}, L_{\mu}$ are what the function $L$ becomes by the same substitutions respectively. The values of $F_{1}, F_{\mu}$ are $123, \mu 23$ : those of $L_{1}, L_{\mu}$ are 156 , $\mu 56$; and the logarithmic term is thus

We then have

$$
=\log \frac{\mu 23.156}{\mu 56.123} \text {. }
$$

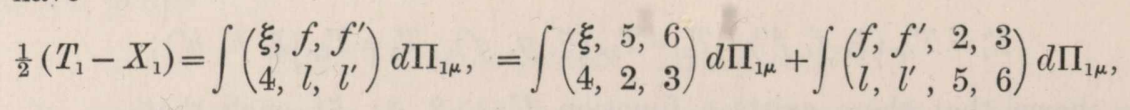

and in this last expression for $\frac{1}{2}\left(T_{1}-X_{1}\right)$ substituting for the second term the logarithmic value just obtained, we have the required expression for $\frac{1}{2}\left(T_{1}-X_{1}\right)$ : and those for $\frac{1}{2}\left(T_{2}-X_{2}\right)$ and $\frac{1}{2}\left(T_{3}-X_{3}\right)$ are deduced by mere cyclical permutations of the letters.

174. Returning to the assumed relation $\delta U=\frac{1}{2}\left\{\delta_{\xi} T+\delta_{1} T_{1}+\delta_{2} T_{2}+\delta_{3} T_{3}\right\}$; in order to the existence of the function $U$, it is only necessary to show that $T-T_{1}$ contains no term in $1, \xi$, that is, no term depending on both these points, and that $T_{1}-T_{2}$ contains no term in 1,2 : for then, by symmetry, the like properties hold in regard to $T-T_{2}, T-T_{3}, T_{1}-T_{3}, T_{2}-T_{3}$ respectively, and the assumed expression is a complete differential, from which the function $U$ may be obtained by integration. 
175. To show that $T-T_{1}$ contains no term in $1, \xi$.

For $T$, the only term in $1, \xi$ is $\int_{4}^{1} d \Pi_{\xi \mu}$,

$$
\text { " } T_{1} \quad, \quad \quad \quad \int_{4}^{\xi} d \Pi_{1 \mu}
$$

and it is to be shown that the difference of the two integrals contains no term in $1, \xi$. Considering on the quartic the two new points $\delta, \epsilon$, the first integral is

$$
\int\left(\begin{array}{ll}
1, & \delta \\
\delta, & 4
\end{array}\right)\left(d \Pi_{\xi \epsilon}+d \Pi_{\epsilon \mu}\right),=\int_{\delta}^{1} d \Pi_{\xi \epsilon}+\int_{4}^{\delta} d \Pi_{\xi \epsilon}+\int_{4}^{1} d \Pi_{\epsilon \mu},
$$

and the second is

$$
\int\left(\begin{array}{ll}
\xi, & \epsilon \\
\epsilon, 4
\end{array}\right)\left(d \Pi_{1 \delta}+d \Pi_{\delta \mu}\right),=\int_{\epsilon}^{\xi} d \Pi_{1 \delta}+\int_{4}^{\epsilon} d \Pi_{\delta \mu}+\int_{\mu}^{\xi} d \Pi_{\delta \mu} .
$$

Hence in the difference the only terms which can contain $1, \xi$ are

$$
\int_{\delta}^{1} d \Pi_{\xi \epsilon}-\int_{\epsilon}^{\xi} d \Pi_{1 \delta}
$$

and this is $=0$ : wherefore there is not in the difference any term in $1, \xi$. This proves the property for $T-T_{1}$. The property for $T_{1}-T_{2}$ is proved in a similar manner.

Theorems in regard to the Function U. Art. Nos. 176 to 179.

176. Theorem (A). To prove

we have

$$
U(1,2,3 ; \xi)-U(1,2,3 ; \mu)=\frac{1}{2} T_{\xi \mu}(1,2,3),
$$

$$
\begin{aligned}
U(1,2,3 ; \xi)-U(1,2,3 ; \mu) & =\int_{\mu}^{\xi} d_{\xi} U=\frac{1}{2} \int_{\mu}^{\xi} d_{\xi} T \\
& =\frac{1}{2} T_{\xi \mu}(1,2,3)-\frac{1}{2} T_{\mu \mu}(1,2,3),
\end{aligned}
$$

and $T_{\mu \mu}(1,2,3)=\int\left(\begin{array}{lll}1, & 2 & 3 \\ 1^{\times} & 2^{\times} & 3^{\times}\end{array}\right) d \Pi_{\mu \mu}$, where $d \Pi_{\mu \mu}=0$, viz. considering this as derived from $d \Pi_{\xi \mu},=Q_{\xi \mu} d \omega$, by making the point $\xi$ coincide with $\mu$, then when $\xi$ is indefinitely near to $\mu$, the numerator and denominator of $Q_{\xi \mu}$ are each of them infinitesimal of the orders 3 and 2 respectively, and thus the function $Q_{\xi \mu}$ ultimately vanishes (see as to this, Chap. V. Art. Nos. 99 to 106). We have therefore $T_{\mu \mu}(1,2,3)=0$, and the required theorem is proved.

177. Theorem (B). To prove

$$
U(1,2,3 ; \xi)-U(4,2,3 ; \xi)=\frac{1}{2} T_{14}\left(\xi, f, f^{\prime}\right),
$$

where, as before, $f, f^{\prime}$ are the cominors of 2,3 , that is, $2,3, f, f^{\prime}$ lie on a line, we have

$$
\begin{aligned}
U(1,2,3 ; \xi)-U(4,2,3 ; \xi) & =\int_{4}^{1} d_{1} U=\frac{1}{2} \int_{4}^{1} d_{1} T_{1} \\
& =\frac{1}{2} T_{1 \mu}\left(\xi, f, f^{\prime}\right)-\frac{1}{2} T_{4 \mu}\left(\xi, f, f^{\prime}\right)
\end{aligned}
$$


the point $\mu$ is arbitrary, and it may be taken to coincide with 4 ; but we then have $T_{44}\left(\xi, f, f^{\prime}\right)=0$, and the theorem is thus proved.

178. Theorem (C). We have

$$
T_{11 \times}\left(\xi, f, f^{\prime}\right)+T_{11 \times}\left(\eta, k, k^{\prime}\right)=0 ;
$$

where $\xi, \eta, 1,2,3$ are arbitrary points on the quartic; $1^{\times}, 2^{\times}, 3^{\times}$are the comajors of $1,2,3$ in regard to $\xi, \eta$, viz. the points $1,2,3,1^{\times}, 2^{\times}, 3^{\times}$lie on a conic which passes through $\xi^{\prime}, \eta^{\prime}$ the residues (or cominors) of $\xi, \eta: f, f^{\prime}$ are the cominors of 2,3 ; and $k, k^{\prime}$ are the cominors of $2^{\times}, 3^{\times}$.

Taking $\theta, \theta^{\prime}$ for the cominors of $1,1^{\times}$, the four lines $\xi \eta \xi^{\prime} \eta^{\prime}, 11^{\times} \theta \theta^{\prime}, 23 f f^{\prime}$ and $2^{\times} 3^{\times} k k^{\prime}$ form a quartic cutting the fixed quartic in the 16 points: but of these, $\xi^{\prime}, \eta^{\prime}, 1,2,3,1^{\times}, 2^{\times}, 3^{\times}$lie in a conic: hence the remaining 8 points $\theta, \theta^{\prime}, \xi, \eta$, $f, f^{\prime}, k, k^{\prime}$ lie in a conic; that is, $\xi, \eta, f, f^{\prime}, k, k^{\prime}$ lie on a conic through $\theta, \theta^{\prime}$, the residues of $1,1^{\times}$, or they are comajors in regard to $1,1^{\times}$; whence the theorem.

179. We have

$$
\begin{aligned}
& \frac{1}{2} T_{11 \times}\left(\xi, f, f^{\prime}\right)=U\left(\xi, f, f^{\prime} ; 1\right)-U\left(\xi, f, f^{\prime} ; 1^{\times}\right),=U(1,2,3 ; \xi)-U\left(1^{\times}, 2,3 ; \xi\right), \\
& \frac{1}{2} T_{11 \times}\left(\eta, k, k^{\prime}\right)=U\left(\eta, k, k^{\prime} ; 1\right)-U\left(\eta, k, k^{\prime} ; 1^{\times}\right),=U\left(1,2^{\times}, 3^{\times} ; \eta\right)-U\left(1^{\times}, 2,3 ; \eta\right) ;
\end{aligned}
$$

viz. we have thus two expressions for each term of the equation (C),

$$
T_{11 \times}\left(\xi, f, f^{\prime}\right)+T_{11 \times}\left(\eta, k, k^{\prime}\right)=0 .
$$

In particular, we have Theorem (D)

$$
U(1,2,3 ; \xi)-U\left(1^{\times}, 2,3 ; \xi\right)=-U\left(1,2^{\times}, 3^{\times} ; \eta\right)+U\left(1^{\times}, 2^{\times}, 3^{\times} ; \eta\right) .
$$

Again, we have $T_{\xi \eta}(1,2,3)+T_{\xi \eta}\left(1^{\times}, 2^{\times}, 3^{\times}\right)=0 ;$ where $1,2,3,1^{\times}, 2^{\times}, 3^{\times}$are comajors in regard to $\xi, \eta$ : and

$$
\begin{aligned}
& \frac{1}{2} T_{\xi \eta}(1,2,3)=U(1,2,3 ; \xi)-U(1,2,3 ; \eta), \\
& \frac{1}{2} T_{\xi \eta}\left(1^{\times}, 2^{\times}, 3^{\times}\right)=U\left(1^{\times}, 2^{\times}, 3^{\times}, \xi\right)-U\left(1^{\times}, 2^{\times}, 3^{\times}, \eta\right) ;
\end{aligned}
$$

whence Theorem (E),

$$
U(1,2,3 ; \xi)-U(1,2,3 ; \eta)=-U\left(1^{\times}, 2^{\times}, 3^{\times} ; \xi\right)+U\left(1^{\times}, 2^{\times}, 3^{\times}, \eta\right) .
$$

\section{The Function V. Art. Nos. 180 to 182.}

180. It is convenient to consider $U$ as a logarithm, say

$$
-U(1,2,3 ; \xi)=\log V(1,2,3 ; \xi) \text {, or } V(1,2,3 ; \xi)=\exp -U(1,2,3 ; \xi) \text {; }
$$

$V$, like $U$, is a function of the $(p+1=) 4$ points $1,2,3, \xi$, on the quartic.

The equation (D) thus becomes

$$
\frac{V(1,2,3 ; \xi)}{V\left(1^{\times}, 2^{\times}, 3^{\times} ; \eta\right)}=\frac{V\left(1^{\times}, 2,3 ; \xi\right)}{V\left(1,2^{\times}, 3^{\times} ; \eta\right)},
$$


where $1,2,3,1^{\times}, 2^{\times}, 3^{\times}$are comajors in regard to $\xi, \eta$ : the equation shows that, in the function $\frac{V(1,2,3 ; \xi)}{V\left(1^{\times}, 2^{x}, 3^{\times} ; \eta\right)}$, we can without alteration of the value interchange a pair of points $1,1^{\times}$out of the system of comajor points; and it of course follows that we can in any manner whatever interchange these points, so as to have any three of them in the numerator-function and the remaining three in the denominatorfunction. In particular, we have

$$
\frac{V(1,2,3 ; \xi)}{V\left(1^{\times}, 2^{\times}, 3^{\times} ; \eta\right)}=\frac{V\left(1^{\times}, 2^{\times}, 3^{\times} ; \xi\right)}{V(1,2,3 ; \eta)} .
$$

The equation (E) becomes

and multiplying we find

$$
\frac{V(1,2,3 ; \xi)}{V(1,2,3 ; \eta)}=\frac{V\left(1^{\times}, 2^{\times}, 3^{\times} ; \eta\right)}{V\left(1^{\times}, 2^{\times}, 3^{\times} ; \xi\right)}
$$

$$
V^{2}(1,2,3 ; \xi)=V^{2}\left(1^{\times}, 2^{\times}, 3^{\times} ; \eta\right)
$$

that is, $V(1,2,3 ; \xi)= \pm V\left(1^{\times}, 2^{\times}, 3^{\times} ; \eta\right)$, the sign being determinately + or determinately -, according to the precise definition of the function $V$.

181. Considering $\eta$ and also $1^{\times}, 2^{\times}, 3^{\times}$as fixed points on the curve; but $\xi$ as a variable point (that is, the parametric line $\xi \eta$ as rotating about the fixed point $\eta$ ), the points 1, 2, 3 are then determined as the remaining intersections with the quartic of the conic which passes through the points $1^{\times}, 2^{\times}, 3^{\times}$and through the points $\xi^{\prime}, \eta^{\prime}$, which are the residues of $\xi, \eta$. And by the theorem just obtained it appears that, $\xi, 1,2,3$ thus varying, the function $V(1,2,3 ; \xi)$ remains constant. This comes to saying that $V$, considered as a function of the points $1,2,3, \xi$, satisfies a certain linear partial differential equation of the first order, having a solution $V=F(u, v, w)$, an arbitrary function of $u, v, w$, determinate functions of the points 1, 2, 3, $\xi$. And if we can find $u, v, w$ functions of these points such that they each of them remain constant when the points $1,2,3, \xi$ vary as above, then the arbitrary function of $u, v, w$ will remain constant for the variation in question and will thus be a value of the function $V$.

182. It is easily seen that such functions are

$$
u, v, w=\left(\int^{1}+\int^{2}+\int^{3}-\int^{\xi}\right) x d \omega, y d \omega, z d \omega
$$

the inferior limits being given points which are regarded as absolute constants. For by the pure theorem, we have

$$
\Sigma(x, y, z)^{1} d \omega=0,
$$

where $(x, y, z)^{1}$ is an arbitrary linear function, and where the summation extends to all the intersections of the quartic with any given curve. Writing

$$
p=\int x d \omega, \int y d \omega \text { or } \int z d \omega, \text { that is, } p_{1}=\int^{1} x d \omega, \int^{1} y d \omega \text { or } \int^{1} z d \omega,
$$

the inferior limits being any absolutely fixed point on the curve, and similarly $p_{2}$, \&c.; the integral form of the theorem is $\Sigma p=$ constant. And applying the theorem 
successively to the parametric line, and to the conic which determines the points $1,2,3$, we have

$$
\begin{aligned}
& p_{\xi}+p_{\eta}+p_{\xi^{\prime}}+p_{\eta^{\prime}}=\text { const. } \\
& p_{\xi^{\prime}}+p_{\eta^{\prime}}+p_{1}+p_{2}+p_{3}+p_{1} \times+p_{2} \times+p_{3} \times=\text { const. }
\end{aligned}
$$

Taking the difference of these equations, we have

$$
p_{1}+p_{2}+p_{3}-p_{\xi}+p_{1} \times+p_{2} \times+p_{3} \times-p_{\eta}=\text { const. }
$$

viz. the points $\eta, 1^{\times}, 2^{\times}, 3^{\times}$being fixed points, this is

$$
p_{1}+p_{2}+p_{3}-p_{\xi}=\text { const., }
$$

that is, the functions $u, v, w$ defined as above are each of them constant under the variation in question.

The Function $\Theta$. Art. Nos. 183 and 184.

183. The function $V(1,2,3 ; \xi)$ of the $(p+1=) 4$ points $1,2,3, \xi$, is thus a function of the $(p=) 3$ arguments

$$
u, v, w,=\left(\int^{1}+\int^{2}+\int^{3}-\int^{\xi}\right) x d \omega, y d \omega, z d \omega .
$$

Disregarding a constant and exponential factor, we say that it is a theta-function of these arguments, and we write the result provisionally in the form

$$
V(1,2,3 ; \xi)=\Theta(u, v, w)
$$

the more precise definition of the theta-function being reserved for further consideration.

184. It appears by what precedes that a sum of $(p=) 3$ integrals $\int\left(\begin{array}{l}a b c \\ d e f\end{array}\right) d \Pi_{12}$, otherwise called $T_{12}\left(\begin{array}{l}a, b, c \\ d, e, f\end{array}\right)$, is in the first place expressed (see No. 171) as a difference, $=\frac{1}{2} T_{12}(a, b, c)-\frac{1}{2} T_{12}(d, e, f)$, of two functions $T$. Each of these is by Theorem (A) (No. 176) expressed as a difference of two functions $U$, that is, as the difference of the logarithms, or logarithm of the quotient, of two functions $V$ : such function $V$ is according to its original definition a function of $(p+1=) 4$ points, but in such wise that the function is expressible as a function of $(p=) 3$ arguments, and so expressed it is a $\Theta$-function of these arguments: and the final result thus is that the sum of $(p=) 3$ integrals $\int\left(\begin{array}{ll}a, b, c \\ d, e, f\end{array}\right) d \Pi_{12}$ is equal to the logarithm of a fraction, whereof the numerator and the denominator are each of them a product of two $\Theta$-functions. 